University of Louisville

ThinkIR: The University of Louisville's Institutional Repository

Electronic Theses and Dissertations

8-2012

\title{
CAD system for lung nodule analysis.
}

\author{
Cambron N. Carter
}

University of Louisville

Follow this and additional works at: https://ir.library.louisville.edu/etd

\section{Recommended Citation}

Carter, Cambron N., "CAD system for lung nodule analysis." (2012). Electronic Theses and Dissertations. Paper 218.

https://doi.org/10.18297/etd/218

This Master's Thesis is brought to you for free and open access by ThinkIR: The University of Louisville's Institutional Repository. It has been accepted for inclusion in Electronic Theses and Dissertations by an authorized administrator of ThinkIR: The University of Louisville's Institutional Repository. This title appears here courtesy of the author, who has retained all other copyrights. For more information, please contact thinkir@louisville.edu. 


\title{
CAD SYSTEM FOR LUNG NODULE ANALYSIS
}

By

Cambron N. Carter

B.S., University of Louisville, 2010

B.S., University of Louisville, 2011

\author{
A Thesis \\ Submitted to the Faculty of the \\ University of Louisville \\ J. B. Speed School of Engineering \\ as Partial Fulfillment of the Requirements \\ for the Professional Degree
}

\section{MASTER OF ENGINEERING}

Department of Electrical and Computer Engineering

August 2012 


\section{CAD SYSTEM FOR LUNG NODULE ANALYSIS}

Submitted by:

Cambron Carter

A Thesis Approved on

(Date)

by the Following Reading and Examination Committee:

Dr. Aly A. Farag, Thesis Director

Dr. James Graham

Dr. Thomas Starr

Dr. Robert Falk 


\section{ACKNOWLEDGEMENTS}

I wish to thank my mother, Brenda Carter, my second mother Linda Smith, my father Boyce Carter, my three brothers Bryan, Chad, and Scott and the rest of my family for their ongoing support throughout this process. Further, I would like to thank Rosario Pusateri for being nothing short of a brother during our time in the CVIP lab. I would like to thank Dr. Aly Farag for the opportunity to conduct research, Dr. Donqing Chen, Dr. Ham Rara, Mr. Mike Miller, Dr. Melih Aslan, Mrs. Shireen Elhabien, Dr. Hossam Abdelmunim, Dr. Amal Farag, and all other members of the CVIP Lab both past and present that I have shared this experience with. Lastly, I would like to thank my committee members, Dr. James Graham, Dr. Thomas Starr and Dr. Robert Falk for their time and patience during my education. 


\begin{abstract}
Lung cancer is the deadliest type of known cancer in the United States, claiming hundreds of thousands of lives each year. However, despite the high mortality rate, the 5year survival rate after resection of Stage 1A non-small cell lung cancer is currently in the range of $62 \%-82 \%$ and in recent studies even $90 \%$. Patient survival is highly correlated with early detection. Computed Tomography (CT) technology services the early detection of lung cancer tremendously by offering a minimally invasive medical diagnostic tool. Some early types of lung cancer begin with a small mass of tissue within the lung, less than $3 \mathrm{~cm}$ in diameter, called a nodule. Most nodules found in a lung are benign, but a small population of them becomes malignant over time. Expert analysis of CT scans is the first step in determining whether a nodule presents a possibility for malignancy but, due to such low spatial support, many potentially harmful nodules go undetected until other symptoms motivate a more thorough search.
\end{abstract}

Computer Vision and Pattern Recognition techniques can play a significant role in aiding the process of detecting and diagnosing lung nodules. This thesis outlines the development of a CAD system which, given an input CT scan, provides a functional and fast, second-opinion diagnosis to physicians. The entire process of lung nodule screening has been cast as a system, which can be enhanced by modern computing technology, with the hopes of providing a feasible diagnostic tool for clinical use. It should be noted that the proposed CAD system is presented as a tool for experts - not a replacement for them. The primary motivation of this thesis is the design of a system that could act as a catalyst for reducing the mortality rate associated with lung cancer. 


\section{Table of Contents}

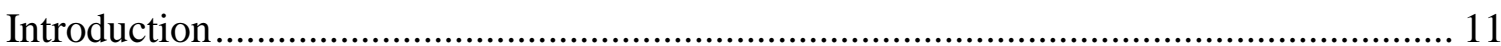

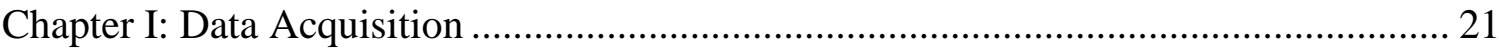

1.1 Currently Available Lung Nodule Data ........................................................... 21

1.2 Data Acquisition in CAD System ………………….................................... 25

Chapter 2: Tissue Segmentation ................................................................................. 30

2.1 Overview and Motivations ………………………................................... 30

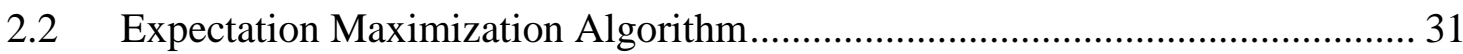

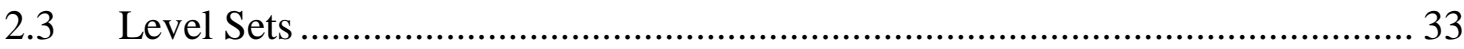

Chapter 3: Nodule Detection ...................................................................................... 40

3.1 Overview, Motivations and Challenges …………...................................... 40

3.2 Nodule Modeling and Detection ....................................................................... 41

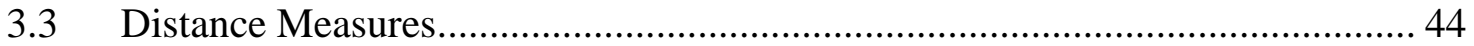

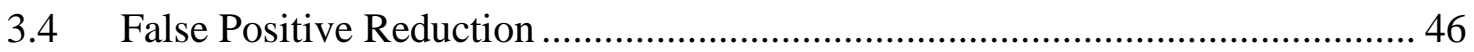

3.5 Tensor Modeling for Malignancy Classification ............................................... 49

3.5.1 Building a Data Tensor with Lung Nodules .............................................. 50

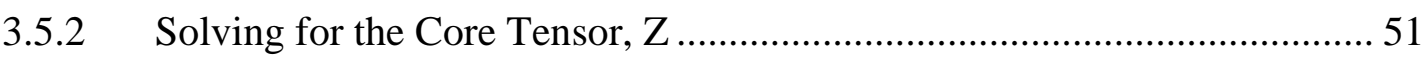

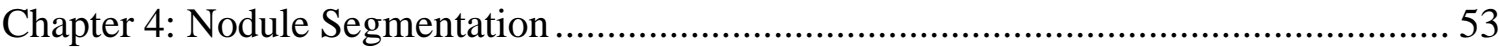

4.1 Overview, Motivations and Challenges ........................................................... 53

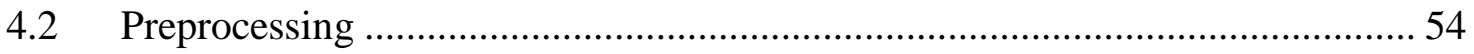

4.3 Level Sets with Shape Priors........................................................................ 55

4.4 Expectation Maximization Algorithm for Nodule Segmentation ...................... 57

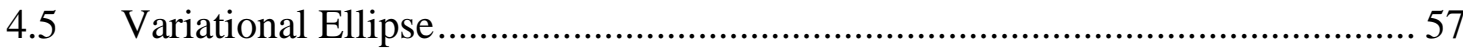

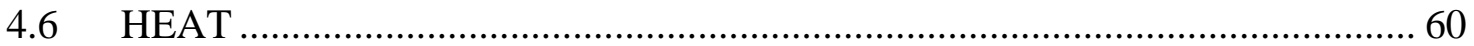

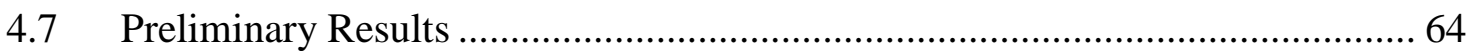

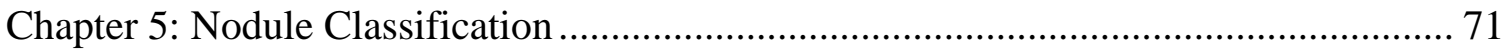

5.1 Overview, Motivations and Challenges ............................................................... 71

5.2 Preliminary Feature Descriptors and Classifiers ............................................... 76

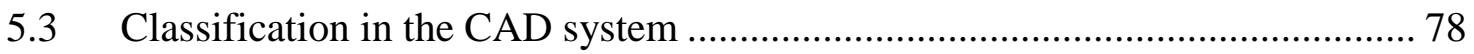

Chapter 6: A Functional User Interface ........................................................................ 80 


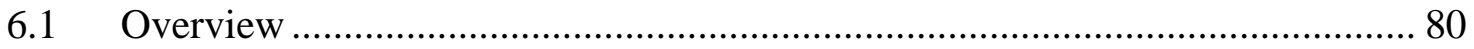

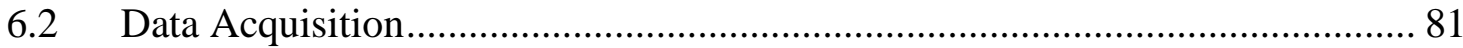

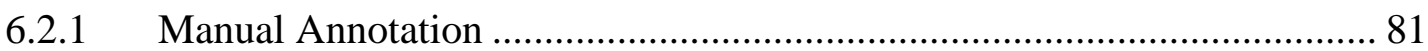

6.2.2 Enrolling a Nodule in Modeling Dataset .................................................. 86

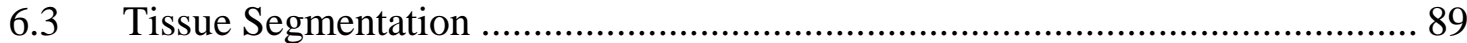

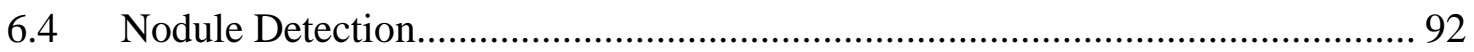

6.5 Nodule Segmentation and Classification ....................................................... 98

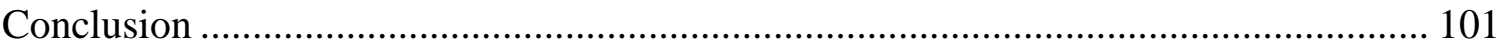

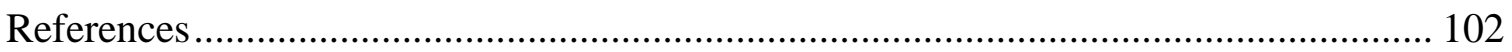

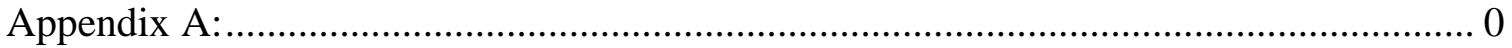




\section{List of Tables}

Table 1: Publicly available Lung Nodule Databases ................................................... 22

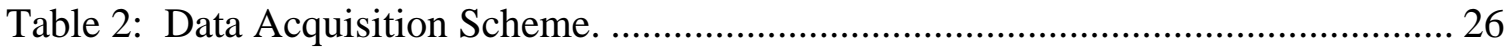

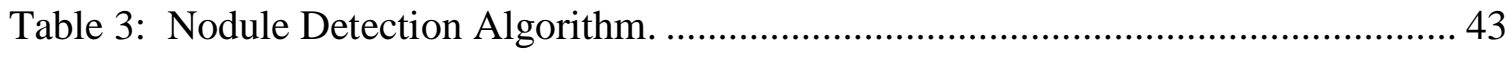

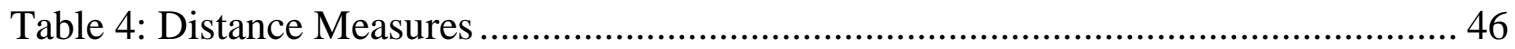

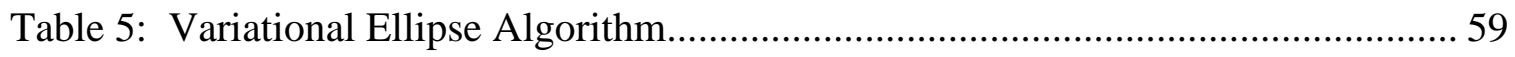

Table 6: Hosseini et. al Classification Features ............................................................. 72

Table 7: Classification Scheme implemented in the CAD system ................................ 79

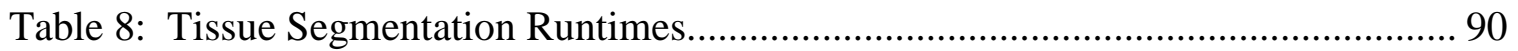

Table 9: Average Runtimes per Slice for Nodule Detection. ........................................ 98

Table 10: Nodule Segmentation Runtimes ................................................................ 99

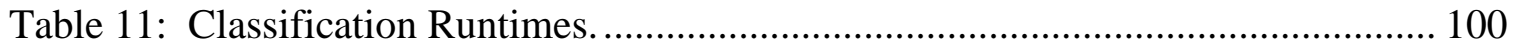




\section{List of Figures}

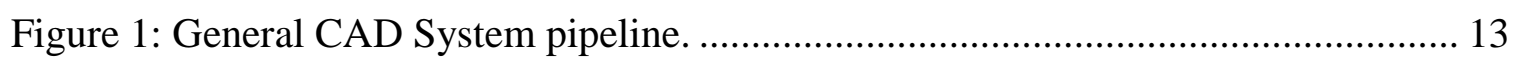

Figure 2: Four lung nodule types used in the proposed system..................................... 14

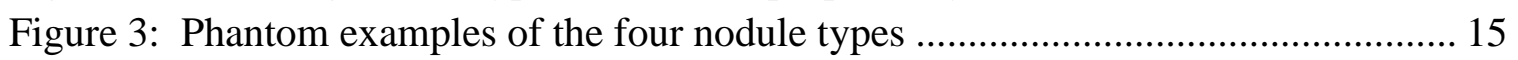

Figure 4: Kostis' framework for lung nodule classification........................................... 16

Figure 5: A more detailed outline of the CAD System................................................ 17

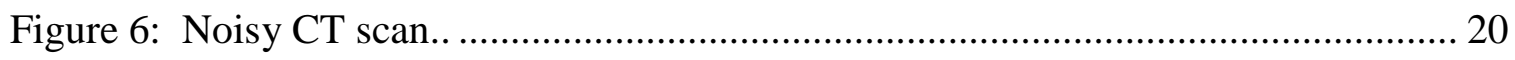

Figure 7: Sample of expert nodule outlines provided by 4 radiologists ........................... 24

Figure 8: Synthetic examples of contours corresponding to nodule morphology …….... 27

Figure 9: Synthetic examples of benign calcification patterns ..................................... 28

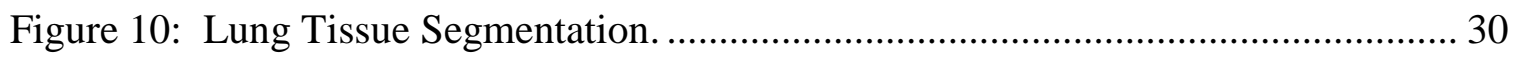

Figure 11: Signed Distance Maps of the four nodule types............................................ 33

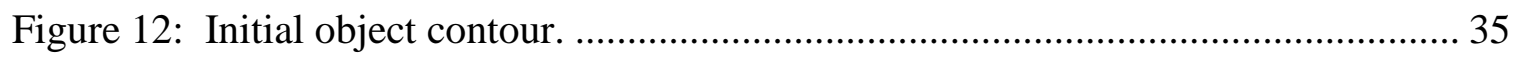

Figure 13: Binary edge map of the initial object region ............................................. 36

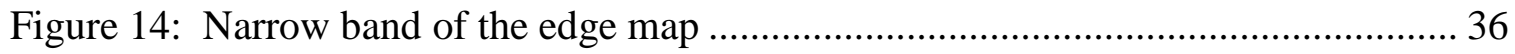

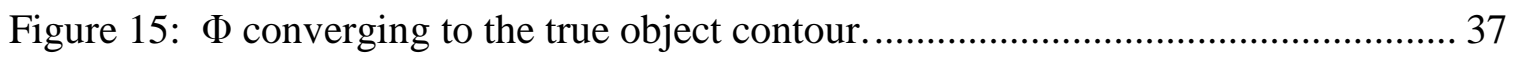

Figure 16: Output of statistical level sets algorithm.................................................... 38

Figure 17: 2-D projections obtained from 3-D Statistical Level-Sets algorithm............. 39

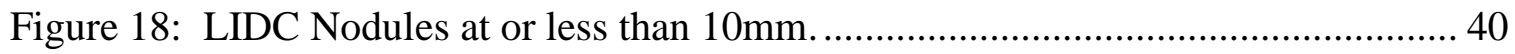

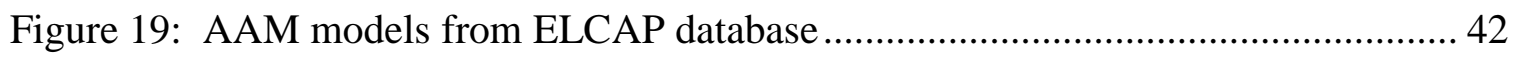

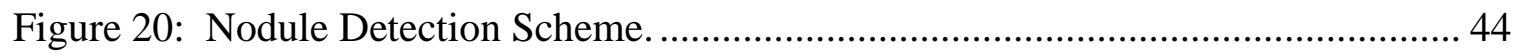

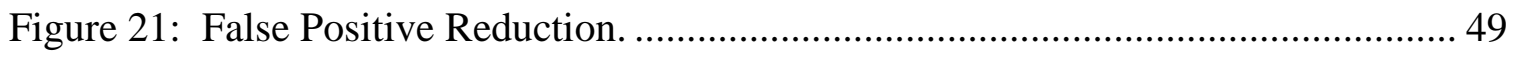

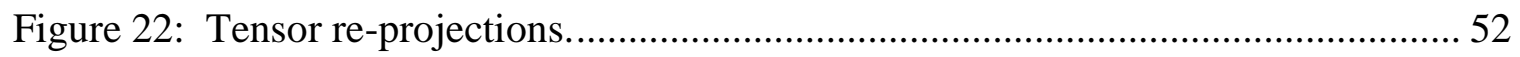

Figure 23: Level set algorithm as applied to nodule segmentation.. …………………... 56

Figure 24: Sample results from the level set method driven with shape priors ............... 57

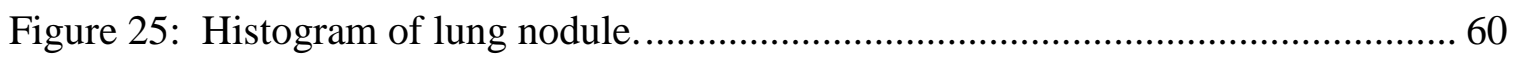

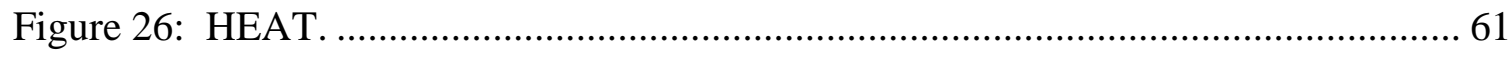

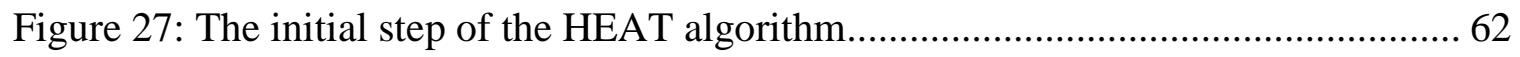

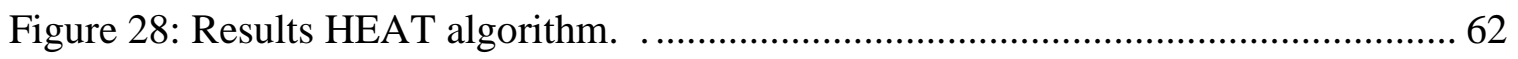

Figure 29: 3D nodule reconstruction obtained from HEAT. .............................................. 63

Figure 30: Comparison of LIDC nodule segmentation results.......................................... 64

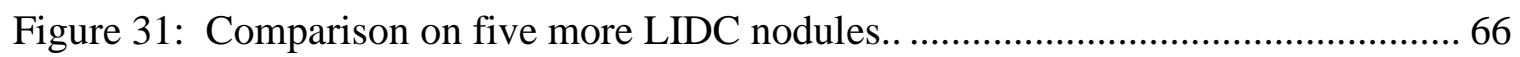

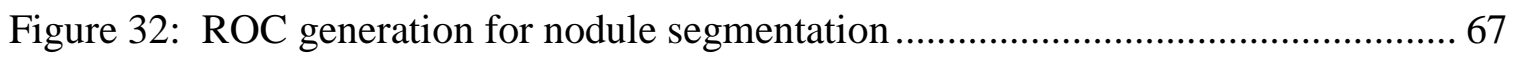

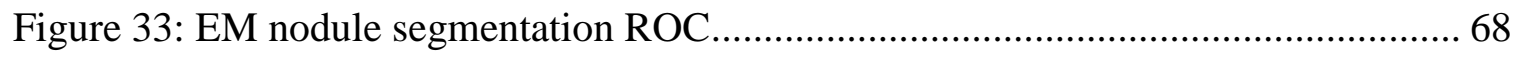

Figure 34: Variational Ellipse + EM nodule segmentation ROC. ………………..............6 69

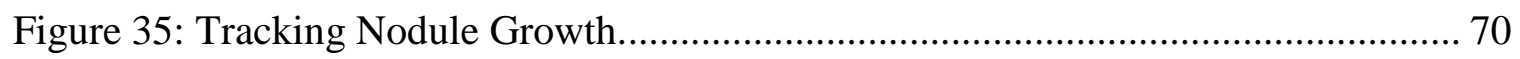

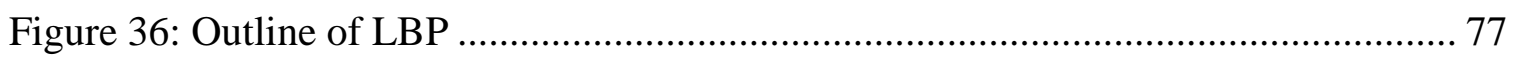

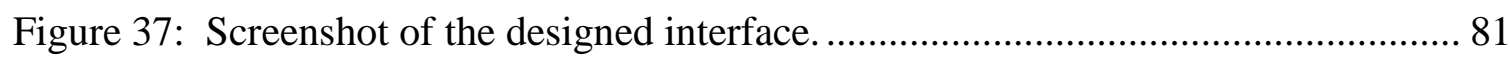

Figure 38: Prompting the user to Load. ..................................................................... 82 


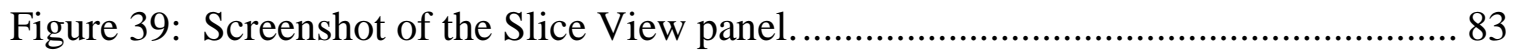

Figure 40: Adjusting Brightness and Contrast........................................................ 84

Figure 41: Pop-up menu to designate Calcification pattern.......................................... 85

Figure 42: Screenshot of a user annotating the Estimated Malignancy.......................... 86

Figure 43: Templates showing control points needed for AAM and ASM .................... 87

Figure 44: Modeling window that is triggered by clicking the Model button................ 88

Figure 45: Window used for obtaining manually annotated control-points. .................. 89

Figure 46: Viewing slice with previous tissue segmentation..................................... 91

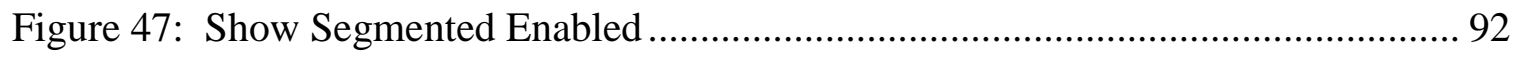

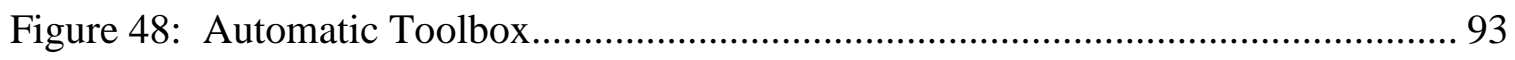

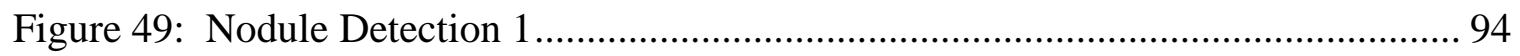

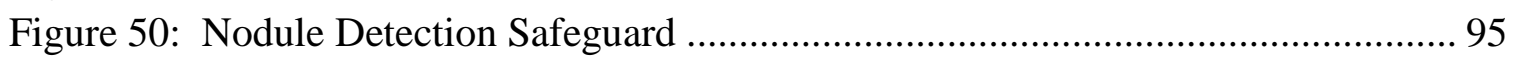

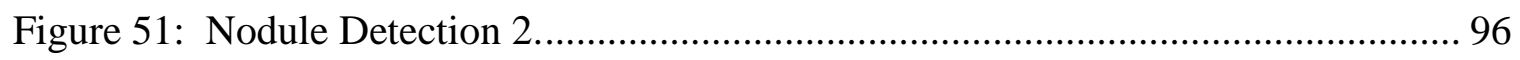

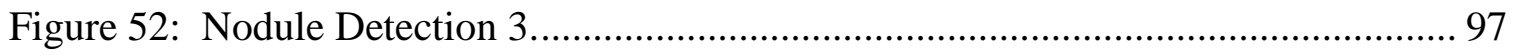

Figure 53: Classification, Segmentation and Modeling ........................................... 99 


\section{Introduction}

In 2010, the United States had an estimated 157,300 deaths due to lung cancer and a further estimated 222,520 newly diagnosed cases [7]. While these statistics present a high mortality rate, it should be further noted that the 5-year survival rate after resection of Stage 1A non-small cell lung cancer has been shown to be $62 \%-82 \%$ and in recent studies even $90 \%$ [2]. Stage 1A non-small cell lung cancer is defined as a malignant tumor, $3 \mathrm{~cm}$ or less in diameter, which has not yet spread to lymph nodes or surrounding tissues, [3]. By definition, this is categorized as a lung nodule; a mass of tissue in the lung with a diameter of $3 \mathrm{~cm}$ or less. The detection and treatment of Stage $1 \mathrm{~A}$ nonsmall cell lung cancer is highly correlated with patient survival and further, as a cancer advances to Stages 1B (tumors $>3 \mathrm{~cm}$ ) and beyond (spreading to surrounding anatomies), patient survival rates dramatically drop. Resection in these stages may become less effective and the cancer may become less responsive to alternative treatment techniques such chemotherapy or radiation therapy. While high survival rates for early lung cancer detection appear encouraging, approximately $0.2 \%$ of Computed Tomography (CT) scans (1 in 500) conducted in the U.S. reveal tumors at or less than 3 $\mathrm{cm}$ in diameter [2] and often, such tumors are found accidentally during screening for other abnormalities. This statistic is strikingly low and presents a paradoxical situation for approaching the problem of lung cancer detection. Do we introduce patients with a history of behaviors positively correlated with lung cancer, such as smoking, to more frequent CT scanning in a preventative fashion? This practice would, in turn, more frequently expose the patient to radiation used in the scanning process (which has been 
shown to increase the risk of cancer) with the hope of detecting possible nodules that are even difficult for a trained eye to identify. If a lung nodule is detected with a diameter between 5-10 mm, the recommended follow-up is 3, 6, 12, and 24-month serial CT scanning. This process becomes invasive for the patient, especially when the likelihood of malignancy is still highly uncertain.

On the clinical side, while many such nodules are benign, some may indicate a metastasizing cancer and thus, the timely identification and classification of all nodules within the lung tissue is essential to the survival of the patient. A principal limitation to identifying nodules is their limited spatial support—particularly when the motivation is early detection and the nodule may be closer to $1 \mathrm{~cm}$ in diameter. From an engineering standpoint, the introduction of a Computer Aided-Diagnostic (CAD) framework for addressing the detection and classification of lung nodules could be greatly beneficial to assisting radiologists for this problem. Harnessing the state-of-the-art, computational power of current Pattern Recognition, Machine Learning and Image Processing techniques could provide clinicians with an automatic, second-opinion detection and diagnosis schema. An immense disadvantage of employing such a CAD system is clinical validation, however the ability to computationally search CT scans for lung nodules using a process that is both extremely thorough and immune to human error is too valuable to not be exhaustively pursued. However, the CAD system proposed should be viewed as a diagnostic tool, not as a replacement for a trained radiologist.

A data-driven framework [8] has been proposed for the autonomous detection and classification of lung nodules from low-dose $\mathrm{CT}$ scans. This approach has been successful in categorizing lung nodules into four primary geometries (shapes), defined by 
[4], using Shape and Appearance Modeling approaches with high accuracy: WellCircumscribed, Juxta-Pleural, Pleural-Tail, and Vascularized. This detection and categorization has been achieved via the prior training of four mean nodule shapes from a selection of diverse, clinically extracted nodules [8]. From a global perspective, the proposed system can be illustrated as a simple pipeline and that pipeline has four main stages: Tissue Segmentation, Nodule Detection, Nodule Segmentation and Nodule Classification (as shown in Figure 1).

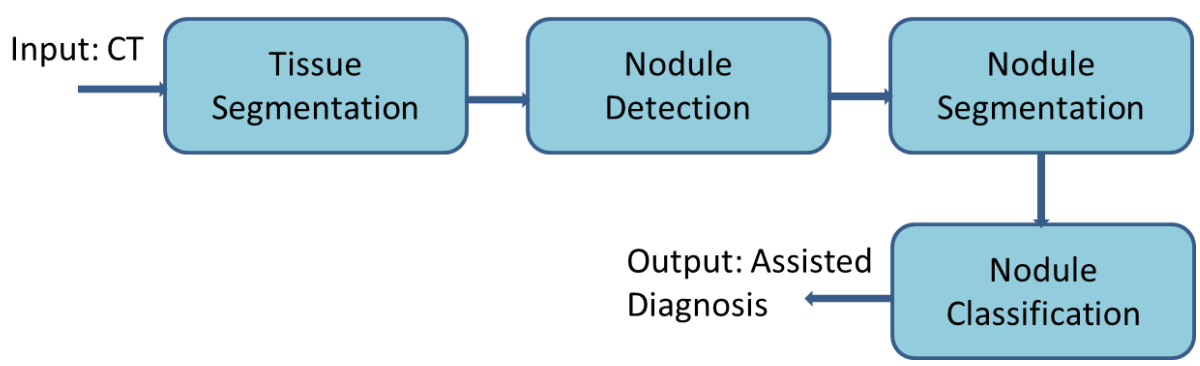

Figure 1: General CAD System pipeline shown to outline the overall process.

Starting from an input CT scan, the system first isolates the lung tissue from the extraneous $\mathrm{CT}$ information through the segmentation process. This is carried out to reduce the computational complexity of detecting the nodule in the second step by narrowing the region of interest to only the lung cavities. In the detection phase, a rasterstyle search is conducted to detect nodules using a template matching approach. The nodule models used in the template matching stage are generated offline using appearance and shape models that are constructed with a database of previously detected and annotated lung nodules. The crux of this process is the use of real lung nodule data to generate the models used in template matching. This data-driven approach is non- 
parametric and has been shown to outmatch parametric approaches for the application of lung nodule detection [5]. In order to accurately detect nodules, a sound mathematical definition must be formulated for both nodule shape and appearance. The non-parametric approach aims at extracting shape and appearance information from a real dataset of nodules, hoping to capture real-world variations in shape and texture for the process of modeling a search template. Firstly, the proposed framework follows the classification scheme of Kostis et al. [4], in which nodules are grouped into four main categories:

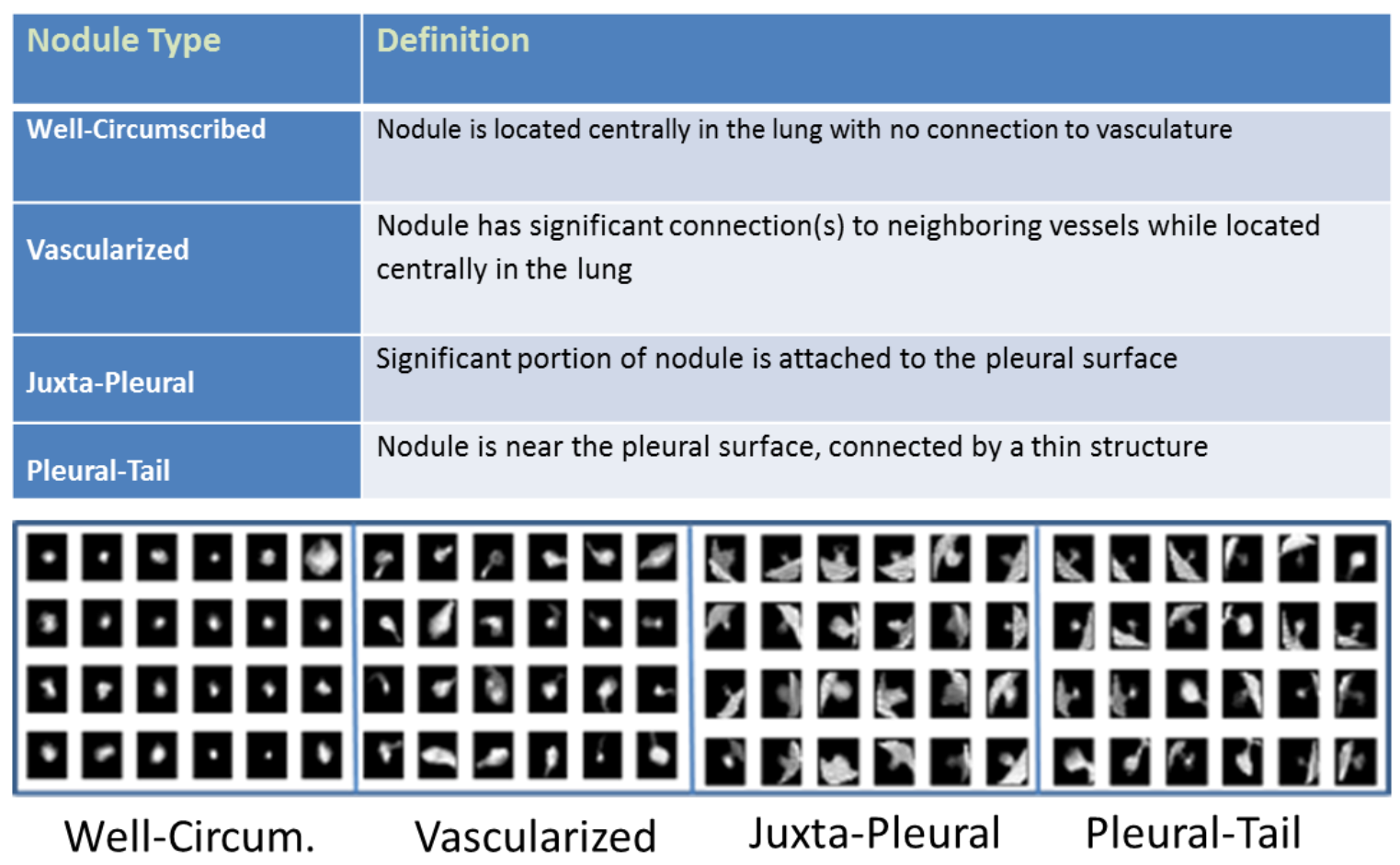

Figure 2: Four lung nodule types used in the proposed system. A visual sample of each type is provided below the definitions.

As shown above in Figure 2, this classification method is predominantly driven by shape. If one were to develop phantoms of each nodule type, distinguishing would be relatively 
straight-forward as shown in Figure 3. It should be noted that the nodule itself is distinct from surrounding anatomies such as vasculature or the pleural wall, however including extraneous anatomies in this classification scheme adds a significant amount of robustness to the template matching approach, as shown in later chapters. By inspection of the shape variations of each nodule type, one can see a distinct shape signature emerge for each nodule type.

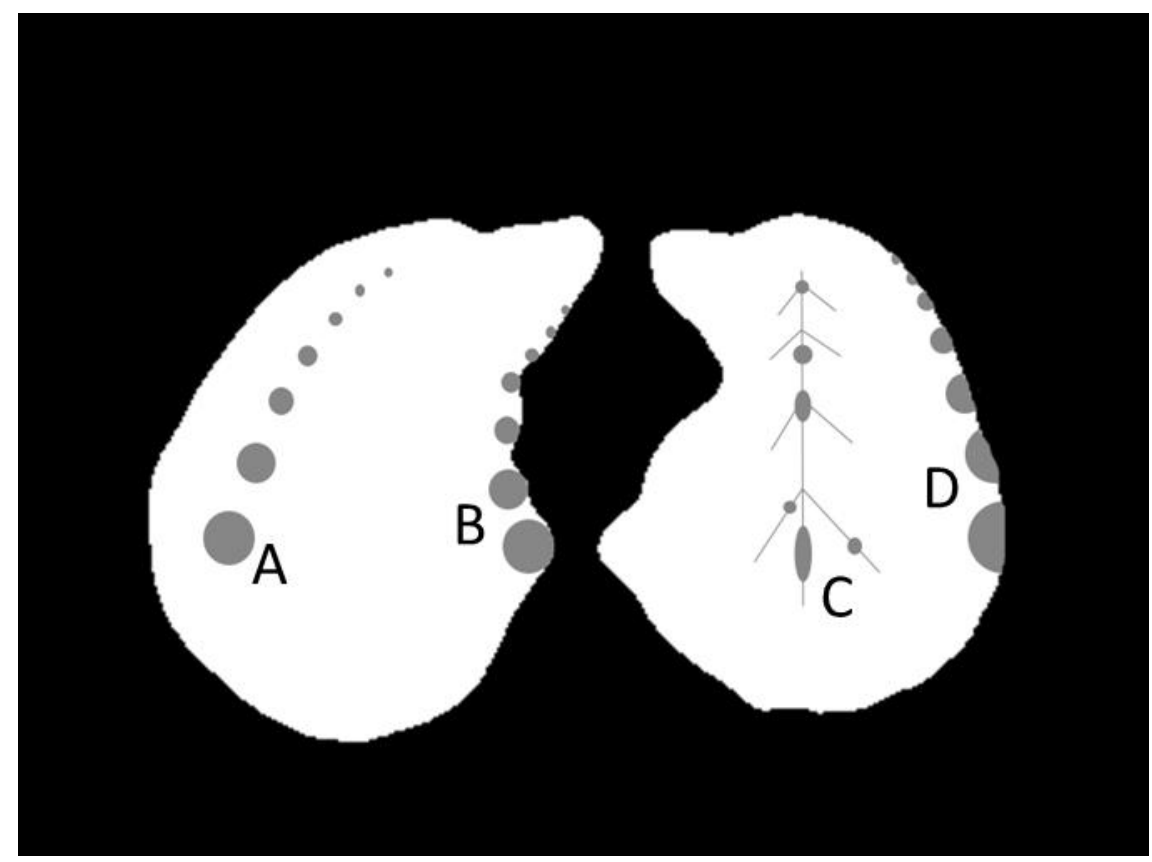

Figure 3: Phantom examples of the four nodule types according to the Kostis' classification framework. (A) Well-Circumscribed. (B) Pleural-Tail. (C) Vascularized. (D) Juxta-Pleural.

For completeness, Figure 4 illustrates examples of the four nodule types taken from two different datasets, the Early Lung Cancer Action Program (ELCAP) [6] and the Lung Image Database Consortium (LIDC) [10], thus showing a general uniformity of these nodule types across several sources. Understanding nodule shape and texture 
information via these four categories enhances the detection methodology by teaching the search algorithm locations in the lung cavity in which nodule likelihood is high and further, allows for a more exhaustive search for all possible nodule-like anatomies.

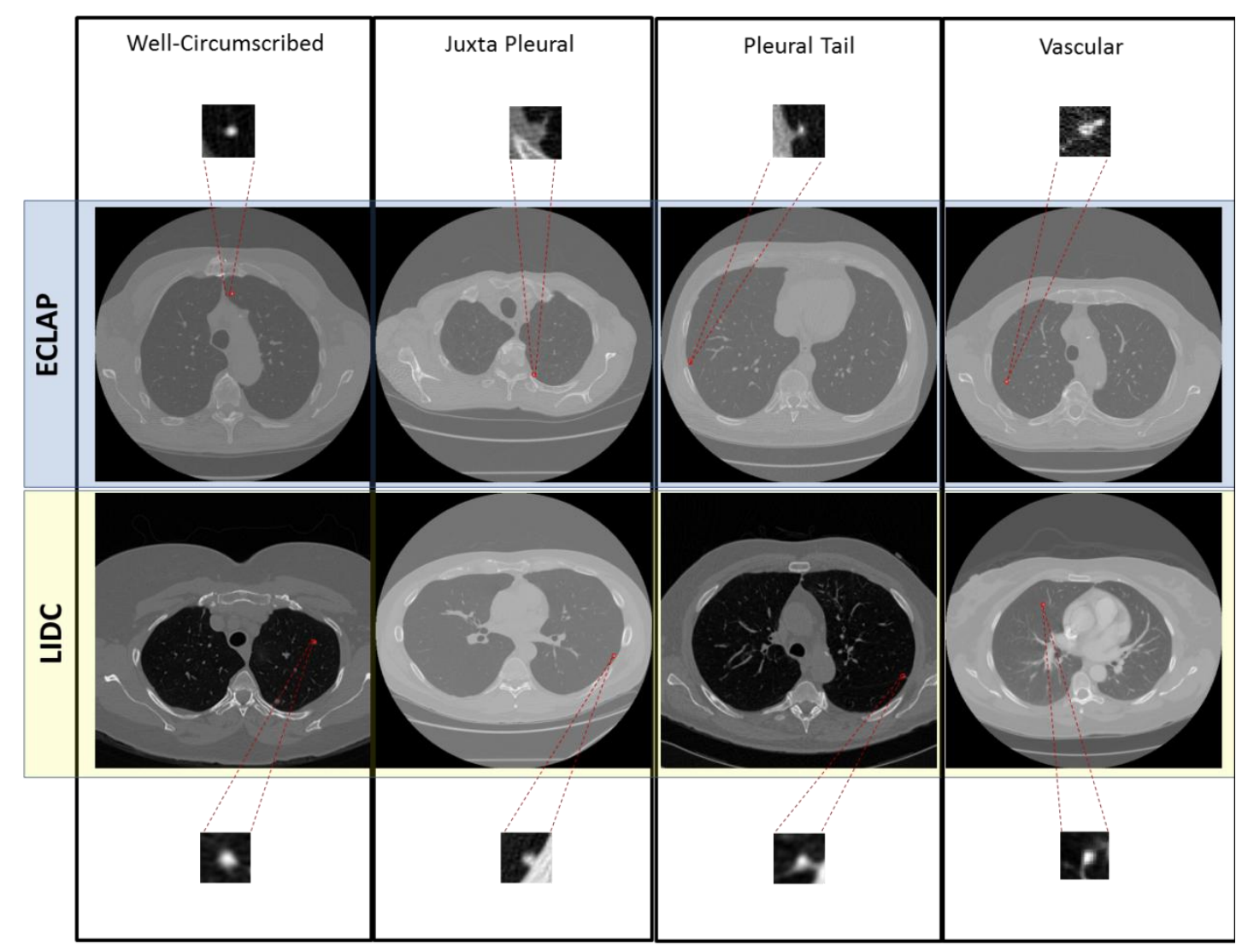

Figure 4: Further illustration of the Kostis' framework for lung nodule classification. Two examples of each type have been pulled from two independent datasets to emphasize how location and shape help define the four nodule categories.

The nodule detection phase passes potential nodule candidates to a second segmentation process in order to extract only those pixels belonging to the candidate nodule in question, removing all other anatomies surrounding (and possibly attached) to the candidate nodule. Obtaining a tight region that isolates the nodule in question provides 
input for the last stage of the pipeline: nodule classification. This stage attempts to classify the candidate in question into the categories of nodule or non-nodule.

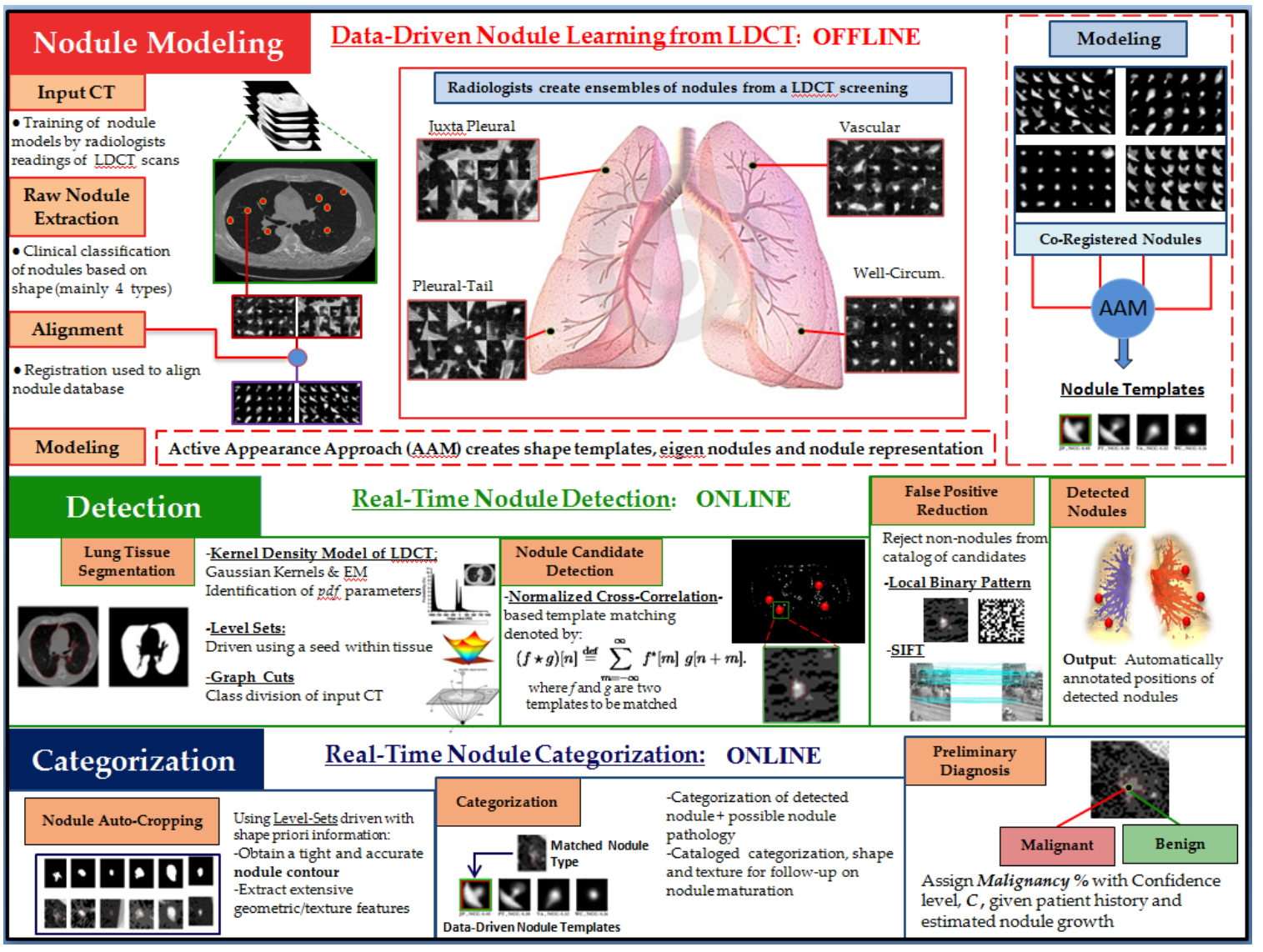

Figure 5: A more detailed outline of the CAD System. Tissue Segmentation is included in the Detection block of this diagram. Note that the modeling phase of this system is done offline and requires expert annotation of training nodules in order to drive the latter part of the system.

If the candidate is classified as nodule, further categorization is carried for the convenience of the user. The classification stage is multi-faceted in that a.) it acts as a false positive reducer, b.) it provides the user with a sample diagnosis based on previously classified lung nodules. 
The projected goal of the classification phase is to produce a malignancy metric with some confidence level given a detected nodule with the primary purpose of reducing the need for invasive biopsy/resection. A more detailed pipeline is shown in Figure 5. The system below has been designed to mimic the process of lung nodule detection as clinically performed by radiologists. Further, the pipeline is meant to enhance this process by introducing a fine, computational element — attempting to assist in cases where the trained, human-eye may question or even fail.

The specific goals of the CAD system are listed below:

1. Design a statistically significant database of nodules and a methodology to simulate possible uncertainties in lung nodules, in terms of size, location, shape and texture;

2. Design a feature detection approach, using topological object descriptors, in order to extract the features needed for categorization;

3. Implement a fast approach for matching using state-of-the-art machine learning algorithms, boosted by parallel implementation and a high-level language such as C\#

4. Lung nodule segmentation/cropping for enhanced shape modeling and growthrate measurement.

5. Validate the nodule categorization approach with respect to human experts.

The expected outcome is a computerized approach to detect and categorize nodules from CT scans (preferably low-dose) that will be beneficial for day-to-day readings of radiological scans, and for use in large scale studies aiming at early detection 
of lung cancer. The approach will also follow-up nodule growth and will assist in deciphering the tissue pathology, eventually reducing the need for biopsy. The CAD system will aim at overcoming several challenges, including:

- Resolution: Scale that corresponds pixel size w.r.t. to physical tissue dimension

- Contrast: Scale that measures the distinction between image components and appearance differences between classes of objects

- Sensitivity: Ability of the imaging protocol to enhance the contrast between anatomical features and non-anatomical features

- Specificity: Ability to distinguish pathologies from image information

- Noise: Corruption in image acquisition process realized as random fluctuations in image intensity

- Artifacts: Inaccuracies in visualization due to digitization/compression or acquisition process

- Occlusion/Distortion: Changes in shape, size, position, and other geometric characteristics (Nodules and anatomical structures are highly intermixed, thus false positives are inevitable)

- Spatial Support: Nodules are extremely subtle in some cases, providing little image information 


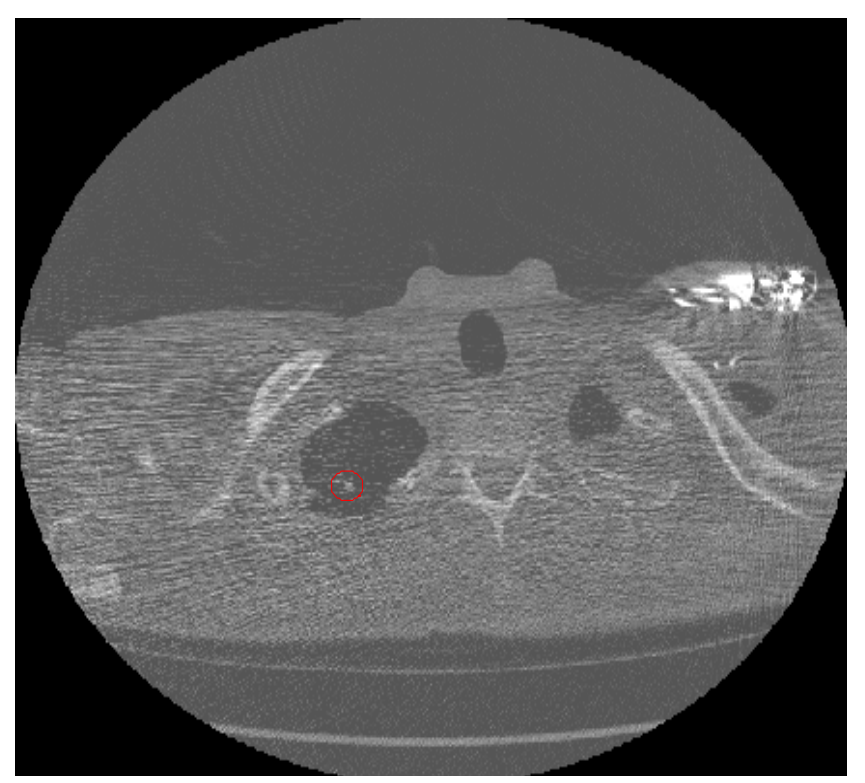

Figure 6: Example of a single slice taken from a noisy CT scan. The above slice illustrates problems with noise, artifacts, spatial support and resolution.

The objective of this thesis is to consolidate and enhance the existing tools relevant to the system pipeline above and answer the simple question: Can lung nodule detection truly be aided by an automated CAD system in real-world practice? While, for this thesis, the data-driven methodology and proposed system are aimed at lung nodules, this process may also be applied for any anatomy or abnormality studied in medical imaging and may prove to be an invaluable medical tool if engineered correctly. 


\section{Chapter I: Data Acquisition}

\subsection{Currently Available Lung Nodule Data}

As to be expected, a data-driven approach to modeling is entirely dependent on data acquisition. In order to generate robust and accurate nodule models with this approach, the core philosophy is simply "the more data, the more robust the model". The modeling phase in the proposed system needs to draw from a large population of nodules that effectively spans as much variation in nodule shape and texture (as has been clinically identified), thus enhancing the overall robustness of the models that follow. The two modeling approaches addressed in this project can be generalized as parametric and non-parametric. The parametric approach aims at synthesizing object models based on simple, known shapes and textures whereas the non-parametric approach is datadriven and generates object models using a collection of prior object realizations (realworld samples). The primary fallacy of the parametric modeling approach has been previously shown to be the incomplete definition of a lung nodule [20]. Simplifying computational nodule models to basic shapes and synthetic textures significantly degrades the overall detection and classification outcomes in subsequent steps of the proposed system. Therefore, if the goal of developing this system is enhancing the clinical reach of LDCT and providing an assisted diagnosis, the non-parametric approach should be adopted. Computationally learning nodule shape and texture via monitoring and mimicking expert clinicians is the most effective route for achieving this goal. 
Previous work in this area has been dependent on publicly available lung nodule databases. The most notable datasets that have been employed by the CVIP Lab are the ELCAP and the LIDC. Further, the CVIP Lab has begun collecting data from collaborating physicians in both Louisville, Kentucky (Jewish) and Mansoura, Egypt (اللمد ذصورة). A brief overview of these datasets is provided in Table 1.

\begin{tabular}{|c|c|c|c|c|}
\hline Database & ELCAP & $\begin{array}{l}\text { Jewish } \\
\text { Hospital } \\
\end{array}$ & الـ ذـ صورة & LIDC \\
\hline \# Nodules & 397 & 112 & 50 & 2669 \\
\hline \# Patients & 50 & 10 & 6 & 1010 \\
\hline $\begin{array}{l}\text { Size Range } \\
(\mathbf{m m})\end{array}$ & 3 to 5 & 3 to 21 & 3 to $>40$ & 3 to 27 \\
\hline $\begin{array}{l}\text { Expert Nodule } \\
\text { Segmentation }\end{array}$ & No & No & No & Yes \\
\hline \# Clinicians & N/A & 1 & 1 & 12 \\
\hline $\begin{array}{l}\text { Multiple Slices } \\
\text { Per Nodule }\end{array}$ & No & No & No & Yes \\
\hline $\begin{array}{l}\text { Classification } \\
\text { Offered }\end{array}$ & No & Yes & Yes & Yes \\
\hline $\begin{array}{l}\text { Retrospective } \\
\text { Study }\end{array}$ & No & No & No & $\begin{array}{r}\text { Accidentally } \\
\text { (No Biopsy) }\end{array}$ \\
\hline $\begin{array}{l}\text { Multiple Scans } \\
\text { Per Patient }\end{array}$ & No & Yes & No & No \\
\hline $\begin{array}{l}\text { Scans With } \\
\text { Radiocontrast } \\
\text { Agent }\end{array}$ & Yes & Yes & Yes & Yes \\
\hline $\begin{array}{l}\text { Slice Thickness } \\
(\mathrm{mm})\end{array}$ & 1.25 & 1.5 to 2.5 & 1.5 to 2.5 & 0.6 to 5.0 \\
\hline
\end{tabular}

Table 1: Overview of the lung nodule databases used in previous work leading up to the design of the proposed system. Both ELCAP and LIDC are publicly available, while the other two have been started as part of an effort by the CVIP Lab to build a larger, more dynamic public dataset of lung nodules. 
Table 1 outlines some features of the nodule datasets currently used in the data-driven modeling process. There are roughly 3200 nodules in total in combining the datasets. As shown, the publication of the LIDC dataset has provided a significant portion of the currently used working nodule population for this system. Prior to the LIDC, the modeling phase of the proposed system was dependent on a population of well under 1000 nodules. From a machine-learning standpoint, this is highly inadequate for the purposes of capturing accurate, real-world, lung nodule statistics. The LIDC is also the only dataset to provide ground truth, expert outlines of enrolled nodules. Further, each enrolled nodule is annotated and outlined in multiple slices allowing for the extension into 3-D. The LIDC also trumps the other datasets in an increased number of nodules, from an increased number of patients with up to 4 expert opinions per nodule. In pursuit of accurate nodule shape, expert outlines are priceless for the purposes of modeling as well as validation of automatic nodule segmentation techniques. Acquiring such an extensive dataset involves overcoming several hurdles in the temporal, financial, and scientific domains. The primary constraint in the process of building a lung nodule database is attaining a clinical consensus on those nodules that are enrolled. Figure 7 shows the discrepancy in expert outlines provided by the LIDC for each of the four nodule types. The outlines provided are all for nodules that are $>10 \mathrm{~mm}$ in diameter and they illustrate a rough consensus on nodule boundary. However, from a machinelearning point of view, these contours vary significantly and for smaller nodules in the LIDC, the variability in nodule contour greatly increases.

While the LIDC may be labeled as the current gold standard in lung nodule databases it has many short-comings. The system designed in this thesis aims allowing 
collaborating radiologists to continuously add to a dynamic nodule database, which can be used for both the construction of a larger, more robust publicly available database as well as personal use by radiologists to assist their diagnosis.
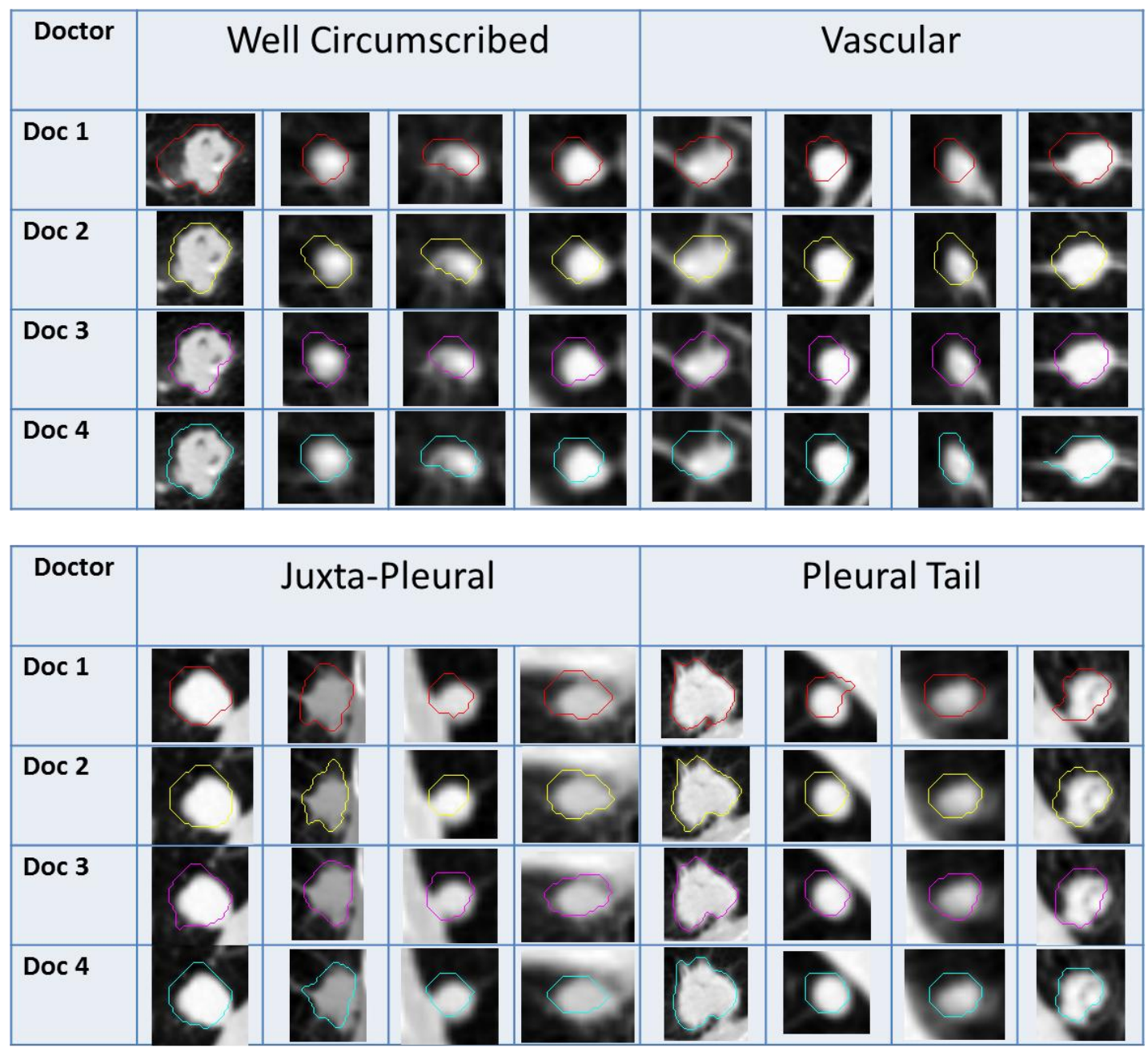

Figure 7: Sample of expert outlines provided by 4 radiologists to illustrate discrepancy in nodule contour for each of the four nodule types. While the contours appear very similar, from a machinelearning standpoint they greatly vary given the low spatial support of lung nodules. Each expert has outlined the same nodules.

One goal of the CAD system is to generate dynamic, data-driven models via radiologist(s) annotation for the successive detection and classification of future lung 
nodules, i.e. personalized machine learning of a radiologist's nodule detection technique. Further, obtaining a sub-population of nodules in a retrospective fashion will allow for the extension of the data-driven approach into modeling nodule malignancy. It should be noted that none of the datasets provide biopsy information for the purposes of tracking malignant specimens. In tracking nodule history in revisiting patients, the modeling schema can be extended to model both malignancy and benignity for the future classification of nodules with an uncertain level of malignancy.

\subsection{Data Acquisition in CAD System}

The CAD system designed in this thesis aims at providing a functional and efficient data acquisition scheme for operating physicians. To accomplish this, the following classification protocol has been established for enrolling newly annotated nodules into the working database:

\begin{tabular}{l|l|l}
\hline Feature & Description & Options \\
\cline { 3 - 3 } Location & $\begin{array}{l}\text { X, Y coordinates of } \\
\text { annotated nodule }\end{array}$ & $\begin{array}{l}\text { (Obtained from initial } \\
\text { annotation) }\end{array}$ \\
\hline Slice Number & $\begin{array}{l}\text { Slice number containing the } \\
\text { annotated nodule according } \\
\text { to the sorted scan }\end{array}$ & $\begin{array}{l}\text { (Obtained from initial } \\
\text { annotation) }\end{array}$ \\
\hline $\begin{array}{l}\text { Anatomical } \\
\text { Location }\end{array}$ & $\begin{array}{l}\text { Anatomical region of the } \\
\text { lung in which the annotated } \\
\text { nodule was detected }\end{array}$ & $\begin{array}{l}\text { 1. Central } \\
\text { 2. Peripheral }\end{array}$ \\
\hline
\end{tabular}




\begin{tabular}{|c|c|c|}
\hline Morphology & $\begin{array}{l}\text { Information regarding the } \\
\text { contour of the annotated } \\
\text { nodule }\end{array}$ & $\begin{array}{ll}\text { 1. } & \text { Smooth/Round } \\
\text { 2. } & \text { Lobulated } \\
\text { 3. } & \text { Spiculated } \\
\text { 4. } & \text { Aytpical }\end{array}$ \\
\hline Subcategory & $\begin{array}{l}\text { Category in which the } \\
\text { annotated nodule belongs } \\
\text { according to the Kostis' } \\
\text { classification standard }\end{array}$ & $\begin{array}{l}\text { 1. Well-Circumscribed } \\
\text { 2. Juxta-Pleural } \\
\text { 3. Pleural-Tail } \\
\text { 4. Vascularized } \\
\text { 5. None }\end{array}$ \\
\hline Calcification & $\begin{array}{l}\text { Pattern in which calcium } \\
\text { deposits appear within the } \\
\text { annotated nodule }\end{array}$ & $\begin{array}{l}\text { 1. Popcorn } \\
\text { 2. Laminated (Concentric) } \\
\text { 3. Diffuse } \\
\text { 4. Central } \\
\text { 5. Completely Calcified } \\
\text { 6. Partially Calcified } \\
\text { 7. Non-Calcified } \\
\text { 8. Fat } \\
\text { 9. Other }\end{array}$ \\
\hline Attenuation & $\begin{array}{l}\text { Pattern in which radiation is } \\
\text { absorbed by annotated } \\
\text { nodule }\end{array}$ & $\begin{array}{ll}\text { 1. } & \text { Solid } \\
\text { 2. } & \text { Mixed-Solid } \\
\text { 3. } & \text { Ground Glass } \\
\text { 4. } & \text { Other }\end{array}$ \\
\hline $\begin{array}{l}\text { Estimated } \\
\text { Malignancy }\end{array}$ & $\begin{array}{l}\text { Estimation from user } \\
\text { regarding the possibility of } \\
\text { malignancy in the annotated } \\
\text { nodule }\end{array}$ & $\begin{array}{l}\text { Percentage ranging from } 0 \text { to } \\
100 \% \text { with incremental steps of } \\
1 \%\end{array}$ \\
\hline
\end{tabular}

Table 2: Data acquisition scheme for enrolling newly annotated nodules into the working database. Each option presented is explicit, meaning only one may be chosen by the user.

These acquisition criteria have been selected based on CVIP collaboration with expert physicians/radiologists and outline a robust signature for each newly enrolled nodule that has been manually annotated by a physician using the CAD system. The first two features, Location and Slice Number are obtained from an initial point and click by the reading physician where the nodule has been found and are stored for later referencing. Anatomical Location describes the region of lung in which the annotated 
nodule was found. Centrally located nodules exist within the lung parenchyma (bulk of the organ), peripheral nodules exist on the edge of the parenchyma and subpleural nodules exist between the pleura and parenchyma. Morphology addresses the geometric features of the annotated nodule's outermost contour. The options available for morphologic classification are listed in ascending order of suspicion. Nodules contours that exhibit low change in curvature and appear smooth have a lower likelihood of malignancy. Likewise, nodules with contours that have corona radiata appearance or appear as a sunburst pattern (high changes in curvature) are generally more likely to be malignant. The medical definition of corona radiata is an encircling structure that resembles a crown. See Figure 8 below:
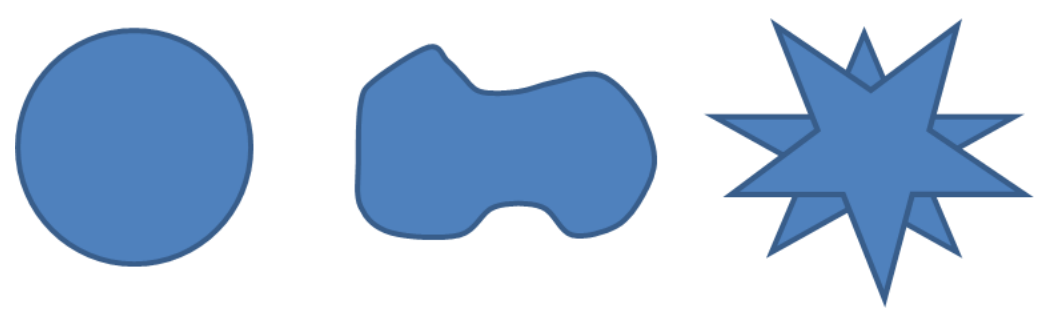

Figure 8: Synthetic examples of contours corresponding to nodule morphology. (Left) Smooth/Round, (Middle) Lobulated, and (Right) Spiculated.

The Subcategory refers to the Kostis' classification scheme discussed above: Well, Juxta, Tail and Vascular. The user can opt to categorize the annotated nodule as Other for this feature if ambiguity or uncertainty exists. Calcification is generally a more reliable indicator of benignity [21]. Here, the user is polled to provide information regarding the pattern with which calcium deposits occur in the annotated nodule, which is determined by inspecting the spatial distribution of the Hounsfield Units (HU). Nodule 
calcification generally appears in distinct patterns, some of which are associated with benignity. See Figure 9 below. Popcorn calcification is usually an indication of hamartomas, which are benign and grow at the same rate as the lung tissue. Likewise, laminated, central and diffuse calcification patterns can be indications of granulomas, which are inflammations caused by the body's attempt to isolate a growth that is identified as foreign. Granulomas are also benign in nature. The remaining options for this feature allow the user to assign a calcification pattern to an annotated nodule that has a likelihood of malignancy, including Non-Calcified.
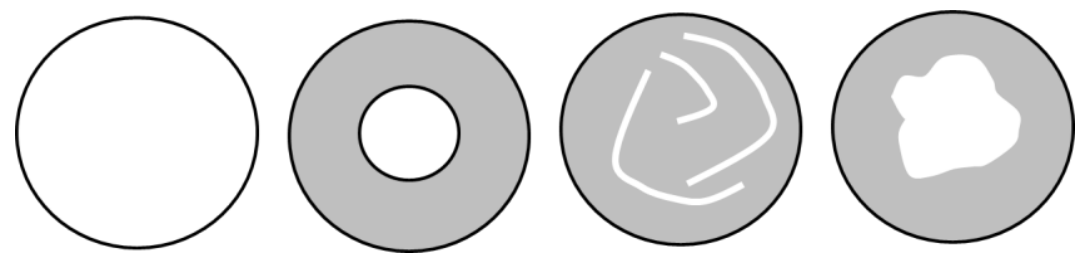

Figure 9: Synthetic examples of benign calcification patterns. (Left) Diffuse, (Second) Central, (Third) Laminated, and (Right) Popcorn.

Attenuation addresses the opacity of annotated nodule and is related to the density of the lesion, providing a metric of radiation absorption. In terms of imaging, this is visually realized as the uniformity and opaqueness of a nodule's intensity. Solid attenuation appears as more uniform, opaque intensity whereas Ground Glass refers to a more inhomogeneous and translucent intensity. Ground Glass nodules are generally higher indicators of malignancy. The final feature assessed by the user is Estimated Malignancy. This allows the physician to estimate malignancy percentage on a scale of $0-100 \%$ in discrete, $1 \%$ increments. This value is strictly an estimate unless the nodule 
has a history of biopsy, but allows the system to analyze correlations between estimated malignancy and the features described above.

Chapter 6 outlines how the system has been designed in order to obtain these annotated metrics from operating physicians, including real-time user interaction as well as the scheme for organizing and storing nodule data. 


\section{Chapter 2: Tissue Segmentation}

\subsection{Overview and Motivations}

Segmentation is a well-investigated problem in image processing and yet, given the number of expansive and diverse approaches available, it still remains unsolved. The ambiguity of segmentation lies in its definition, which for a large number of cases, is application-based. The literature on this issue is thorough and extensive, providing a wide variety of segmentation solutions for an even wider array of scenarios.

Segmentation is defined as the following:

Segmentation - The process of partitioning a signal into individual regionseach with varying levels of interest depending on the application.

For this component of the system, our region of interest is that bounded by the tissue contour.
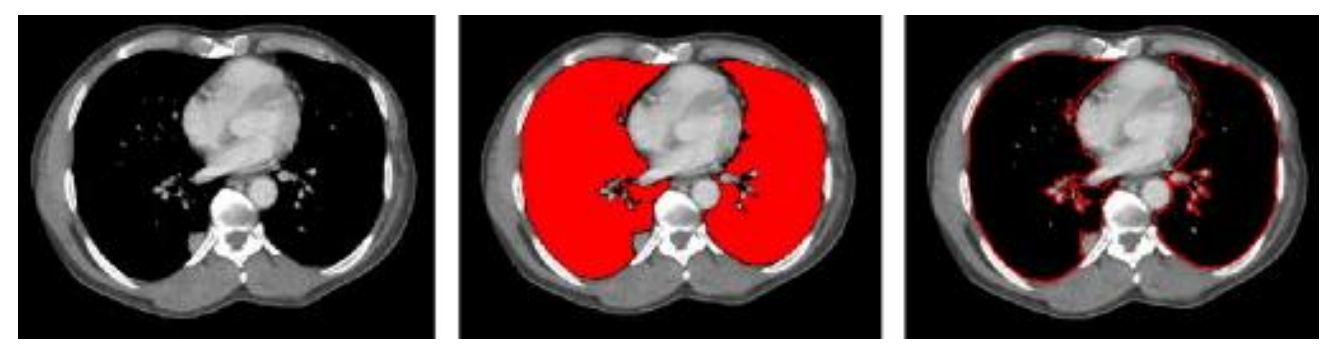

Figure 10: Visual illustration of the segmentation of lung tissue from raw CT. (Left) Raw Chest CT. (Middle) Red region highlights segmented lung tissue. (Right) Lung tissue boundary obtained from segmentation process outlined in red. Image taken from [9].

The goal of tissue segmentation is to reduce the search domain for detecting lung nodules. Removing various anatomies such as the heart, ribs, and fluids, reduces the 
search domain to only the lung tissue, known to contain lung nodules. This preprocessing procedure greatly aids the overall computational efficiency of the system and only needs to be conducted once per scan, provided the results are desirable to the user. Saving and retrieving segmentation results of a previous scan is simple task and a functional background component of the overall system.

Although there are countless available options for this task, we have employed a selection of simple methods with the motivation of keeping computation time to an absolute minimum. For the proposed CAD system, the tissue segmentation approaches have been empirically chosen to be the 3D Level Set method and the Expectation Maximization method—-both of which minimize the need for user interaction and yield results less sensitive to variations in CT scanning protocol.

\subsection{Expectation Maximization Algorithm}

The Expectation Maximization (EM) algorithm is an iterative procedure for finding maximum likelihood estimates of parameters of statistical processes in cases where the process depends on hidden, random variables. The EM algorithm iteratively alternates between an expectation step and a maximization step. The expectation step finds the expectation of the log-likelihood current parameter estimates while the maximization step maximizes the expected log-likelihood produced in the expectation step. This process leapfrogs back and forth until converging to stable parameter estimates, which describe the statistical process. 
Assume a set of observed random variables, $\mathbf{X}$, and a set of hidden random variables, $\mathbf{Z}$. These variables have a likelihood $p(\boldsymbol{X}, \boldsymbol{Z} / \boldsymbol{\theta})$, where $\boldsymbol{\theta}$ represents an unknown vector of parameters needed to describe $p$. The maximum likelihood estimate (MLE) of $\boldsymbol{\theta}$ is given by:

$$
p(\boldsymbol{X}, \boldsymbol{\theta})=\sum_{\boldsymbol{Z}} p(\boldsymbol{X}, \boldsymbol{Z} / \boldsymbol{\theta})
$$

The MLE can be numerically computed using the EM algorithm. The first step is the expectation step, which computes the expected value of the log likelihood function with respect to the current estimated parameter vector, $\boldsymbol{\theta}^{+}$.

$$
Y\left(\theta / \theta^{+}\right)=E_{\boldsymbol{Z} / \theta^{+}}[\log (p(\boldsymbol{X}, \boldsymbol{Z} / \boldsymbol{\theta}))]
$$

The maximization step of this algorithm, finds the parameter vector $\boldsymbol{\theta}^{\prime}$ such that $Y$ max:

$$
\boldsymbol{\theta}^{\prime}=\operatorname{argmax}_{\boldsymbol{\theta}}\left[Y\left(\theta / \theta^{+}\right)\right]
$$

If the form of $p$ is assumed to be a Gaussian Mixture Model (GMM) [ref GMM], $\boldsymbol{\theta}$ (mean and covariance) based on the empirical analysis of chest CT histograms, the EM algorithm can be adequately applied to isolating the intensity bandwidth that belongs to the lung tissue. For a more in depth derivation, see [42]. 


\subsection{Level Sets}

A level set function $\Phi: \Omega \subset R^{2} \rightarrow R$ can be cast as the minimum distance between any pixel $x \in \Omega$ and the boundary pixels of a given object. This information can be captured with a Signed-Distance Map (SDM), shown in Figure 11. Using image intensity, an initial contour evolves in an attempt to fit to the true object contour.

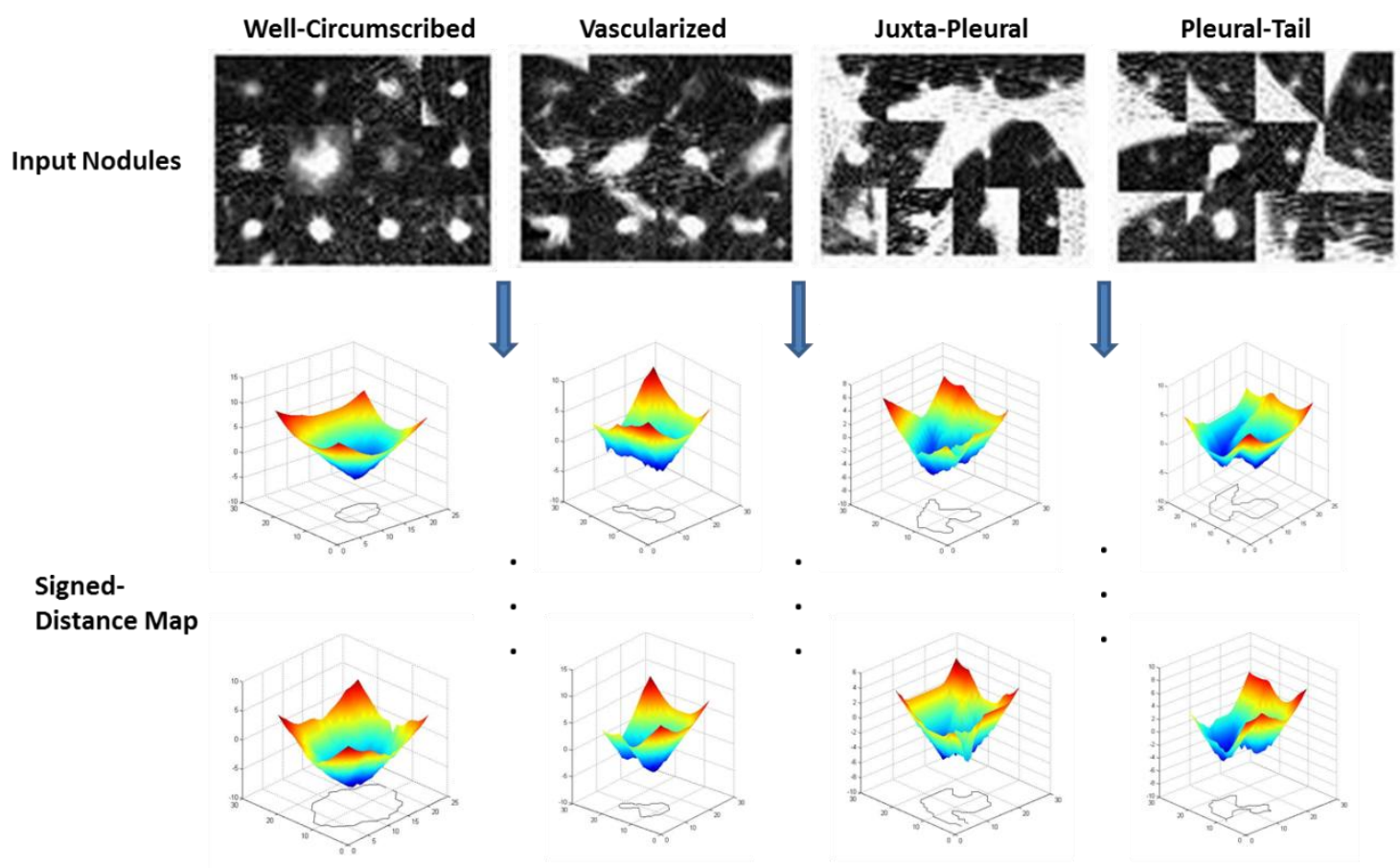

Figure 11: Sample signed-distance maps of the four nodule categories. These maps are used to build the prior shape model and during the process of nodule segmentation to drive the propagating front. This figure pertains to nodule segmentation, however it illustrates the meaning of the Signed-Distance Map.

Given an image $I: \Omega \subset R^{2} \rightarrow R$, the tissue segmentation process aims to partition the given CT slice into two regions: tissue, (denoted as $t$ ) and background (denoted as $b$ ). A contour will be obtained, which bounds all pixels $\epsilon t$. An error metric is employed to 
drive the process, which counts the number of correctly classified pixels as compared to the total number of pixels in the image.

This error metric is given by:

$$
e=1-\pi_{t} \int_{\Omega_{t}} p_{t}(I(\boldsymbol{X})) d \Omega-\pi_{b} \int_{\Omega_{b}} p_{b}(I(\boldsymbol{X})) d \Omega
$$

$p_{t}$ and $p_{b}$ represent the probability of intensity for both tissue and background, respectively. $\pi_{t}$ and $\pi_{b}$ represent the prior probabilities of tissue and background, respectively. It should be noted that the intensity distributions of both tissue and background are assumed to be Gaussian based on the evident histogram modes present in Chest CT. Contour evolution is based on minimizing the following energy functional:

$$
E(\phi)=-\pi_{t} \int_{\Omega_{t}} p_{t} H_{\epsilon}(\phi) d \Omega-\pi_{b} \int_{\Omega_{b}} p_{b} H_{\epsilon}(-\phi) d \Omega
$$

where $H$ is the Heaviside step function and $\epsilon \in R^{+}$represents the narrow band region around the current zero-level, $\phi$. For the purposes of smooth evolution, the contour arclength, $L$ is also considered for minimization:

$$
E(\phi)=-\pi_{t} \int_{\Omega_{t}} p_{t} H_{\epsilon}(\phi) d \Omega-\pi_{b} \int_{\Omega_{b}} p_{b} H_{\epsilon}(-\phi) d \Omega+\lambda L
$$

where $\lambda$ represents a smoothing factor. Minimization of this term is carried out using the Euler-Lagrange formulation with the gradient descent optimization:

$$
\frac{\partial \phi}{\partial t}=\delta_{\epsilon}(\phi)\left(\pi_{t} p_{t}-\pi_{b} p_{b}\right)+\lambda \kappa
$$

where $\delta$ is the derivative of the Heaviside function and $\kappa$ is the curvature. Iteratively solving $\phi(X, t)=0$, obtains the current evolving contour. 
For the application of lung tissue segmentation, the level-set method above is computationally expensive. The recursive computation of the signed-distance function is a computationally strenuous process — especially when the object of interest accounts for a large part of the image domain, such as with the lung tissue. Due to the appearance of lung tissue in $\mathrm{CT}$, this process can be abridged to the following algorithm:

1.) Initialize the object of interest: $\Phi_{\text {Init }}$ with known seed points:

a.) Generate an inital object mask such that: Pixels $\in$ Initial Object $=1$ (pixels bounded by $\Phi_{\text {Init }}$ ), and Pixels $\notin$ Initial Object $=0$ (pixels outside $\Phi_{\text {Init }}$ )

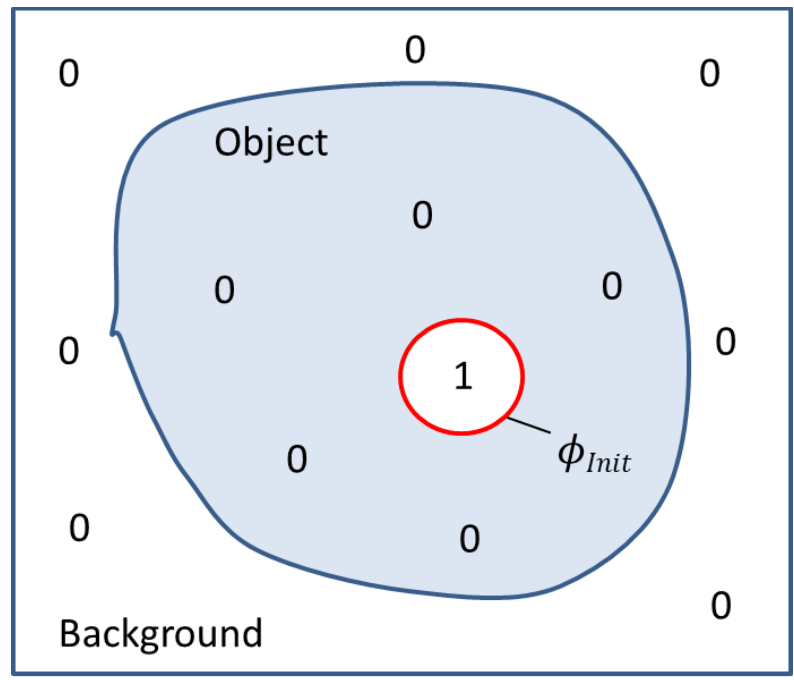

Figure 12: Initial object contour obtained from known seed points within the region of interest. These seed points come from contextual cues, pre-processing or manual interaction. Illustrated in 2D but may easily be extended to 3-D.

2.) Let Object $=$ Initial Object , and $\Phi=\Phi_{\text {Init }}$. Iterate:

3.) Obtain the 3-D edge map of $\Phi$ : 


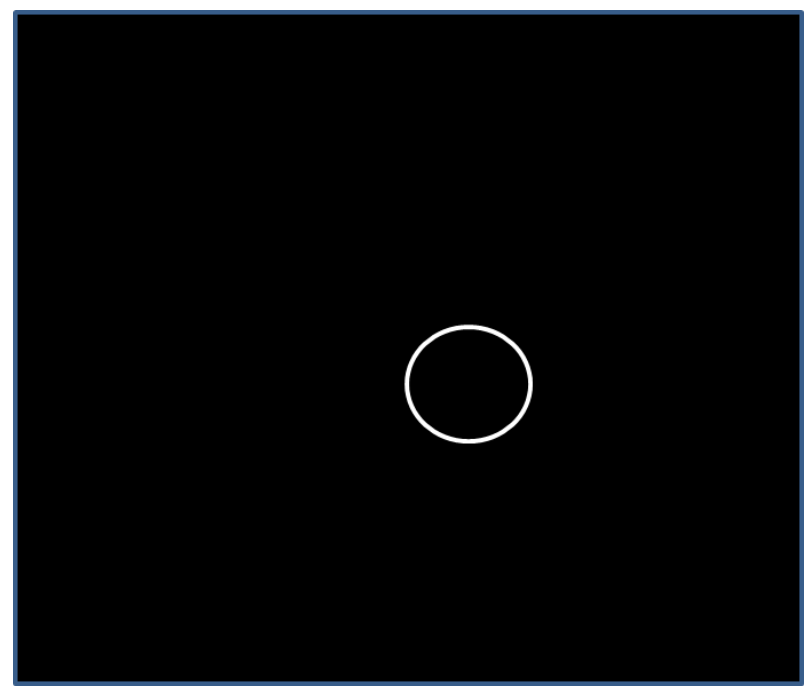

Figure 13: Binary edge map of the initial object region. Pixels on the contour have a grayscale intensity of $\mathbf{2 5 5}$ and all remaining pixels have a grayscale intensity of 0.

4.) Narrow Band the 3-D edge map:

a.) Grow the edge map a distance of 1 pixel in all directions and store as Narrow Band

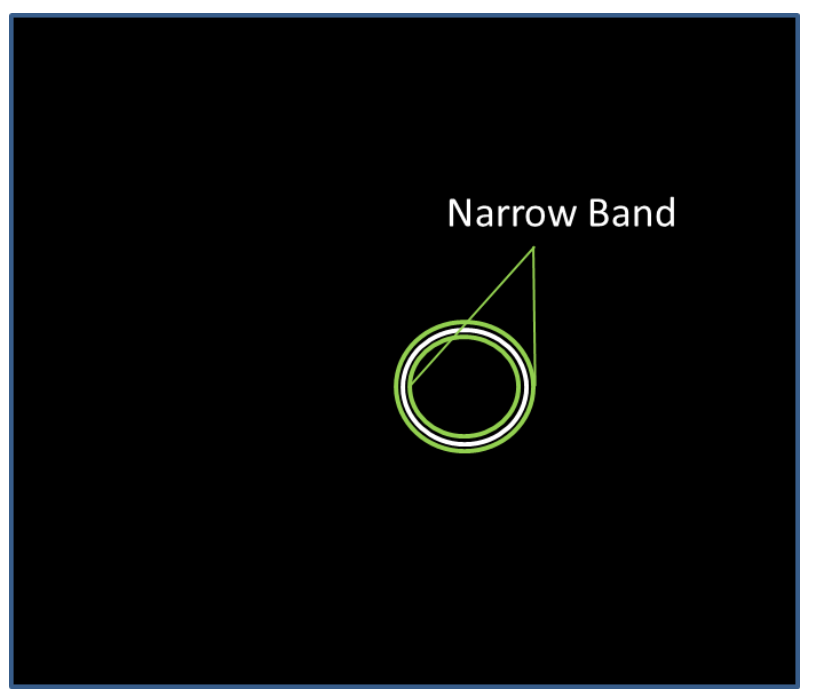

Figure 14: The narrow band consists of all pixels neighboring the contour in the edge map. The narrow band region houses all candidate pixels which may be accepted as belonging to the object of interest or rejected and classified as background pixels. 
5.) Update $\Phi \rightarrow \Phi_{+}$:

a.) Calculate the mean intensity of pixels belonging to $\Phi$ and the mean intensity of the remaining background pixels, $\mu_{\text {Object }}$ and $\mu_{\text {Background }}$

b.) For each pixel $\boldsymbol{x} \in$ Narrow Band with intensity $I(\boldsymbol{x})$, Calculate: $\xi_{0}=$ $\left|I(\boldsymbol{x})-\mu_{\text {Object }}\right|$ and $\xi_{B}=\left|I(\boldsymbol{x})-\mu_{\text {Background }}\right|$.

If $\xi_{O} \leq \xi_{B}: \Phi(\boldsymbol{x})=1$, else: $\Phi(\boldsymbol{x})=0$

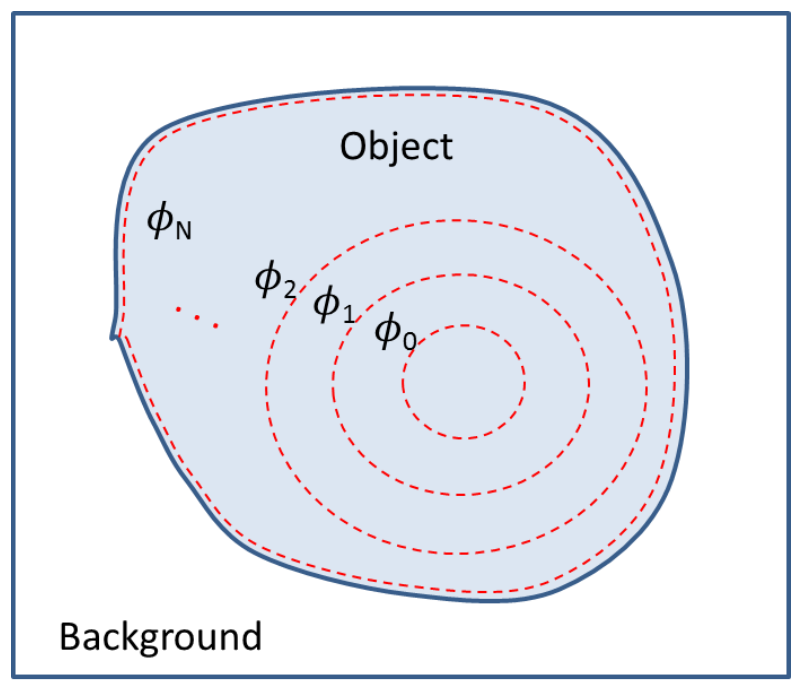

Figure 15: Illustration of $\Phi$ converging to the true object contour. Evolution is terminated by pre-determining the number of iterations $\Phi$ may undergo or by monitoring a lack of significant change between consecutive iterations.

6.) (Optional) Smooth $\Phi$ using 3-D median filter

7.) If Iteration \# > Termination \#, return $\Phi$

Else Jump to 3.) 
Applying the above algorithm to an input image will output a final object mask, in which pixels belonging to the object of interest are labeled as 1 (White) and pixels belonging to the background are labeled as 0 (Black).

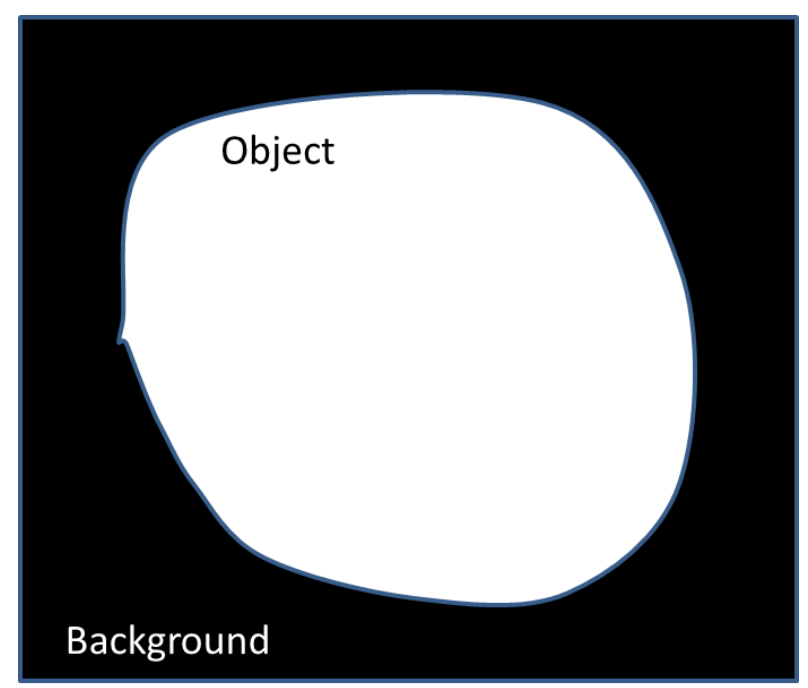

Figure 16: Output of statistical level sets algorithm. The resulting binary mask consists of two classes, Object and Background. This mask is used to isolate true object pixels in the original image.

Some sample results of the algorithm applied on Chest CT images are shown below in Figure 17. The abridged algorithm is sufficient for segmenting the tissue from the Chest CT - primarily due to strong, well-defined edges along the pleural wall and the distinct modes apparent in the histogram of a Chest CT slice. The lung tissue is statistically separable from other anatomies in the image and thus, the algorithm performs well. However, there is one major downfall to this approach: initialization. The level-set segmentation process is extremely dependent on initialization. For implementing this approach in the overall system, this leaves two options: 1.) rely on manual seeding of the lung tissue by the user or 2.) use an automatic approach to obtain seed pixels which are 
known to belong to the lung tissue. Both options are made available to the user in the proposed system. The EM algorithm, as described above, is used as the automatic seeding solution. The EM segmentation process is applied to the median slice within a scan, providing an initial 2-D mask. Using the same labeling scheme as described above, the centroids of both the left and right lung can be estimated. The two centroids and a narrow-band region around each are treated as seed points within the tissue in order to generate $\Phi_{\text {Init }}$
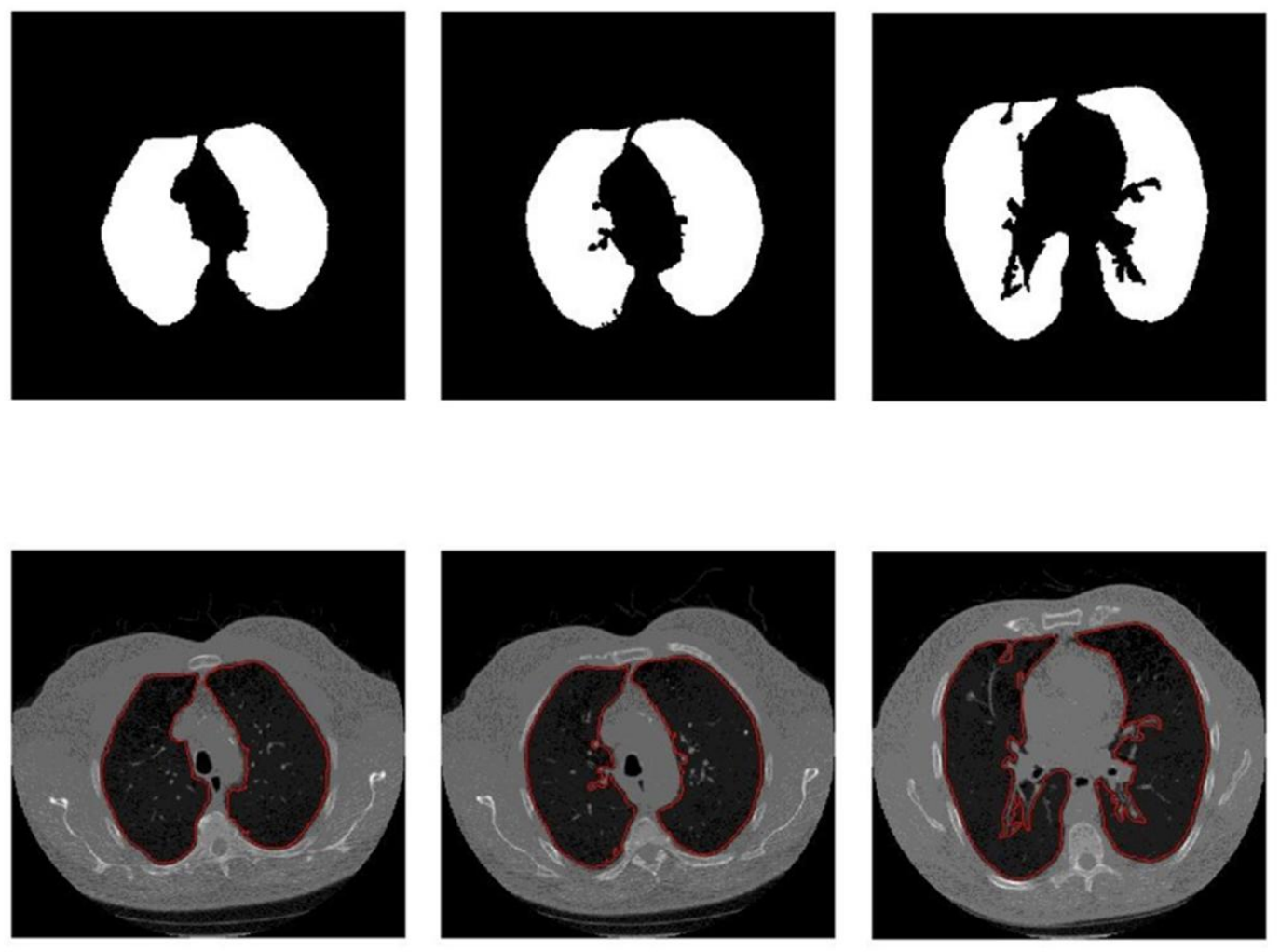

Figure 17: Sample, 2-D projections obtained from 3-D Statistical Level-Sets algorithm. (Top row) Binary object masks isolating lung tissue. (Bottom Row) Original CT images with final contour shown in red. 


\section{Chapter 3: Nodule Detection}

\subsection{Overview, Motivations and Challenges}

The goal of CAD system development for lung nodules is assisted diagnosisnamely, early detection. There are several challenges to overcome when addressing early nodule detection. As previously discussed, nodule spatial support is a significant limitation when applying pattern recognition methodologies. Noise, high slice thickness, low image resolution, small nodule diameter, high variation in nodule shape and appearance, high variation in nodule intensity distribution and occlusion all greatly affect the process of accurately modeling and automatically detecting lung nodules.

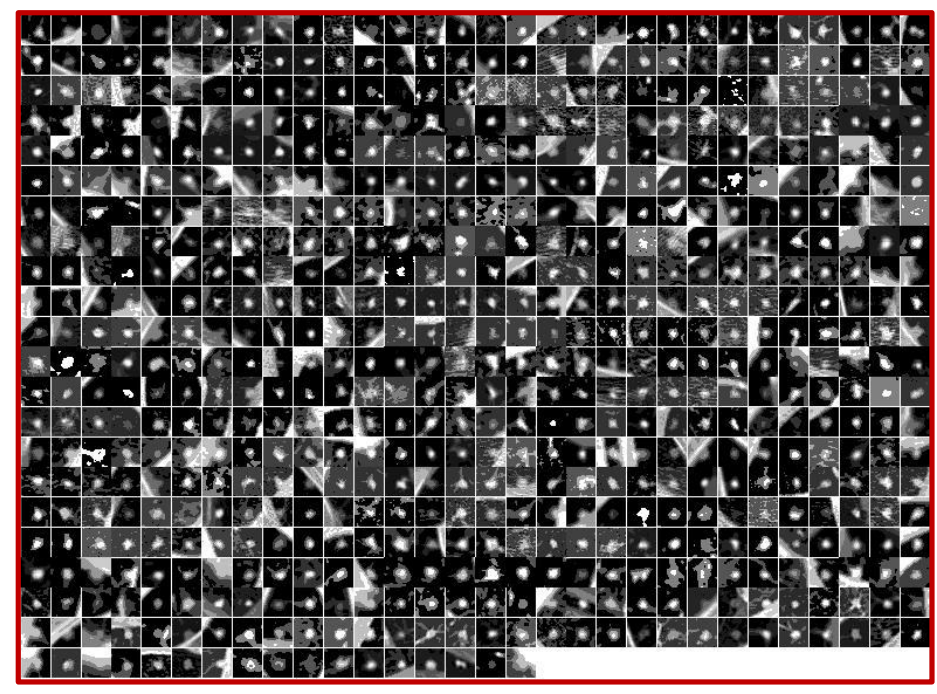

Figure 18: Sample of lung nodules at $10 \mathrm{~mm}$ or less in diameter, provided by the LIDC. This figure is meant to illustrate the large variation in nodule shape and appearance that is evident even in small populations. 
Figure 18 illustrates these factors with an ensemble of small lung nodules taken from the LIDC dataset.

\subsection{Nodule Modeling and Detection}

The goal of the data-driven approach is to capture texture and shape information from real-world nodules. This framework is based on the application of Active Appearance Modeling (AAM) and Active Shape Modeling (ASM). For application in image processing, each of these modeling approaches requires an annotated ensemble of images representing variations in the appearance and shape of the object which is to be modeled. Thus, as described before, the larger the population of nodules to pull from; the more accurate the resulting models. As proposed in [20], once a database of nodules has been acquired, a sub-database consisting of a minimum of 24 nodules per type are used to generate mean data-driven nodule templates (i.e. representations or models) to be used for candidate nodule detection. The sub-database of nodules are annotated to highlight the basic geometric and structural features of the nodules. Then a Procrustes based AAM approach is used to co-register the nodules and obtain a mean representation per type that captures the major features in terms of both shape and texture information. The data-

driven (non-parametric) approach to nodule modeling is discussed in detail in [8], and the framework presented is adopted in this thesis. 

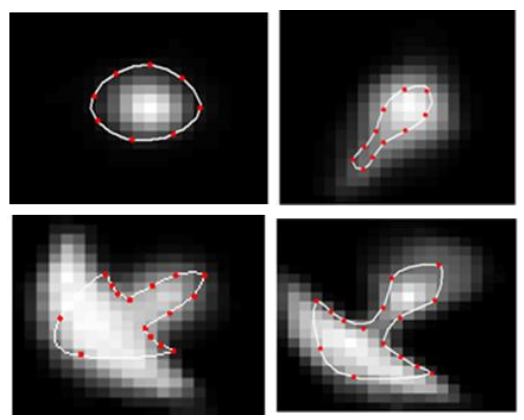

Figure 19: AAM models produced from an ensemble of 24 nodules taken from the ELCAP database. (Top Left) Well-Circumscribed, (Top Right) Vascularized, (Bottom Left) Juxta-Pleural, and (Bottom Right) Pleural-Tail.

Using nodule templates (models) from AAM and ASM, candidate nodules can be detected in CT scans using a template matching scheme. With the use of all four nodule templates, the system can detect nodules located at or close to the pleural surface, nodules embedded in vasculature and solitary nodules isolated in the lung tissue. Each template is independently swept across the lung tissue regions (obtained from lung tissue segmentation) in a raster fashion. At each new location, a region of interest is extracted from the original image, and a distance measure is computed between the current template and this region. By inspection of the nodule models in Figure 19, this method will return poor results due to nodule orientation. Thus, the proposed scheme iteratively rotates the nodule templates prior to the subsequent search. The system allows the user to specify how many rotations to account for due to timing constraints. Algorithmically, the search scheme is as follows: 
For each Nodule Template, $T_{N}: N=1,2,3,4$ (Well, Juxta, Tail, and Vascular)

For each Rotation, $R_{N}: 0 \rightarrow 360^{\circ}$

1.) Rotate $T_{N}$ by $R_{N}$ to obtain $T_{N}$,

2.) Raster across region containing lung tissue, $I(i, j)$. For each $i, j$ :

a. Crop a region of original image, $I_{C}$, the same size as $T_{N}$

b. Calculate some distance measure, $\delta$, between $T_{N}$ ' and $I_{C}$

c. If $\delta>$ threshold (set by user), Label $I(i, j)$ as a detected nodule

\section{End}

\section{End}

Table 3: General overview of the nodule detection algorithm used in the proposed system.

The detection scheme above returns an array of distance measures for each template at each rotation and the system handles up to (optional) 36 discrete rotations $-0 \rightarrow 360^{\circ}$ in $10^{\circ}$ increments. Thus, at a maximum, the process of sweeping a nodule template across the lung tissue in one slice occurs 36 times per template, totaling 144 passes per slice as shown in Figure 20. Assuming image resolution to be $512 \times 512$ and slices per scan to be approximately $250-350$, the process of detection can quickly become cumbersome. Thus, this detection procedure is sensitive to computation time and choice of distance measure. 


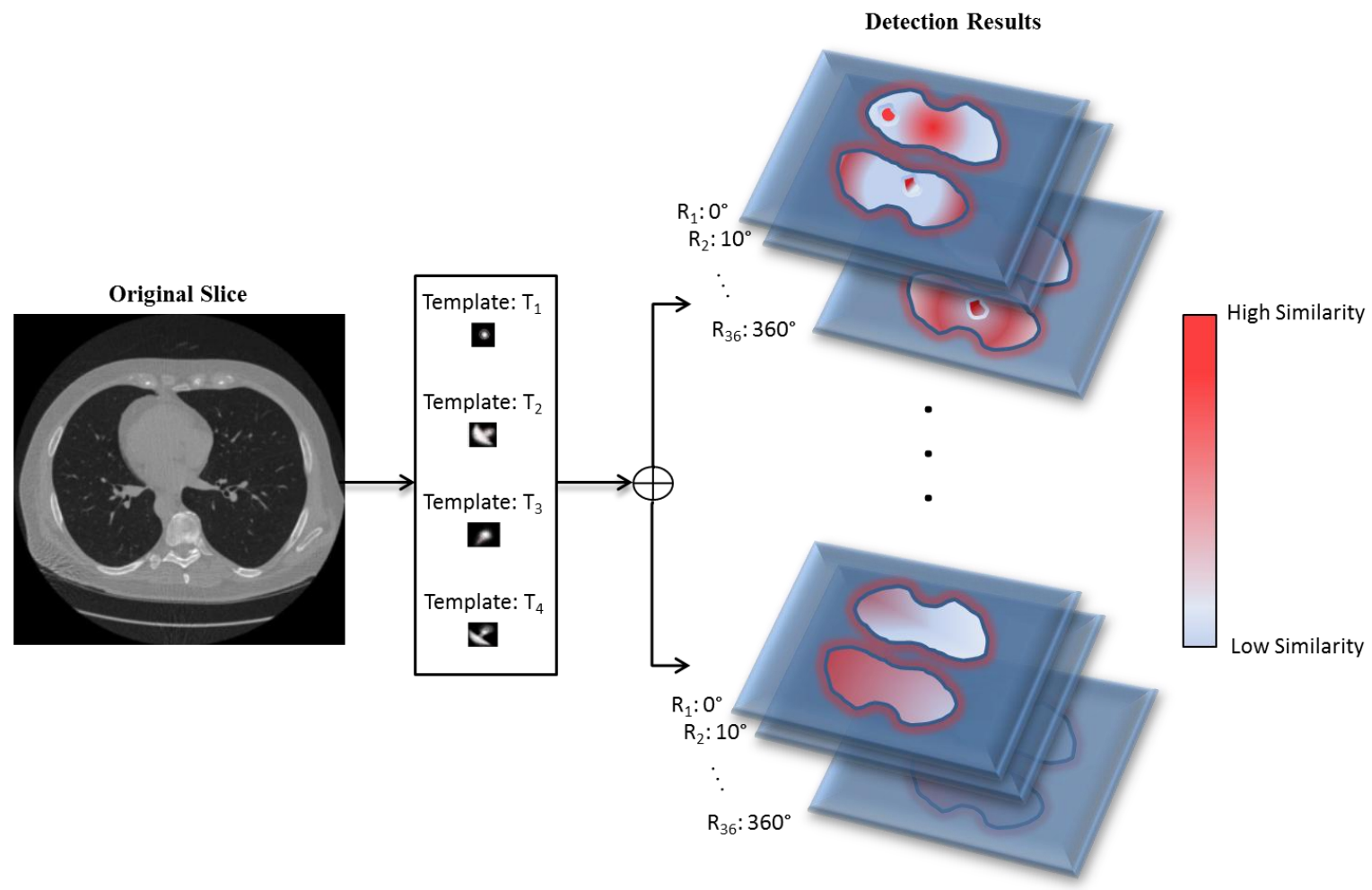

Figure 20: Illustration of the nodule detection scheme carried out on one slice, using each of the four templates at 36 rotations a piece. The process results in a stack of images corresponding to varying levels of similarity to one of the following templates at a given rotation.

Chapter 6 discusses optimizing computation time in detail using parallel programming techniques. The following section overviews distance measures historically used in this work as well as those which have been chosen to be viable for the CAD system.

\subsection{Distance Measures}

By definition, distance is a metric to describe how far apart two entities are. With respect to pattern recognition, distance may be cast as a similarity measure between two 
signals, i.e. images. A robust distance measure is needed to efficiently measure the similarity between a nodule template and a given region of lung tissue. In the previous work in [8] the following distance measures were tested for the application of nodule detection:

\begin{tabular}{|c|c|}
\hline $\begin{array}{l}\text { Normalized Cross } \\
\text { Correlation (NCC) }\end{array}$ & $\mathrm{NCC}=\frac{\sum_{(i, j) \in W} I_{t}(i, j) * I_{i}(x+i, y+j)}{\sqrt[2]{\sum_{(i, j) \in W} I_{t}^{2}(i, j) * \sum_{(i, j) \in W} I_{i}^{2}(x+i, y+j)}}$ \\
\hline $\begin{array}{l}\text { Zero-mean } \\
\text { Normalized Cross } \\
\text { Correlation (ZNCC) }\end{array}$ & $\mathrm{ZNCC}=\frac{\sum_{(i, j) \in W}\left(I_{t}(i, j)-\bar{I}_{t}(i, j)\right) *\left(I_{i}(x+i, y+j)-\bar{I}_{l}(x+i, y+j)\right)}{\sqrt[2]{\sum_{(i, j) \in W}\left(I_{t}(i, j)-\bar{I}_{t}(i, j)\right)^{2} * \sum_{(i, j) \in W}\left(I_{i}(x+i, y+j)-\bar{I}_{l}(x+i, y+j)\right)^{2}}}$ \\
\hline $\begin{array}{l}\text { Sum of Squared } \\
\text { Distance (SSD) }\end{array}$ & $\operatorname{SSD}=\sum_{(i, j) \in W}\left(I_{t}(i, j)-I_{i}(x+i, y+j)\right)^{2}$ \\
\hline $\begin{array}{l}\text { Zero-mean Sum of } \\
\text { Squared Distance } \\
\text { (ZSSD) }\end{array}$ & $\mathrm{ZSSD}=\sum_{(i, j) \in W}\left(I_{t}(i, j)-\bar{I}_{t}(i, j)-I_{i}(x+i, y+j)+\bar{I}_{l}(x+i, y+j)\right)^{2}$ \\
\hline $\begin{array}{l}\text { Locally scaled Sum of } \\
\text { Squared Differences } \\
\text { (LSSD) }\end{array}$ & $\operatorname{LSSD}=\sum_{(i, j) \in W}\left(I_{t}(i, j)-\frac{\bar{I}_{t}(i, j)}{\bar{I}_{\imath}(x+i, y+j)} I_{i}(x+i, y+j)\right)^{2}$ \\
\hline $\begin{array}{l}\text { Sum of Absolute } \\
\text { Differences (SAD) }\end{array}$ & $\mathrm{SAD}=\sum_{(i, j) \in W}\left|I_{t}(i, j)-I_{i}(x+i, y+j)\right|$ \\
\hline $\begin{array}{l}\text { Zero-Mean Sum of } \\
\text { Absolute Differences } \\
\text { (ZSAD) }\end{array}$ & $\mathrm{ZSAD}=\sum_{(i, j) \in W}\left|I_{t}(i, j)-\bar{I}_{t}(i, j)-I_{i}(x+i, y+j)+\bar{I}_{l}(x+i, y+j)\right|$ \\
\hline $\begin{array}{l}\text { Locally scaled Sum of } \\
\text { Absolute Differences } \\
\text { (LSAD) }\end{array}$ & $\mathrm{LSAD}=\sum_{(i, j) \in W}\left|I_{t}(i, j)-\frac{\bar{I}_{t}(i, j)}{\overline{I_{l}}(x+i, y+j)} I_{i}(x+i, y+j)\right|$ \\
\hline
\end{tabular}




\begin{tabular}{|l|l|}
\hline $\begin{array}{l}\text { Sum of Hamming } \\
\text { Distances (SHD) }\end{array}$ & SHD $=\sum_{(i, j) \in W} I_{t}(i, j)$ bitwise XOR $I_{i}(x+i, y+j)$ \\
\hline
\end{tabular}

Table 4: Distance measures considered in previous data-driven detection scheme. $I_{t}(i, j)$ represents the template, $I_{i}(x, y)$ is the image slice or input image, $W$ is the region of interest on which the similarity measure is evaluated, $\bar{I}_{t}($.$) is the mean of the template image, and \bar{I}_{l}($.$) Is the mean of$ the input image slice.

These distance measures have been exhaustively tested in [49] however no individual metric has proved superior. [8] found the LSSD to be inadequate for nodule detection. This narrows the criteria of selecting a metric for the CAD system down to one factor: computation time. In the spirit of engineering, the EMGU library (extension of OpenCV to $\mathrm{C \# )} \mathrm{provides} \mathrm{extremely} \mathrm{fast} \mathrm{implementations} \mathrm{of} \mathrm{the} \mathrm{following} \mathrm{distance}$ measures: Cross-Correlation (CC), Normalized Cross-Correlation (NCC), Sum of Squared Distance (SD), Normalized Sum of Squared Distance (NSD), Correlation Coefficient (CF), and Normalized Correlation-Coefficient (NCF). A comparison of computation time for each of these distance measures is provided in Chapter $\mathbf{6}$.

\subsection{False Positive Reduction}

In medical diagnostics, two measures of a detection scheme are typically calculated to profile the effectiveness of a given procedure: sensitivity and specificity. To understand these metrics, we must first understand the basics of Type I and Type II errors in statistics. As an example, assume a new test has been created to diagnose patients with Cancer and two patients undergo such a test. Further assume that Patient A 
truly does have Cancer while Patient B does not. The performance of the new test can be determined by monitoring the following measures:

1.) If the test determines that Patient A, does in fact have Cancer, this is classified as a true positive (TP).

2.) If the test determines that Patient $B$ has Cancer, this is classified as a False Positive (FP). This is a Type I Error.

3.) If the test determines that Patient A does not have Cancer, this is classified as a False Negative (FN). This is a Type II Error.

4.) If the test determines that Patient B does not have Cancer, this is classified as a True Negative (TN).

With these measures we can define sensitivity and specificity:

$$
\begin{aligned}
& \text { Sensitivity }=\frac{T P s}{T P S+\mathrm{FNs}} \\
& \text { Specificty }=\frac{T N s}{T N S+F P S}
\end{aligned}
$$

Sensitivity provides insight into the new test's capability of detecting positive results, i.e. identifying those patients with Cancer. Specificity provides insight into the new test's capability to detect negative results, i.e. healthy patients.

In the case of automatic lung nodule detection — especially early detection — the probability of Type I error is relatively high, given low spatial support, small nodule diameter, nodule resemblance to other anatomies, noise etc. In medical imaging applications, one would clearly prefer a high false positive rate versus a high false 
negative rate. However, the effectiveness of a CAD detection scheme will be miniscule if the operating physician is forced to sift through a sea of false positive nodules. The clinical value of such a system is low. Further, even if a false positive exemplifies a healthy patient, it is still an error in detection and could lead to unnecessary and invasive biopsy.

In [8], the highest reported sensitivity is $86.94 \%$ using AAM templates created from the ELCAP database. Further, the average sensitivity for all non-parametric experiments with these AAM templates is only $81.17 \%$. This work employs both the Scale-Invariant Feature Transform (SIFT) and the Local Binary Pattern (LBP) for False Positive Reduction (FPR), however neither of these methods yield feasible computation time for use in a clinical CAD system - with runtimes exceeding two days for only one scan in MATLAB. The proposed CAD system handles FPR in a much more interactive fashion — allowing the physician to calibrate the system to his/her preferred intensity of FPR.

The detection phase of the system yields a stack of correlation arrays corresponding to multiple rotations of multiple templates as shown in Figure 20. Each element of this stack represents correlation results from the same input slice. Each element is summed and normalized to generate one total correlation array. The FPR in this system can now be broken into two components, as provided by the system: 1.) Coarse and 2.) Fine. Both represent thresholds which truncate the detection results. The Coarse Threshold immediately disregards all pixels below its value. The Fine Threshold allows the user to window a certain percentage of those pixels that remain after applying the Coarse Threshold. See Figure 21 below: 


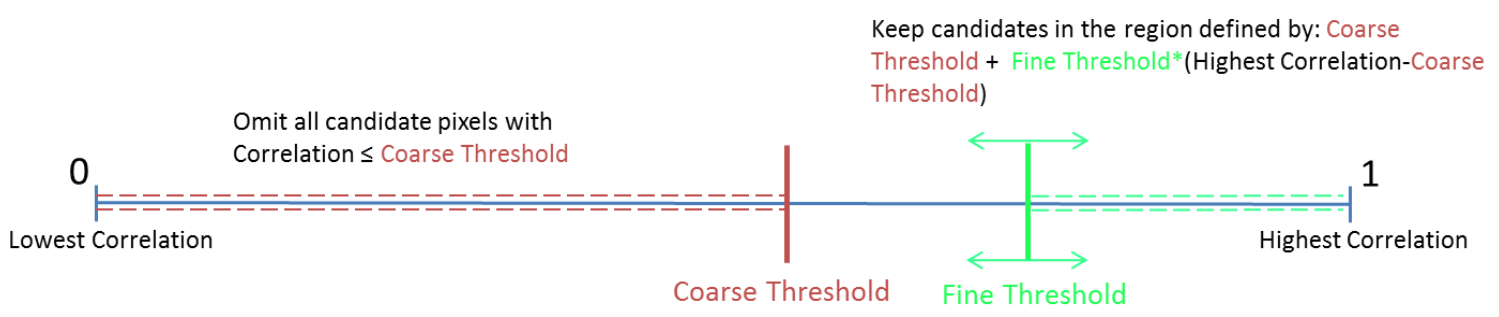

Figure 21: Illustration of False Positive Reduction for the case of the Normalized CrossCorrelation distance measure where correlations range between 0 and 1 . The Coarse Threshold rigidly truncates pixels and the Fine Threshold isolates the highest $x$ percent, depending on its value.

The implementation and usability of this FPR scheme is outlined in more detail in

\section{Chapter 6.}

\subsection{Tensor Modeling for Malignancy Classification}

As an aside, some extraneous work has been done in this thesis to mimic the tensor approach proposed by [17]. A tensor is an N-D array that describes relationships between its elements. A rank $n$ tensor in $m$-dimensional space is a mathematical object that has $n$ indices, $m^{n}$ components and obeys certain transformation rules. Thus a matrix is a $2^{\text {nd }}$-order tensor, a vector is a $1^{\text {st }}$-order tensor and a scalar is a $0^{\text {th }}$-order tensor. [17] proposed an N-D tensor framework as an extension to the Eigenface approach for the application of facial recognition. This work constructs a $6^{\text {th }}$-order face tensor using images of faces with the following dimensions: Illumination, Pose, Expression, Subject, Row Pixels, Column Pixels. In facial recognition literature, these dimensions are claimed 
to capture all variation in facial imaging. In this way, [17] enhances the EigenFace approach for recognition by adding dimensions.

The crux of this approach is building an N-D tensor. For the application of facial recognition above, a dataset had to be constructed consisting of 28 subjects, 5 poses each with 3 illuminations and 3 expressions - a total of 1260 images required to fill the tensor. To extend the AAM and ASM lung nodule models designed in [8] for the tensor approach requires an expansive dataset of nodules to choose from. Further, each element in the tensor must be filled, thus making the choice of dimensions a tradeoff between capturing significant variation and availability. Some preliminary work has been done with the LIDC dataset to adopt this approach for lung nodule modeling.

\subsubsection{Building a Data Tensor with Lung Nodules}

To build an adequate tensor, each nodule in the LIDC dataset was further classified based on the Kostis classification framework, as well whether the scan containing each nodule had been exposed to a contrasting agent. 4 dimensions were defined to maximize variability in the training population:

Dimension 1: Nodule Morphology (based on Kostis et. al framework)

1 - Well-circumscribed

2 - Juxta-Pleural

3 - Pleural Tail

4 - Vascular

Dimension 2: Illumination (Use of contrasting agent)

1 - No Contrasting Agent 


\section{2 - Contrasting Agent present}

Dimension 3: Nodule Margin - The radiologist's assessment of the sharpness of the nodule's margin (Provided by LIDC radiologists)

$$
1 \text { - Poor } \rightarrow 5 \text { - Sharp }
$$

Dimension 4: Nodule Sphericity - The radiologist's assessment of the roundness of the nodule.

$$
\begin{aligned}
& 1 \text { - Linear } \\
& 2 \\
& 3 \text { - Ovoid } \\
& 4 \\
& 5 \text { - Round }
\end{aligned}
$$

Each tensor element is a cropped DICOM image of a nodule which meets all intersecting criteria for the tensor seat. The size of the tensor (with the above dimensions) becomes 4 x $2 \times 5 \times 5 \times m \times n=200$ elts. of nodule images, $I_{m x n}$ (50 per nodule type).

\subsubsection{Solving for the Core Tensor, $Z$}

Given an image of a nodule, $I \in \boldsymbol{D}$ where $\boldsymbol{D}$ represents the data tensor, we need to align and resize such that $\forall I \in \boldsymbol{D}$ are of size $m \times n$. To do this, we deploy an alignment method in MATLAB taken from the AIT skin detection tutorial, [51]. Firstly, width, height, orientation and centroid of the binary region under consideration must be computed. For the LIDC, we define a binary nodule region as the area obtained by filling the contours provided by expert radiologists. Next we define a template nodule for each of the 4 classes: W-C, J-P, P-T and V. These template nodules can be the average nodule for each class. Then, each image is resized, rotated and its centroid placed on the 
centroid of the region in original grayscale image with only one region in it. We force a ground-truth size of $m x n$ for all four mean nodules that are generated. Given the governing equation:

$$
D=Z x_{1} \boldsymbol{U}_{\text {Class }} x_{2} \boldsymbol{U}_{\text {Contrast }} x_{3} \boldsymbol{U}_{\text {Margin }} x_{4} \boldsymbol{U}_{\text {Sphericity }} x_{5} \boldsymbol{U}_{\text {Pixels }}
$$

Were $x_{n}$ represents the mode- $n$ product

1. For $n=1, \ldots, N$, compute matrix $\boldsymbol{U}_{n}$ by computing the SVD of the flattened matrix $\boldsymbol{D}_{(n)}$ and setting $\boldsymbol{U}_{n}$ to be the left matrix of the SVD

2. Solve for the core tensor, $\mathbf{Z}$, as follows:

$$
Z=D x_{1} \boldsymbol{U}_{\text {Class }}{ }^{\boldsymbol{T}} x_{2} \boldsymbol{U}_{\text {Contrast }}{ }^{\boldsymbol{T}} x_{3} \boldsymbol{U}_{\text {Margin }}{ }^{\boldsymbol{T}} x_{4} \boldsymbol{U}_{\text {Sphericity }}{ }^{\boldsymbol{T}} x_{5} \boldsymbol{U}_{\text {Pixels }}{ }^{\boldsymbol{T}}
$$

A dataset of nodules to build a data tensor cannot be collected in same way as a dataset for faces. There is no controlled protocol for data collection of lung nodulesthey come as they come. Thus, the success in building a data tensor using the LIDC was an unexpected surprise. Using MATLAB code provided by [17], a sample of tensor reprojections taken from the Nodule Tensor are shown below in Figure 22:

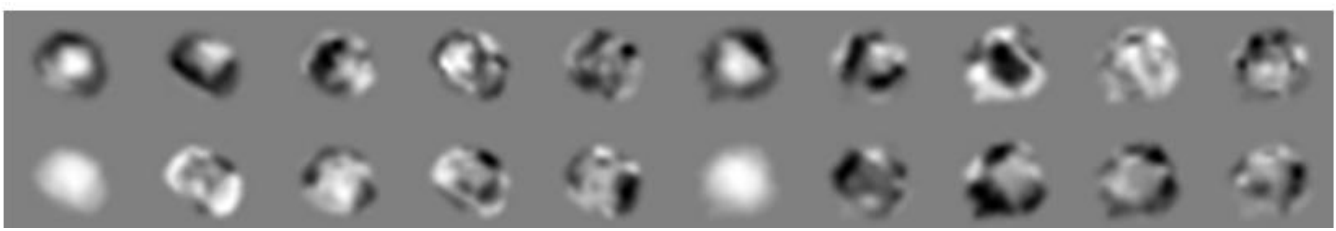

Figure 22: Sample of tensor re-projections obtained using the Tensor Toolbox provided by [17]. 


\section{Chapter 4: Nodule Segmentation}

\subsection{Overview, Motivations and Challenges}

Segmentation is a process that is defined in Chapter 2. As applied in medical imaging, segmentation is plagued by several challenges such as inhomogeneous anatomies, ambiguous boundaries, resolution, occlusion, noise, and low spatial support. In the case of lung nodule detection and diagnosis, these challenges are difficult for both CAD systems and radiologists themselves to overcome — especially low spatial support when the task is early detection (nodules roughly less than $3-5 \mathrm{~mm}$ in effective diameter). These difficulties allow for continuing research in lung nodule segmentation.

The significance of segmenting lung nodules is validated by the notion that shape and growth can be good indicators of malignancy. The precise segmentation of lung nodules thus serves the purpose of computationally determining the exact size of the nodule as well as retrieving some shape metric, which may be used as predictors of malignancy. Shape is directly related to the morphology of a lung nodule, which is used in radiological practice as an indicator of malignancy — along with features such as size and morphology. A spiculated nodule has irregular shape, contrasting that of a smooth, round nodule, which appears more elliptical and without points of high curvature along the contour. Smooth, round nodules are more likely to be benign whereas spiculated nodules have a much higher likelihood of malignancy. This correlation between morphology and malignancy presents a need for the accurate extraction of exact nodule boundaries in current CAD systems aimed at lung nodule detection. The solution of this 
segmentation problem is motivated by providing some shape metric, such as mean curvature, as quantification of nodule morphology — thus, a more exact indicator of malignancy. Further, the accurate extraction of a tight nodule contour allows for a more accurate size metric for growth tracking as well as an extension into 3-D nodule visualization. Some challenges regarding nodule segmentation include: Small nodule size, varying occurrence w.r.t. anatomical location with lung cavity, nodule merging with other anatomies such as vasculature and pleural surface, noise and in-homogeneities generated from the imaging process, and a lack of strong edges exhibited by some lung nodules.

\subsection{Preprocessing}

In this stage of the pipeline, we wish to accurately extract the nodule from the CT slice. With a precise contour around the nodule, we can quantify shape and texture information strictly from the nodule itself-ignoring the surrounding tissue. From our detection step, we are assuming with high confidence that we have a pixel belonging to the domain of pixels that we classify as 'nodule'. The initial step in nodule extraction is auto-cropping. The goal is to obtain a rough, but tight, crop box around the nodule to narrow the effort of a segmentation routine, which will perform extraction. To handle this, we employ a very simple and effective region-growing algorithm. From the detected seed point, four lines (centered about the seed point of considerable length) are swept in a raster fashion in each cardinal direction away from the seep point. Each growing line propagates or terminates based on the frequency information of the pixels 
lying on it. Given an empirically determined threshold, we terminate growth based on the weighted sum of the mean intensity and the mean intensity gradient of each line, independently:

$$
\begin{gathered}
\text { if } \alpha \mu_{I}^{n}+\beta \mu_{\nabla I}^{n}>T \text {, grow } \\
\text { else, terminate } \\
\text { where } \alpha, \beta \text { : empirically determined weights } \\
\mu_{I}^{n}: \text { the mean intensity along the } n^{\text {th }} \text { line } \\
\mu_{\nabla I}^{n}: \text { the mean intensity gradient along the } n^{\text {th }} \text { line } \\
\text { T: empirically determined threshold }
\end{gathered}
$$

Once each propagating front has terminated, we obtain a crop box by default using their intersection. In this fashion, we may narrow the domain with which our segmentation routine must operate and with some accepted crop box surrounding the nodule, we can more effectively deploy a segmentation routine to extract the exact boundary of the nodule.

\subsection{Level Sets with Shape Priors}

In the method proposed by Abdelmunim et al. [32] the Level-Set Segmentation framework described in Chapter $\mathbf{2}$ is augmented by embedding a prior shape model into the energy functional. This recasts the process of segmentation as a procedure closer to registration, in which prior shape models generated from the four categories proposed by Kostis' et al. are registered with candidate nodule contours. This framework does not 
lend itself to implementation within the CAD system because it requires prior knowledge of which of the four nodule categories the candidate nodule belongs in order to initialize the correct shape model. In this stage of the pipeline, this is not feasible. Regardless, for the sake of completeness, some sample results of this procedure are shown below in

Figure 23 and Figure 24.
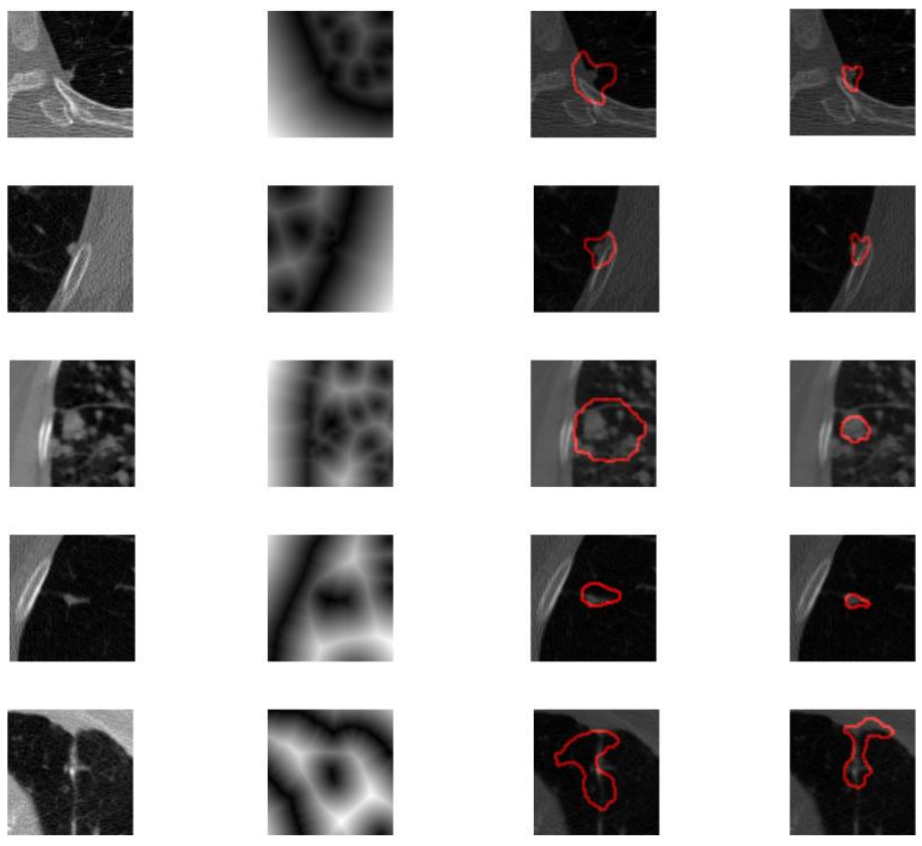

Nodule Image

$\varphi_{g}$

Initial $\varphi_{p}$

Final $\varphi_{p}$

Figure 23: Illustration of the level set algorithm as applied to nodule segmentation. (Left Column) Initial nodule crop-box. (Left Middle Column) (Right Middle) Initial contour. (Right) Final nodule contour. 

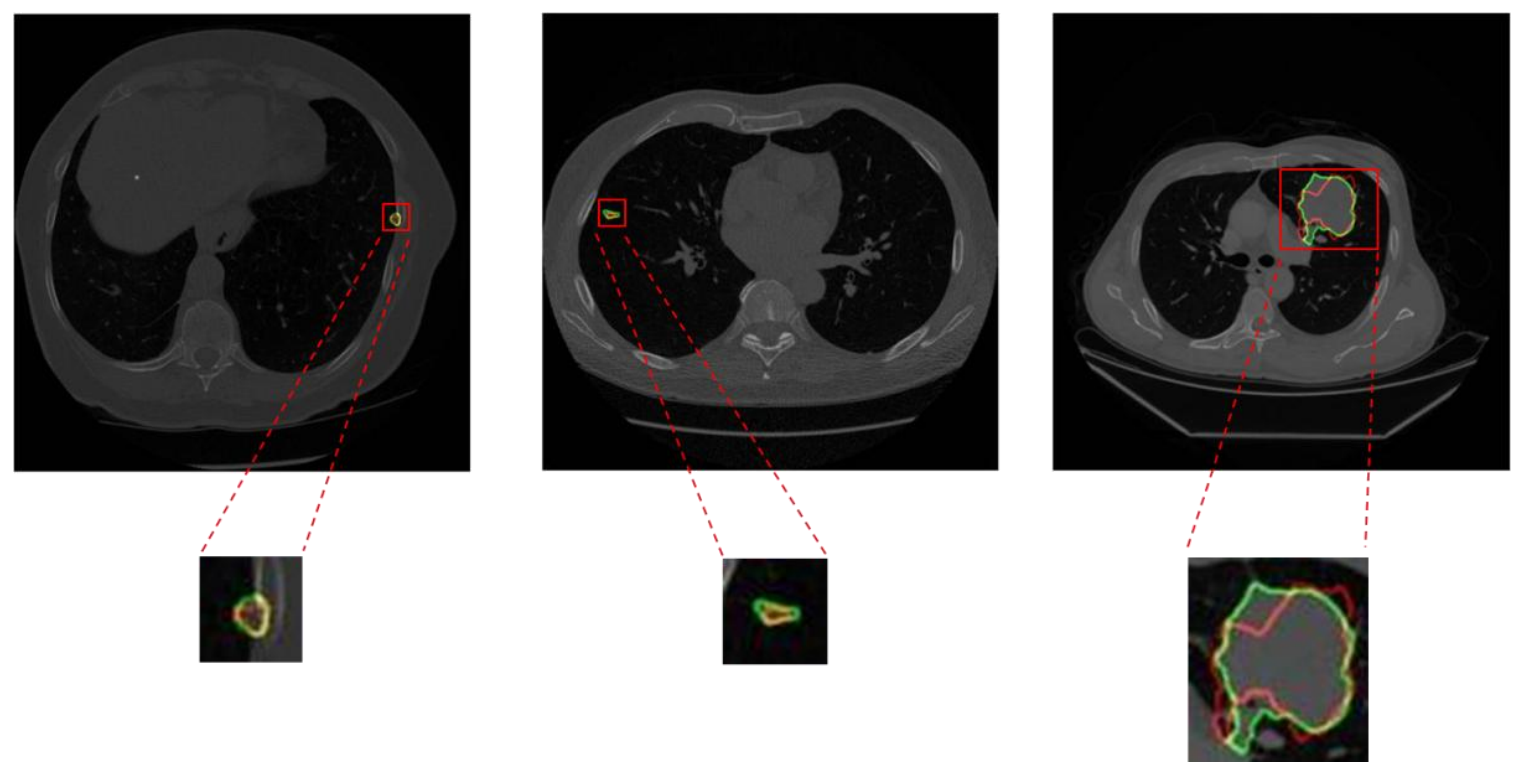

Figure 24: Sample results from the level set method driven with shape priors. This method is relatively successful but requires prior knowledge of the nodule type (according to 1 of the 4 categories outlined in this thesis).

\subsection{Expectation Maximization for Nodule Segmentation}

The method here is the same as in Chapter 2. As such, this strictly intensitybased segmentation scheme is not as sensitive to weak edges, low spatial support, noise etc. However, the resulting nodule segmentation will have to undergo morphological post-processing in order to obtain homogeneous, segmented regions.

\subsection{Variational Ellipse}

The use of variational segmentation methods (such as Level-Sets) has been discussed in Chapter 2 and is implemented above in Section 4.2. However, these methods aim at extracting a contour based on intensity and gradient information. For 
solitary nodules (Well-Circumscribed), these methods will succeed but for Vascularized, Pleural-Tail and Juxta-Pleural, these methods will generally include extraneous anatomies such as the pleural wall or vasculature due to homogeneity and strong edges. It should be noted that the definition of a nodule does not include surrounding anatomies. When a physician measures nodule characteristics, such as size, they only account for the "head" of the nodule. Thus, a new variational solution was proposed in [32], which simplifies the variational approach to accommodate only the nodule head. The solution forces an elliptical contour to deform until convergence, in an attempt to isolate the region of the nodule candidate that best resembles the elliptical appearance of a nodule head.

This process aims to compute a transformation that warps a candidate shape $(\alpha)$ to a target shape $(\beta)$. The source and target shapes are represented by Signed-Distance Maps $\phi_{\alpha}$ and $\phi_{\beta}$. We further assume scale, rotation and translation elements: $\boldsymbol{S}=$ $\operatorname{diag}\left(s_{x}, s_{y}\right), \theta$ (associated with a general rotation matrix $\left.\mathbf{R}\right)$ and $\boldsymbol{T}=\left[T_{x}, T_{y}\right]^{T}$. The signed distance map can be expressed in terms of its projections in $x$ and $y$ directions as: $\mathbf{d}=\left[d_{x}, d_{y}\right]^{T}$ at any point in either shape. Applying a global transformation, $\mathbf{A}$ on $\phi_{\alpha}$ results in $\mathbf{d}_{\alpha}^{\prime}=\mathbf{R S} \mathbf{d}_{\alpha}$. Thus, the magnitude is defined as: $\phi^{\prime}{ }_{\alpha}=\left\|\mathbf{S} \mathbf{d}_{\alpha}\right\|$ and the constraint $\phi^{\prime}{ }_{\alpha} \leq \max \left(s_{x}, s_{y}\right) \phi$ arises. The difference between the warped shape and target shape can be formulated as:

$$
r(\mathbf{X})=\left\|\mathbf{R S d}_{\alpha}(\mathbf{X})\right\|-\phi_{\beta}(\mathbf{A})
$$

Which yields:

$$
E_{1}=\int_{\Omega} \delta^{\prime}{ }_{\epsilon}\left(\phi_{\alpha}, \phi\right) r^{2} d \Omega
$$


where $\delta^{\prime}{ }_{\epsilon}$ reduces the complexity of the problem and $\epsilon$ is the size of the narrow band around the contour. The given measure $r$ satisfies $r \leq s \phi_{\alpha}(\mathbf{X})-\phi_{\beta}(\mathbf{A})$, where $\mathrm{s}=$ $\max \left(s_{x}, s_{y}\right)$. Thus, if $E \leq E_{1}$, the following energy function results;

$$
E=\int_{\Omega} \delta^{\prime}{ }_{\epsilon}\left(\phi_{\alpha}, \phi_{\beta}\right)\left(s \phi_{\alpha}(\mathbf{X})-\phi_{\beta}(\mathbf{A})\right)^{2} d \Omega
$$

The parameters $\left\{s_{x}, s_{y}, \theta, T_{x}, T_{y}\right\}$ are required to minimize the energy functional $E$. A more in-depth derivation can be found in [32].

The following algorithm results from the above formulation:

1. Construct the initial prior shape ellipse and its shape model representation $\emptyset_{p}$.

2. Compute the intensity segmentation region representation $\emptyset_{g}$ using Level Set evolution. Iterate until the function converges.

3. Initialize the transformation parameters to $s_{x}=1, s_{y}=1$ and $\theta=0$. Assuming that the nodule center is known which initializes the translation parameters $t_{x}$ and $t_{y}$.

4. Solve the gradient descent approach to minimize the energy. Parameters converge to their steady state values and hence the final boundaries of the ellipse are computed.

5. (Optional) Threshold the region inside the ellipse to accurately mark the nodule pixels. The resulting region may under-go a median filter smoothing step to remove noisy pixels.

Table 5: Variational Ellipse algorithm used for nodule segmentation. This method uses the LevelSet evolution of a forced elliptical boundary in order to isolate the nodule head from the surrounding anatomies as in the cases of Vascularized, Juxta-Pleural and Pleural-Tail nodules.

The advantages of this approach are: 1.) no required, prior model 2.) independent of initialization 3.) independent of nodule size 4.) independent of nodule location and 5.) independent of nodule category. 


\subsection{HEAT}

This algorithm was developed by the author and is as follows. Given a 2-D signal, $I(x)$, where $x$ is some node, suppose we have $n$ classes within $I(x)$. For our purposes, let $I(x)$ be the crop box around the nodule. Given $I(x)$, we can easily extract the histogram $h(x)$, which upon inspection, generally possesses few "natural thresholds", which we are defining as zero-crossings in $h(x)$.
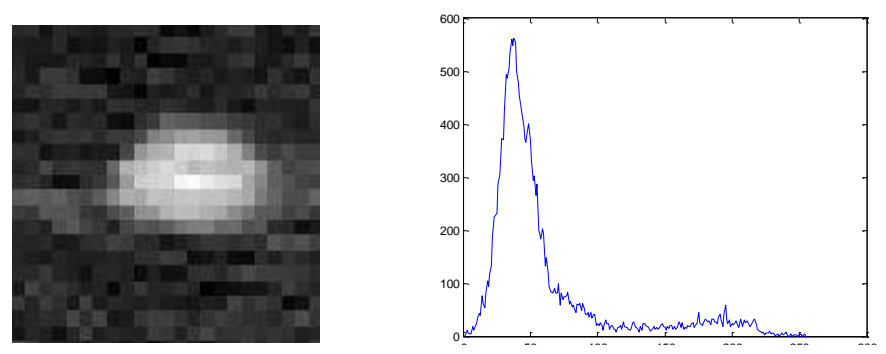

Figure 25: (Left) Solitary nodule detected from a low-dose CT scan. (Right) Histogram of detected nodule.

In reference to the histogram above in Figure 25, many techniques, such as ExpectationMaximization, rely on the inference of optimum thresholding based on information directly exhibited by the signal itself, i.e. gradient information, or a-priori information to isolate individual classes and thus segment the nodule. Further, some thresholding techniques require prior knowledge about the number of classes in the signal or even a prior probability density function to which the current histogram can be fitted to. The Heat algorithm pursues an ideal weighting function, $w(x)$, such that upon the process of generating a new signal: $J(x)=I(x) w(x)$, we promote "natural thresholds" in the updated histogram i.e. obtaining the appropriate number of isolated regions in the histogram of $J(x)$, where isolated regions are defined as bandwidths with non-zero area 
in the histogram with natural boundaries defined by occurrences of zero frequency. The goal is to find $w(x)$ such that $|n-r|$ is min, where $\mathrm{n}$ is the true number of classes in $I(x)$ and $\mathrm{r}$ is the number of isolated regions in $\operatorname{hist}(J(x))$. The proposed solution to this problem is to let $w(x)=h(x)$, where $h(x)$ is the histogram of $I(x)$, and assumed to be the probability density function of the image

The results from employing this solution for an image of a lung nodule appear as follows:
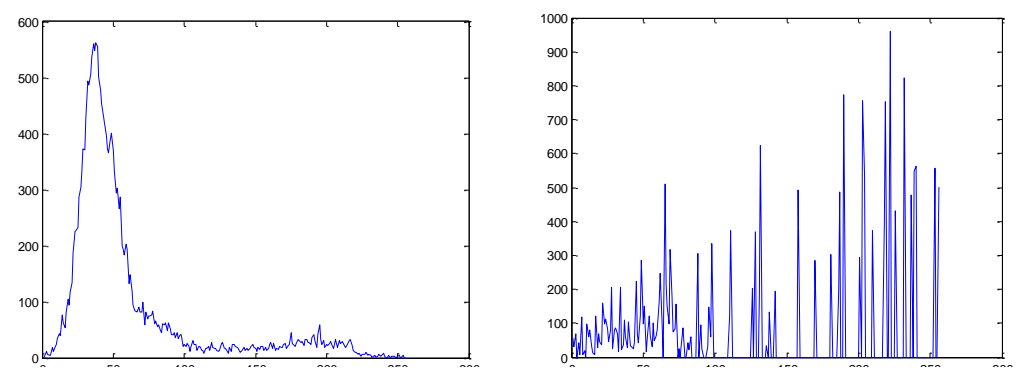

Figure 26: (Left) Original histogram of $I(x)$, the nodule in Figure 1. (Right) The normalized histogram of $J(x)$, the resulting in the multiplication of $I(x)$ with its own probability density.

By inspection of the updated histogram in Figure 26, one can immediately see the large number of natural thresholds generated by weighting the original image in such a fashion. It should be noted that the histogram of the updated image has been normalized back from $0-255$. In this way, we are performing a very simple compression and in doing so, we are binning infrequent pixels to a single class of pixels in the updated image. This class represents pixels of little interest in the image, i.e. noisy pixels. 


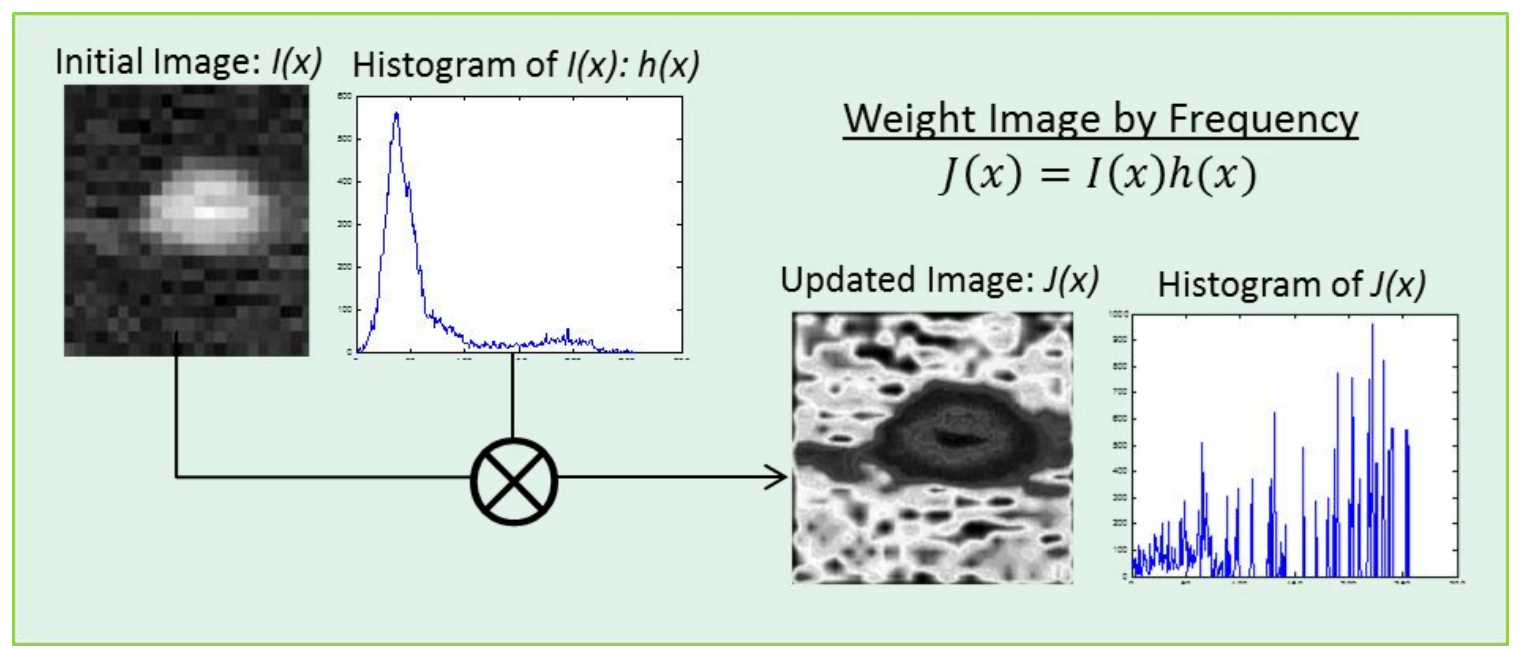

Figure 27: The initial step of the HEAT algorithm. The updated image $J(x)$ becomes the image to be segmented.

Using a simple image labeling procedure, the input image is partitioned into classes based on the natural thresholds computed. The output of applying the Heat algorithm is shown in the labeled image in Figure 28.
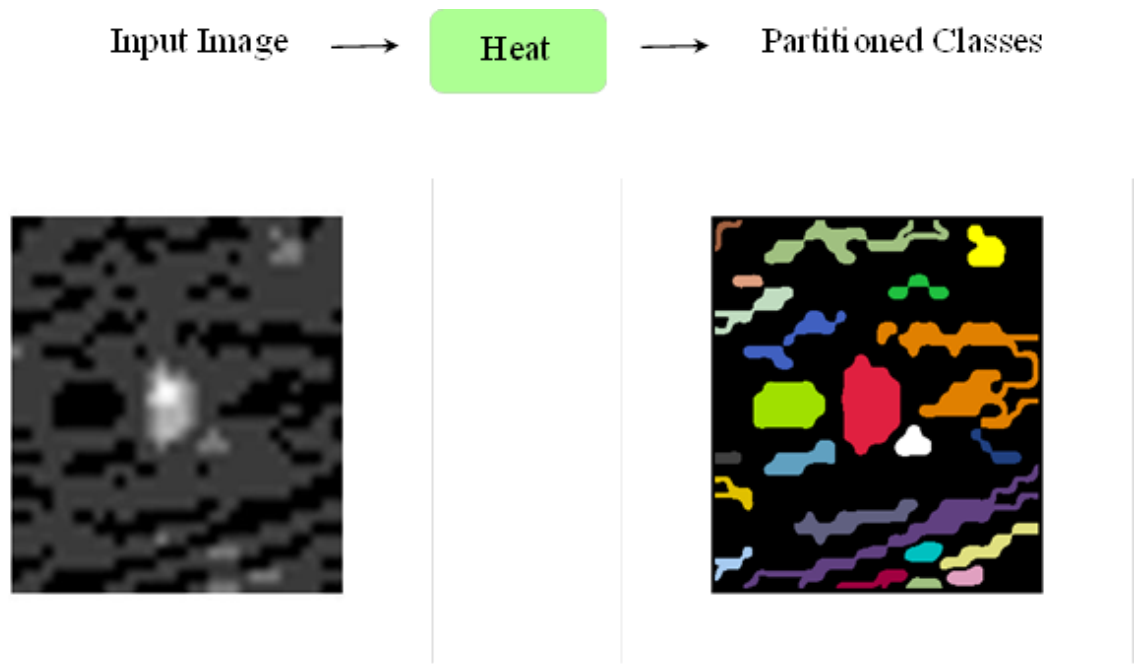

Figure 28: Results from inputting a crop box of a lung nodule into the HEAT algorithm. Individual classes are shown in different colors on the right. 
To obtain the final nodule segmentation, the Heat algorithm needs a single seed point belonging to isolate the class of interest from the labeled image. In this framework, this seed will come from pixels corresponding to a high correlation in the detection phase of the system.

For the purposes of lung nodule segmentation, this algorithm is sufficient and extremely fast. It requires no prior knowledge of nodule classification, shape, or appearance. The disadvantage of this method is the inclusion of anatomies attached to the nodule, such as vasculature or the pleural wall. The Heat generally classifies connected components together unless their intensity greatly differs.

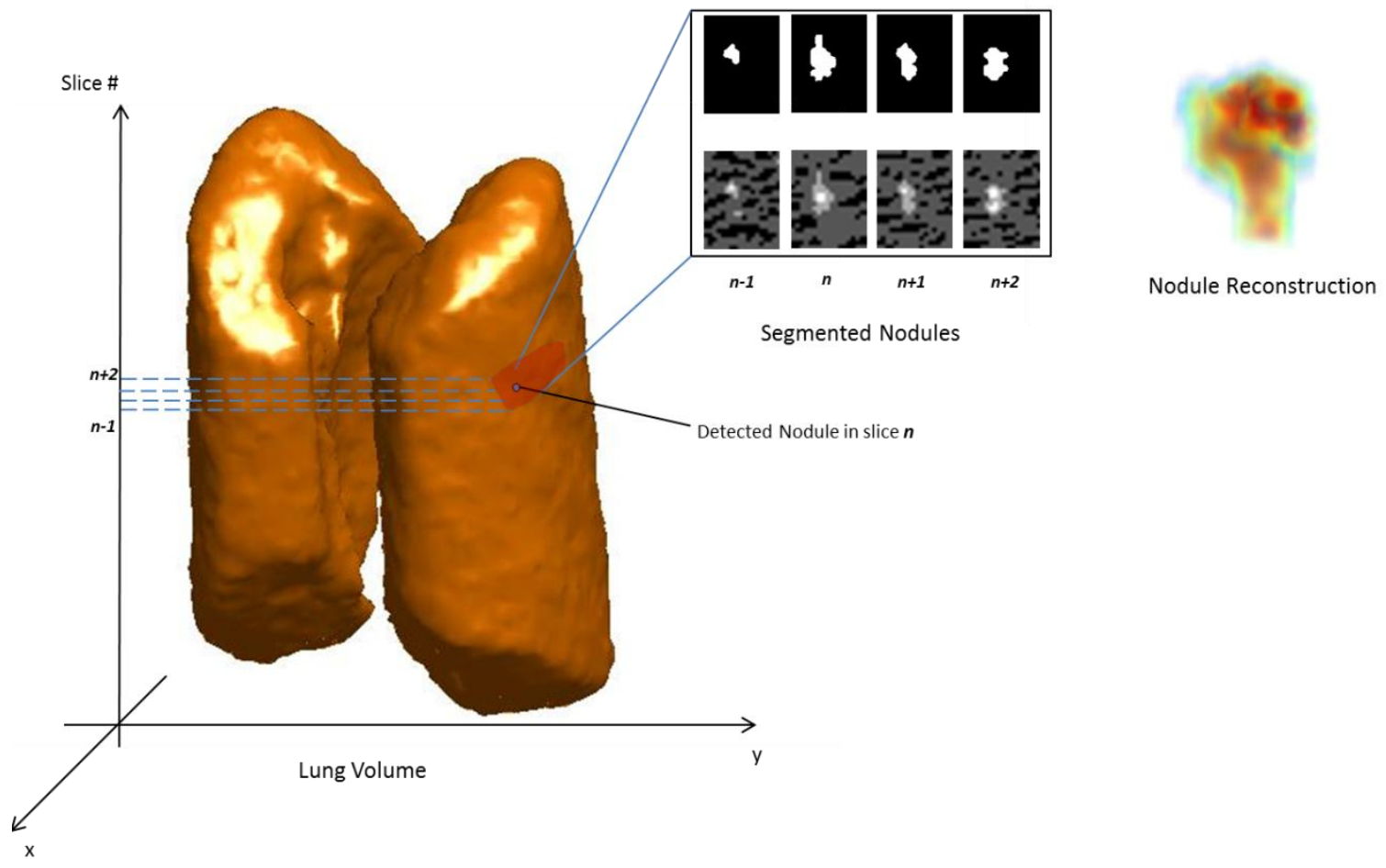

Figure 29: 3D nodule reconstruction obtained from segmenting a nodule appearing in four slices using the HEAT algorithm. It should also be noted that the HEAT algorithm was used to obtain the segmented tissue allowing for the lung cavity reconstruction shown on the left. 


\subsection{Preliminary Results}

To evaluate performance, this thesis makes use of the Lung Image Database Consortium (LIDC) [10] nodule database, which provides expert segmentations (defined as 'regions of interest') in the database for each nodule in the database, carried out independently by at least four radiologists. These expert segmentations allow for a direct comparison between the proposed algorithm and up to four professional opinions on the exact nodule boundary. Segmentation results are visualized for several methods below.

\begin{tabular}{|c|c|c|c|c|c|c|}
\hline & Original & EM & Variational & $\begin{array}{c}\text { Shape } \\
\text { Variational }\end{array}$ & $\begin{array}{c}\text { Shape } \\
\text { Variational } \\
+ \text { EM }\end{array}$ & $\begin{array}{c}\text { Graph Cuts } \\
\text { (Mask) }\end{array}$ \\
\hline $\begin{array}{c}\text { Radiologist } \\
1\end{array}$ & & & & & & \\
\hline $\begin{array}{c}\text { Radiologist } \\
2\end{array}$ & & & & & & \\
\hline $\begin{array}{c}\text { Radiologist } \\
3\end{array}$ & & & & & & \\
\hline $\begin{array}{c}\text { Radiologist } \\
4\end{array}$ & & & & & & \\
\hline
\end{tabular}

Figure 30: Comparison of LIDC nodule segmentation using the described methods. The scan from which the nodule was extracted had been exposed to a contrasting agent (Shown above). The cropped nodule region used for segmentation was taken from the manual outlines provided by the

LIDC, thus the listing for multiple radiologists. This also contests to algorithmic consistency. 
LIDC Results-Nodule 3 (Post)

\begin{tabular}{|c|c|c|c|c|c|c|}
\hline & Original & EM & Variational & $\begin{array}{c}\text { Shape } \\
\text { Variational }\end{array}$ & $\begin{array}{c}\text { Shape } \\
\text { Variational } \\
+ \text { EM }\end{array}$ & $\begin{array}{c}\text { Graph Cuts } \\
\text { (Mask) }\end{array}$ \\
\hline $\begin{array}{c}\text { Radiologist } \\
1\end{array}$ & & & & & & \\
\hline$\underset{2}{\text { Radiologist }}$ & & & & & & \\
\hline$\underset{3}{\text { Radiologist }}$ & & & & & & \\
\hline $\begin{array}{c}\text { Radiologist } \\
4\end{array}$ & & & & & & \\
\hline
\end{tabular}

LIDC Results-Nodule 55 (Pre)

\begin{tabular}{|c|c|c|c|c|c|c|}
\hline & Original & EM & Variational & $\begin{array}{c}\text { Shape } \\
\text { Variational }\end{array}$ & $\begin{array}{c}\text { Shape } \\
\text { Variational } \\
\text { + EM }\end{array}$ & $\begin{array}{c}\text { Graph Cuts } \\
\text { (Mask) }\end{array}$ \\
\hline $\begin{array}{c}\text { Radiologist } \\
1\end{array}$ & & & & & & \\
\hline $\begin{array}{c}\text { Radiologist } \\
2\end{array}$ & & & & & & \\
\hline $\begin{array}{c}\text { Radiologist } \\
3\end{array}$ & & & & & & \\
\hline $\begin{array}{c}\text { Radiologist } \\
4\end{array}$ & 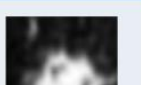 & 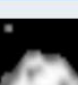 & I & & & \\
\hline
\end{tabular}


LIDC Results-Nodule 211 (Pre)

\begin{tabular}{|c|c|c|c|c|c|c|}
\hline & Original & EM & Variational & $\begin{array}{c}\text { Shape } \\
\text { Variational }\end{array}$ & $\begin{array}{c}\text { Shape } \\
\text { Variational } \\
+ \text { EM }\end{array}$ & $\begin{array}{c}\text { Graph Cuts } \\
\text { (Mask) }\end{array}$ \\
\hline $\begin{array}{c}\text { Radiologist } \\
1\end{array}$ & & & & & & \\
\hline $\begin{array}{c}\text { Radiologist } \\
2\end{array}$ & & & & & & \\
\hline $\begin{array}{c}\text { Radiologist } \\
3\end{array}$ & & & & & & \\
\hline
\end{tabular}

LIDC Results-Nodule 56 (Pre)

\begin{tabular}{|c|c|c|c|c|c|c|}
\hline & Original & EM & Variational & $\begin{array}{c}\text { Shape } \\
\text { Variational }\end{array}$ & $\begin{array}{c}\text { Shape } \\
\text { Variational } \\
+ \text { EM }\end{array}$ & $\begin{array}{c}\text { Graph Cuts } \\
\text { (Mask) }\end{array}$ \\
\hline $\begin{array}{c}\text { Radiologist } \\
1\end{array}$ & & & & & & \\
\hline
\end{tabular}

LIDC Results-Nodule 1212 (Pre)

\begin{tabular}{|c|c|c|c|c|c|c|}
\hline & Original & EM & Variational & $\begin{array}{c}\text { Shape } \\
\text { Variational }\end{array}$ & $\begin{array}{c}\text { Shape } \\
\text { Variational } \\
+ \text { EM }\end{array}$ & $\begin{array}{c}\text { Graph Cuts } \\
\text { (Mask) }\end{array}$ \\
\hline $\begin{array}{c}\text { Radiologist } \\
1\end{array}$ & & & & & & \\
\hline
\end{tabular}

LIDC Results-Nodule 2110(Pre)

\begin{tabular}{|c|c|c|c|c|c|}
\hline & Original & EM & Variational & $\begin{array}{c}\text { Shape } \\
\text { Variational }\end{array}$ & $\begin{array}{c}\text { Shape } \\
\text { Variational } \\
+ \text { EM }\end{array}$ \\
\hline $\begin{array}{c}\text { Radiologist } \\
1\end{array}$ & & & & & \\
(Mask) & & \\
\hline
\end{tabular}

Figure 31: Comparison on five more LIDC nodules. Nodules from scans with (Post) and without (Pre) contrasting agents are considered. 
To further evaluate the performance of the following, some sample Receiver Operating Characteristic (ROC) curves were calculated. ROC curves are created by plotting the change in true positive rate versus the change in false positive rate while any given system parameter is varied. The true positive rate is synonymous with the Sensitivity and the false positive rate is equivalent to 1-Specificity. For the purposes of segmentation, we can define the measures of TP, FP, TN, and FN as follows:

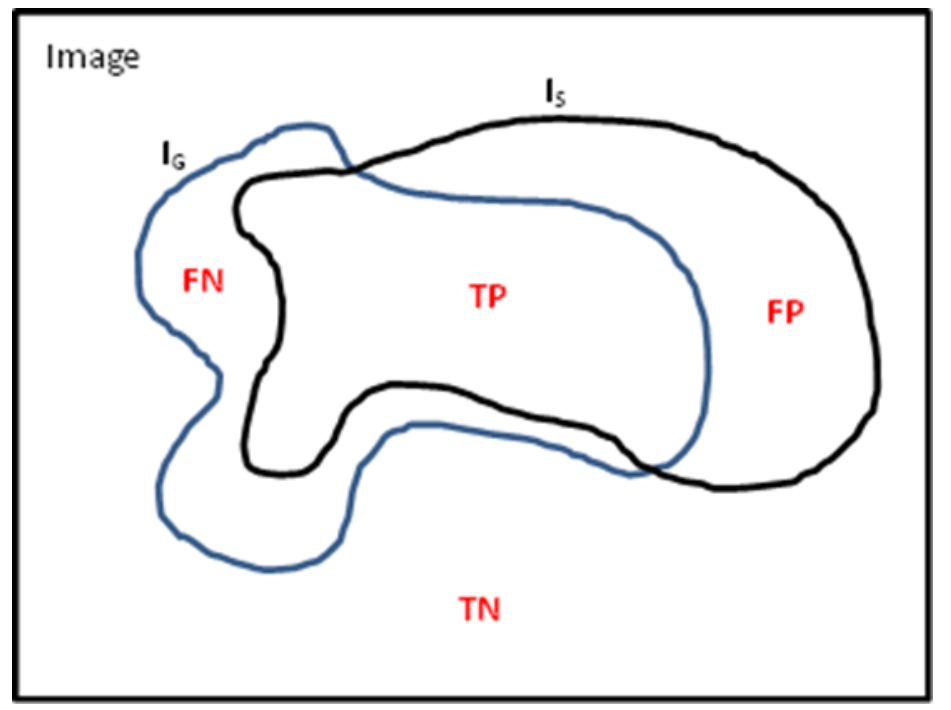

Figure 32: $I_{s}$ refers to the segmentation results, $I_{G}$ refers to the ground truth segmentation. TP refers to the true positive region, FP refers to the false positive region, $T N$ refers to the true negative region and $\mathrm{FN}$ refers to the false negative region.

Let $I_{S}$ be the nodule segmentation results for a given method and let $I_{G}$ be the ground truth nodule segmentation obtained from an expert. Thus:

$$
\begin{aligned}
& \text { TP region }=I_{S} \cap I_{G} \\
& \text { FP region }=I_{S} \backslash I_{G}=I_{G}{ }^{C} \cap I_{S}
\end{aligned}
$$




$$
\begin{aligned}
& \text { FN region }=I_{G} \backslash I_{S}=I_{S}{ }^{C} \cap I_{G} \\
& \text { TN region }=\left(I_{S} \cup I_{G}\right)^{C}
\end{aligned}
$$

Using these measures, a scatter plot of ordered pairs, corresponding to individual nodule segmentations can be obtained, and thus averaging over all instances will yield ROC curves such as those shown in Figure 33 and Figure 34. These figures illustrate performance for the standalone EM algorithm as well as the Variational Ellipse augmented by the EM algorithm. In [8], it has been determined that the Variational Ellipse combined with the EM algorithm provides the best nodule segmentation performance. This trend is also apparent in the following figures.

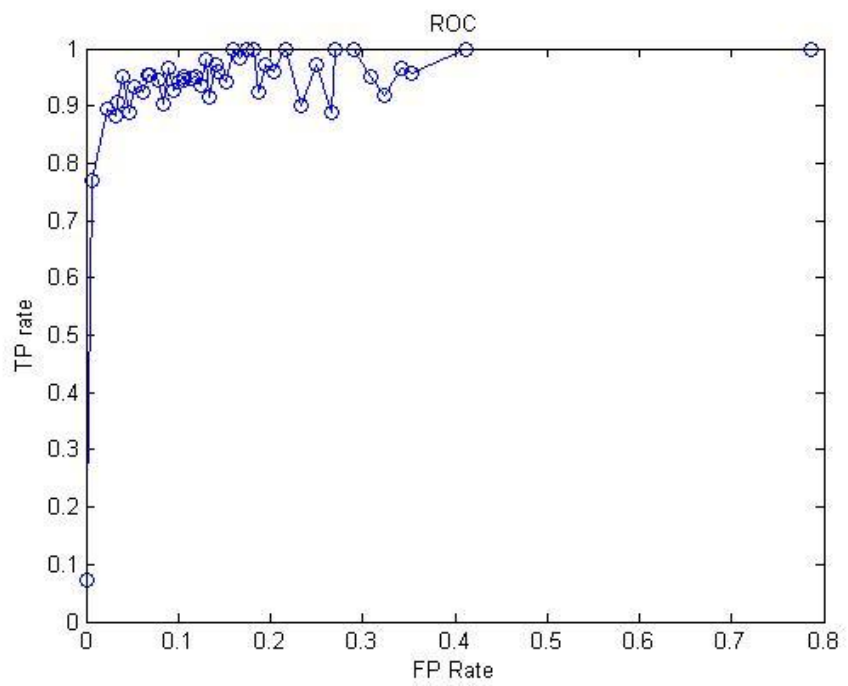

Figure 33: Receiver operating characteristic curve for segmentation of LIDC nodules using the Expectation Maximization algorithm. The EM algorithm is strictly intensity based. 


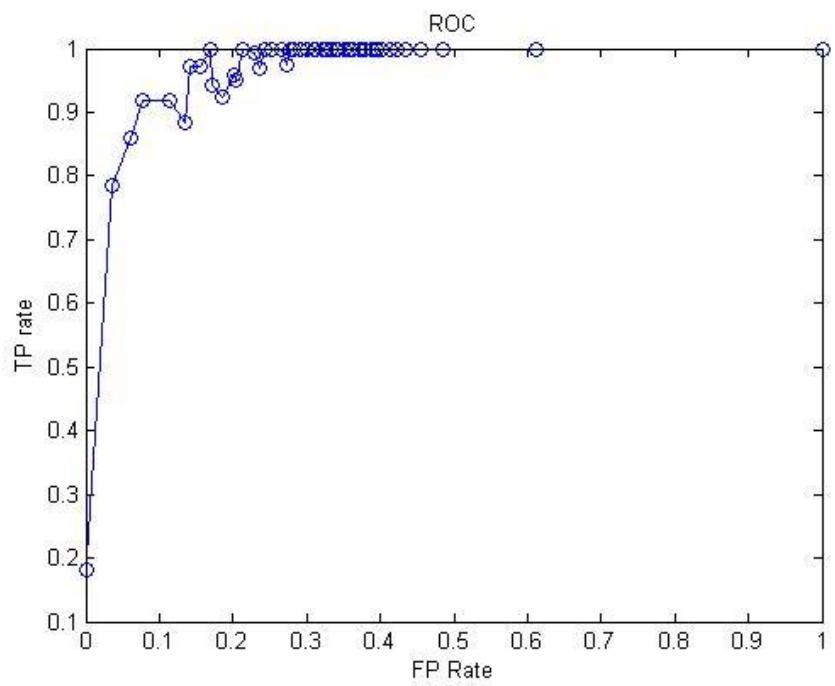

Figure 34: Receiver operating characteristic curve for segmentation of LIDC nodules using the Variational Ellipse followed by the Expectation Maximization algorithm. This combinatorial algorithm is more robust than the previous approaches as shown by the quicker saturation of the ROC curve.

The curves here do differ from traditional ROC curves, in that they do not saturate as smoothly. This jagged saturation pattern is a result of averaging the TP Rate for each FP rate. Further, segmentation results were obtained whether one or all of the participating radiologists provided an expert nodule contour, thus introducing error.

The results of accurately segmenting nodules allow for the accurate tracking of growth for reoccurring patients. Figure 35 shows the 3-D growth of a nodule taken from a patient belonging to the Jewish Hospital database. Using the Variational Ellipse combined with the EM algorithm, an accurate volume metric was obtained for the nodule appearing in scans conducted over a 2.5 year period. 


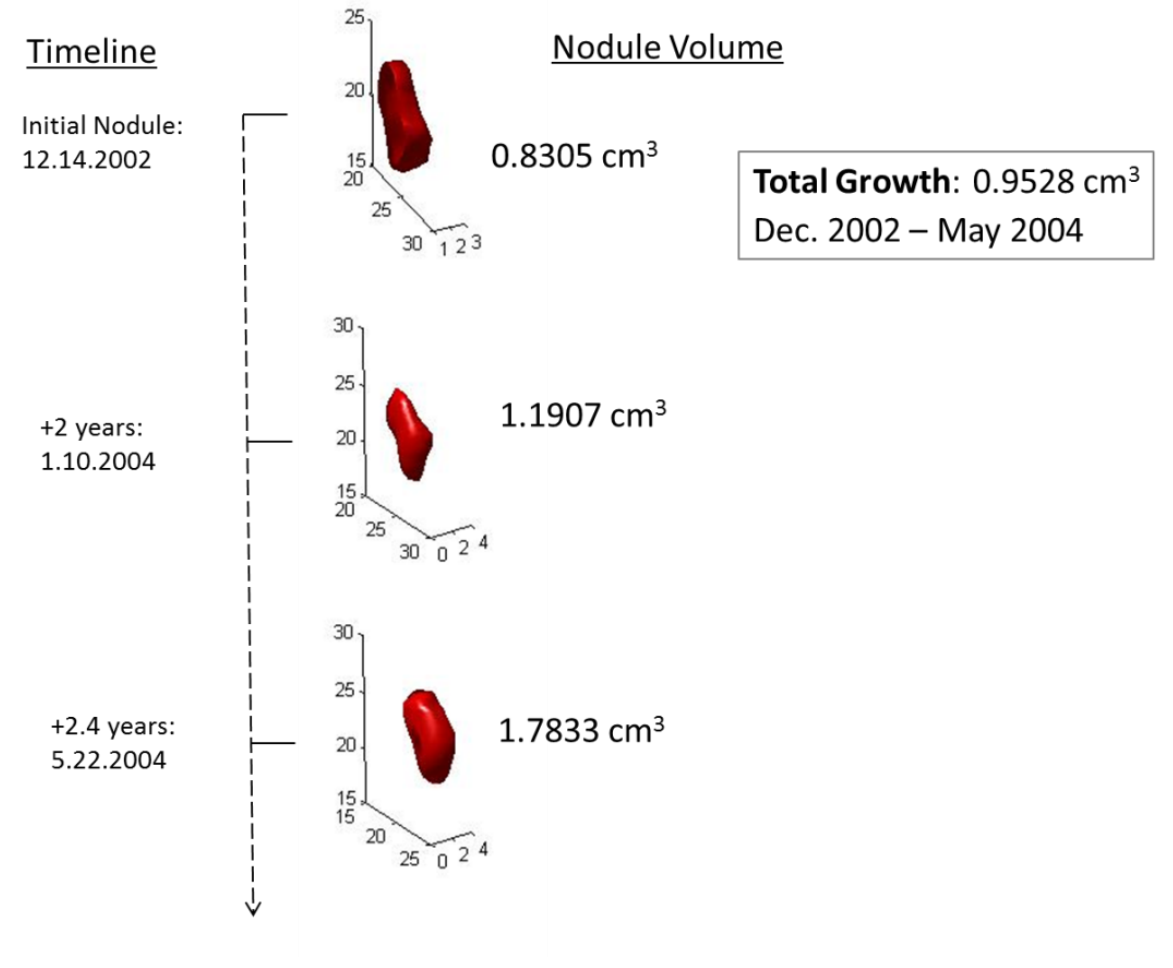

Figure 35: Radiologists correlate nodule size (volume) with malignancy; moreover, nodule growth rate and doubling rate. If our system can temporally track nodule growth rate then we can effectively mimic/aid radiologists in diagnosis. Further, our growth analysis is in 3-D with supplementary visualization.

The option of growth tracking is a very significant contribution made by the Nodule Segmentation branch of the overall pipeline. It allows the physician a more precise understanding of the shape, texture, and metric features of detected lung nodules. 


\section{Chapter 5: Nodule Classification}

\subsection{Overview, Motivations and Challenges}

From an engineering perspective, the task of classifying detected lung nodules is an exceptionally well-suited problem for the exhaustive employment of machine learning techniques - both in the arenas of feature extraction/representation and classification. This part of the pipeline is expansive and variable in the current literature. The purpose of the classification step in this framework is to categorize nodules into four primary categories: Well-Circumscribed, Juxta-Pleural, Pleural-Tail, and Vascularized as discussed above. First, this chapter will overview and evaluate some current nodule classification techniques.

In [13], type-2 Fuzzy Logic Systems (FLS) are applied for the binary classification of lung nodules into two categories: Nodule and Non-Nodule. The purpose of this classification is to introduce a false-positive reduction step, which follows the groups prior work in nodule detection. The contribution of [13] is an automatic approach for learning and tuning Gaussian interval type-2 membership functions (IT2MFs) for application to multi-dimensional pattern classification problems. FLS require the estimation of parameters to model a footprint of uncertainty of a Gaussian interval type-2 fuzzy set. Genetic algorithms are used to estimate the parameters of the footprint of uncertainty. 
This method outperforms this group's prior classification accuracy by $30 \%$ achieving a peak accuracy of $95 \%$ however, nodules are significantly larger than those in this thesis and the dataset used has only 81 nodules. This greatly compromises the universality of this classification scheme. Regardless, the feature descriptors used for profiling nodules are as follows:

\begin{tabular}{|c|c|c|}
\hline No. & Feature & Definition \\
\hline 1 & Volume & The size of the nodule \\
\hline 2 & Diameter & The effective nodule diameter \\
\hline 3 & Sphericity & The degree of being spherical \\
\hline 4 & $\begin{array}{l}\text { Mean HU- } \\
\text { Spherical }\end{array}$ & $\begin{array}{l}\text { The mean } \mathrm{HU} \text { of the spherical part of the } \\
\text { nodule }\end{array}$ \\
\hline 5 & Elongation & The degree of elongation of the nodule \\
\hline 6 & $\begin{array}{l}\text { Mean HU- } \\
\text { Elongated }\end{array}$ & $\begin{array}{l}\text { The mean } \mathrm{HU} \text { of the elongated part of the } \\
\text { nodule }\end{array}$ \\
\hline 7 & Distance & $\begin{array}{l}\text { The distance from the nodule to the thoracic } \\
\text { wall }\end{array}$ \\
\hline
\end{tabular}

Table 6: Features used in [reference Hosseini et. al] for the classification of candidate nodules into two categories: Nodule or Non-Nodule for the purposes of reducing false positives from a prior detection scheme.

In order to employ a FL Classifier, this paper outlines classification rules than were developed based on mimicking clinical practice. The rules listed outline criteria that indicate a high probability of the candidate belonging to the class of Nodule. These rules are listed below: 
1.) If Volume is high and Sphericity is high

2.) If Volume is high and Distance is mid

3.) If Volume is high and Diameter is high

4.) If Sphericity is high and Volume is mid

5.) If Sphericity is high, Volume is high and Distance is high

6.) If Sphericity is high and Elongation is not low

7.) If Sphericity is high and MeanHU_Spherical is high

8.) If Elongation is not low

9.) If Sphericity is not low

10.) If Distance is high and Diameter is high

11.) If Elongation is high, Sphericity is high and Diameter is mid

12.) If Elongation is high and Diameter is not mid

13.) If Distance is high

14.) If MeanHU_Elongated is not high and MeanHU_Spherical is not low

Using the above rules, a given nodule candidate is thus classified as Nodule or NonNodule. The primary contribution by this work is the use of FLS to emulate the process of nodule classification by an expert using a simple schematic of rules.

In an alternative approach a CAD system is designed and evaluated for detecting lung nodules, [11]. The contribution of [11] is the addition and validation of features for lung nodule detection/classification and determining the optimum performance between the following classifiers: Fisher Linear Discriminant, Gaussian Bayes Linear Classifier and Quadratic Classifier. This work was tested on an unnamed, publicly available dataset 
consisting of 154 nodules; 100 malignant and 54 benign, and trained on 181 nodules from a different database.

The feature descriptors used are extensive in this work. The features used are categorized as follows: 9 geometric, 18 intensity-based, 17 gradient-based. It is important to note that not all features are used for each classifier. The classifiers used are empirically selected based on optimizing the classifier performance. The Fisher Linear Discriminant uses 46 features, the Gaussian Bayes Linear Classifier uses 42 features, the Quadratic Classifier uses 15 features. The numbers of features used by each classifier are chosen to maximize a sequential forward selection objective function. Testing is done using 10-fold cross-validation technique. Further experiments are carried based different stratifications of the data. The data is stratified by:

1.) Size: $<10 \mathrm{~mm}=$ Small, $10 \mathrm{~mm}<\mathrm{x}<20 \mathrm{~mm}=$ Medium and $>20 \mathrm{~mm}=$ Large

2.) Pathology: Malignant or Benign

3.) Subtlety: Radiologists have annotated nodules as one of the following: extremely subtle, very subtle, subtle, relatively obvious and obvious.

FROCs are generated by thresholding classifier posterior probabilities and scoring the results. The optimum classifier in these experiments has shown to be Fisher Linear Discriminant and further the highest performance w.r.t size was exhibited by large nodules $(>20 \mathrm{~mm}$ in effective diameter).

In [12], a previously developed CAD system is enhanced by using a classification scheme as a technique for false-positive reduction. This work uses the Asymmetric Adaboost as a classifier and it is theoretically compared with symmetric Adaboost. The 
Symmetric Adaboost classifier penalizes all errors equally. For example, the cost for a False Negative is equal with the cost for a False Positive, with respect the Loss Function below:

$$
L_{S}(i)= \begin{cases}1 & C\left(x_{i}\right) \neq y_{i} \\ 0 & \text { otherwise }\end{cases}
$$

Where $C\left(x_{i}\right)$ is the class designated to some data, $y_{i}$ is the true class of the data.

The goal of Adaboost is to minimize the error function:

$$
E_{S}=\sum_{i} L_{S}
$$

On the other hand, the Asymmetric Adaboost allows for weighted penalization of False Positives and False Negatives separately. For a CAD system, the symmetric penalization of errors is not ideal. For a medical application, we wish to penalize False Negatives far more than False Positives. Consider the new Loss Function:

$$
L_{A}(i)=\left\{\begin{array}{c}
\sqrt{k} \text { for } y_{i}=1 C_{i}=-1 \\
1 / \sqrt{k} \text { for } y_{i}=-1 C_{i}=1 \\
0 \text { otherwise }
\end{array}\right.
$$

Where $k=\frac{N_{n}}{N_{P}} A, \mathrm{~N}_{\mathrm{n}}=\#$ Non-Nodules, $\mathrm{N}_{\mathrm{P}}=$ \# Nodules, A: asymmetry parameter (scalar) 
In this way, the Asymmetric Adaboost penalizes False Negatives more and thus corrects the boosting procedure.

The feature descriptors used belong to four different categories: Shape-based, Intensity-based, Covariance-Matrix-based and Hessian Matrix-based. The shape, intensity and covariance based features rely on the segmented nodule candidate while the Hessian features depend on a 10mm-cube neighborhood around the estimated candidate centroid. This framework was tested on database of 357 nodules with an overall system sensitivity of 74.3\%, with an average of 2.6 False Positives per slice. Experiments are carried out on scans with slice thicknesses of $1 \mathrm{~mm}$ and image resolutions of $0.416 \mathrm{~mm} /$ pix. Further, this work carries out detection and classification separately for two independent classes: Parenchymal (non-pleural) and Juxta-pleural. As compared to the Kostis' categorization, the Parenchymal class handles Well-Circumscribed and Vascularized nodules while the Juxta-pleural class handles Juxta-Pleural and Pleural-Tail nodules.

The number of Adaboost steps are empirically determined somewhere between 50-100 steps. The classifier learning takes 7 hours in MATLAB and the overall system sensitivity is $74.3 \%$ with an average of 2.6 False Positives/Slice.

\subsection{Preliminary Feature Descriptors and Classifiers}

Previous work in this area proposes two classic dimensionality reduction schemes, Princimpal Component Analysis (PCA) and Linear Discriminant Analysis 
(LDA). These techniques are used to extract the most relevant information from the eigen-images of the nodules. Beyond PCA and LDA, this framework has made use of two more modern feature extraction techniques: SIFT and LBP. The LBP in the Computer Vision Literature is a texture based feature descriptor that is invariant to monotonic changes in gray-scale and illumination resistant. Previously, this approach has used the extended LBP operator within a $(\mathrm{P}, \mathrm{R})$ neighborhood with only uniform patterns and is noted as $\mathrm{LBP}_{\mathrm{PR}}^{\mathrm{u} 2}$. The obtained LBP results are depicted from using both the original and gradient images, where Sobel filters $\left(h_{x}\right.$ and $\left.h_{y}\right)$ where used to generate the gradient magnitude image.

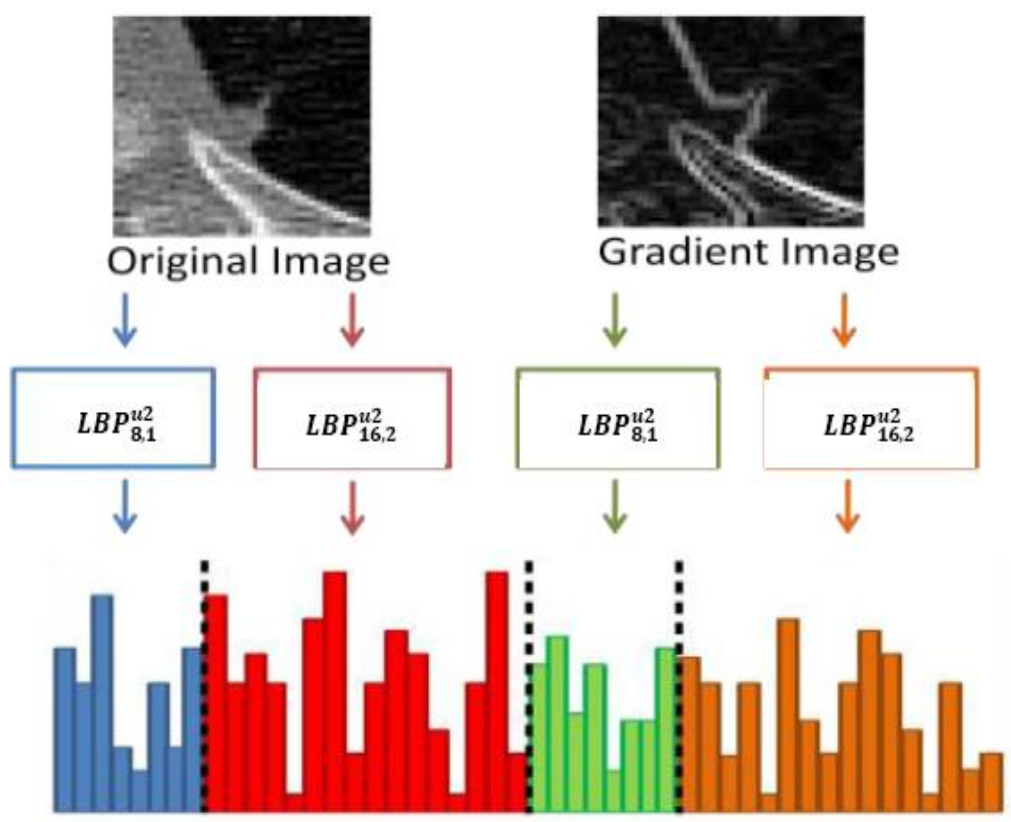

Figure 36: Block Diagram of generating the LBP for a juxta-pleural nodule. The equation for the above picture is: $\mathrm{LBP}_{8,1}^{\mathrm{u} 2}+\mathrm{LBP}_{16,2}^{\mathrm{u} 2}+\mathrm{LBP}_{8,1}^{\mathrm{u} 2}+\mathrm{LBP}_{16,2}^{\mathrm{u} 2}$ where the first two terms represent the original image and the last two terms represent the gradient image. 
The second feature descriptor that has been previously considered is the SIFT. SIFT consists of four main steps: (1) Scale-space peak selection; (2) Key-point localization; (3) Orientation assignment; and (4) Key-point descriptor. More on SIFT for this application can be found in [8]. The features discussed here exhibit many desirable properties such as scale and rotation invariance but they can be computationally expensive for the exhaustive application to every detected nodule in the system. The lack of nodule spatial support will also limit these descriptor's ability to extract feasible features - especially the SIFT, which fails on small images and requires resizing to perform. Previous work in this area also includes the use of simple classifiers such as the k-Nearest Neighbors (k-NN) approach to classify nodules with the above features. These methods may be included in the final CAD system for completeness, however for the sake of computation time and simplicity, the primary classification machinery in this system is based on the same principles used in the detection phase of the pipeline.

\subsection{Classification in the CAD system}

The proposed detection framework in Chapter $\mathbf{3}$ relies on data-driven modeling approaches proposed in [20]. Using multiple rotations, templates based on the four nodule categories discussed above are swept through a scan and correlations are obtained, which quantify candidate similarity to the current template. As such, the obvious extension for classifying nodules directly follows as a result of tabulating which template provided the highest correlation for any pixel deemed as a nodule! The classification scheme is as follows: 
Initialize $h=0$;

Iterate:

For each Nodule Template, $T_{N}: N=1,2,3,4$ (Well, Juxta, Tail, and Vascular)

For each Rotation, $R_{N}: 0 \rightarrow 360^{\circ}$

1.) Rotate $T_{N}$ by $R_{N}$ to obtain $T_{N}$,

2.) Crop a region around detected nodule, $I_{C}$, the same size as $T_{N}$

a. Calculate some distance measure, $\delta$, between $T_{N}$ ' and $I_{C}$

End

b. Store all $\delta$ values in $\boldsymbol{d}$

If $\max (\boldsymbol{d})>h: h=\max (\boldsymbol{d}) \&$ Nodule Class $=$ Nodule Template

End

Return Nodule Class

Table 7: Classification scheme implemented in the CAD system. This approach is a direct extension of the detection approach and acts to immediately classify a detected nodule using the same distance measures that detected the nodule from the raw scan.

The above approach can be deployed at the time of detection or during the later review of detection results. The advantage of this approach is the lack of need for cumbersome feature extraction, which after consuming computation time, may result in poor classification results due to low spatial support. This method is extremely fast and effective as illustrated in Chapter 6. 


\section{Chapter 6: A Functional User Interface}

\subsection{Overview}

The goal of this chapter is to outline the designed CAD system. The pipeline components described above theoretically validate the approaches implemented in the CAD system shown below. The system has been developed in the language of $\mathrm{C \#}$ and aims at collecting manually annotated nodule data from radiologists in a fashion that closely resembles clinical reading for the purpose of building a large dataset of lung nodules, while providing the user with the a second-opinion diagnosis based on the

pipeline described above. As stated earlier in this thesis, this CAD system is meant to be a diagnostic tool, not a replacement for an expert.

The motif in developing this system has been keeping each phase modular. The system has been engineered such that new approaches can be easily deployed in parallel with those that currently exist in the CAD system. This holds for each stage of the pipeline, Tissue Segmentation, Nodule Detection, and Nodule Classification. As such, any future enhancement to the pipeline is very straightforward to add, remove or update. The interface layout has been designed to mimic standard software for reading medical images such as OsirisX [52]. Most of the interface real-estate is reserved for viewing the slice of interest while toolboxes have been fixed on either side for quick use during reading. A screenshot of the interface is shown in Figure 37. 


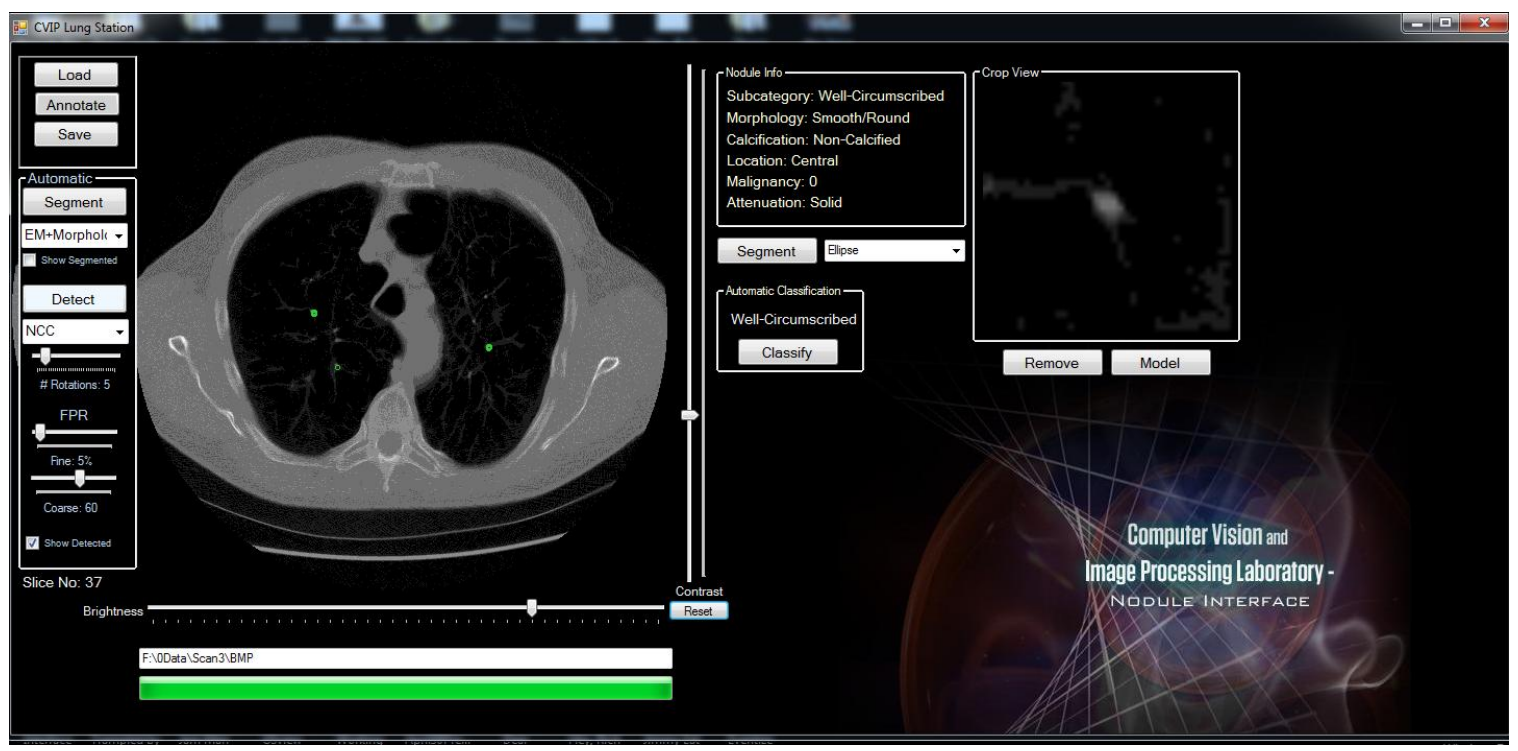

Figure 37: Screenshot of the designed interface. The interface is meant to be simple, fast and functional. Most of the interface consists of the slice view (Axial slice shown above), while the regions surrounding the slice view hold toolboxes which assist the user in annotating nodules. Note: the annotation shown in the right-hand pane of the interface is just for demonstration purposes and does not pertain to a real nodule.

A detailed schematic of the interface is provided in Appendix A.

\subsection{Data Acquisition}

\subsubsection{Manual Annotation}

As discussed in Chapter 1, one of the primary goals of the designed CAD system is collecting large of amounts of lung nodules, annotated by physicians, to build a database which will be used to enhance the nodule models discussed briefly in Chapter

3. The data collection scheme in this interface is as follows:

1.) Prompt the user to load a CT scan using a Load button. (Shown in Figure 38) 


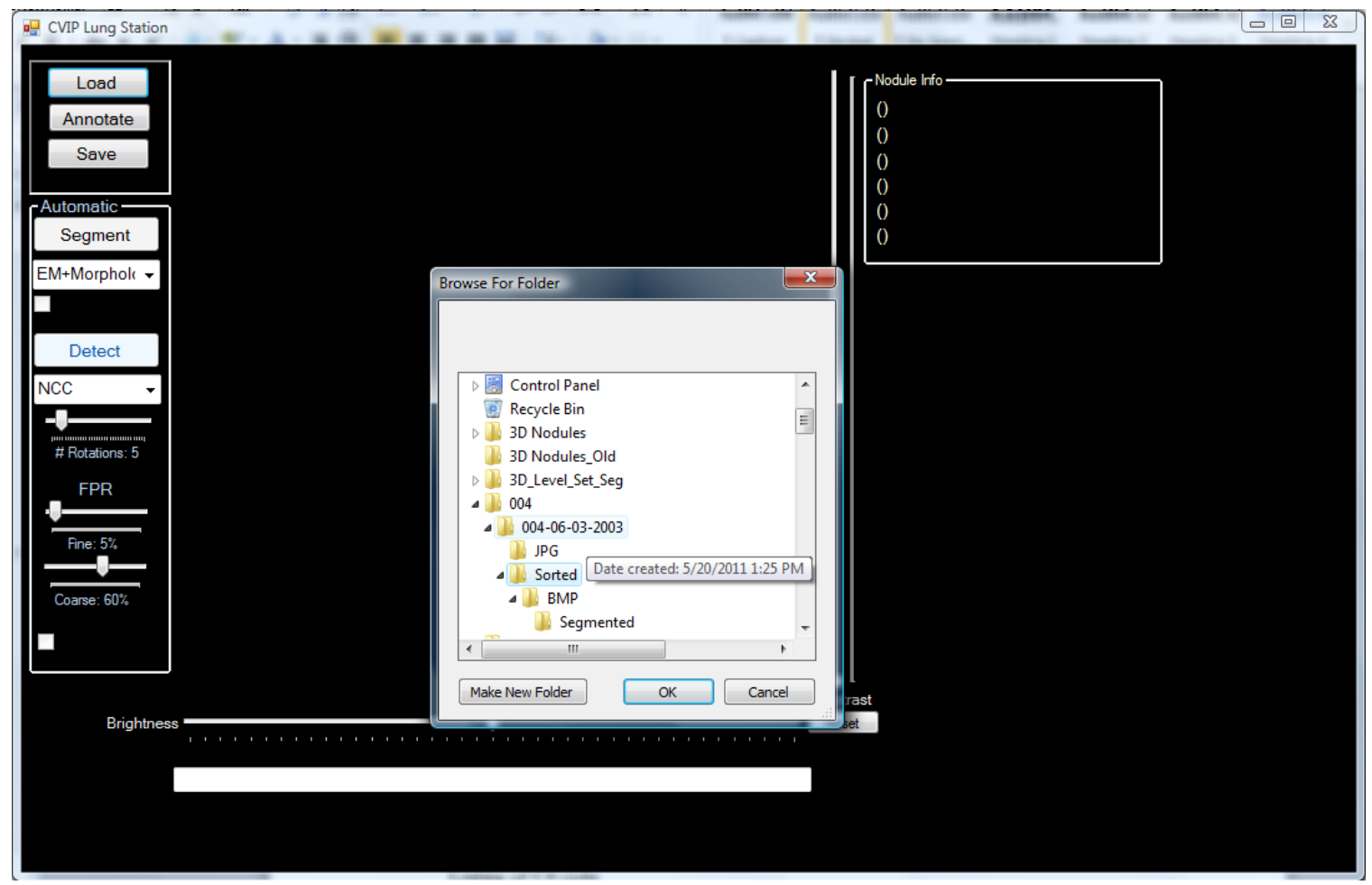

Figure 38: Screenshot of the interface prompting the user to load a CT scan for reading. This procedure is called by the Load button in the top, left-hand corner of the interface.

2.) Display the scan slice by slice in the Slice View panel, shown in Figure 39. Scrolling through slices has been designed for input from the mouse-wheel. Scrolling the mouse-wheel upwards/backwards displays slices of the scan respectively. Further, the Brightness and Contrast controls have been linked to the Slice View panel. Thus at any time during reading, the user may adjust or reset the image brightness and contrast using the horizontal and vertical sliders located below and to the right of the Slice View panel, respectively. This can also be seen in Figure 39. Once set, the brightness and contrast settings will be applied to each subsequent slice. This allows the physician to render the image to his/her liking and proceed with reading without having to constantly re-adjust the current slice. The brightness and contrast controls in 
the interface have been manually implemented as histogram thresholding and windowing. An example an adjusted image is displayed in Figure 40.

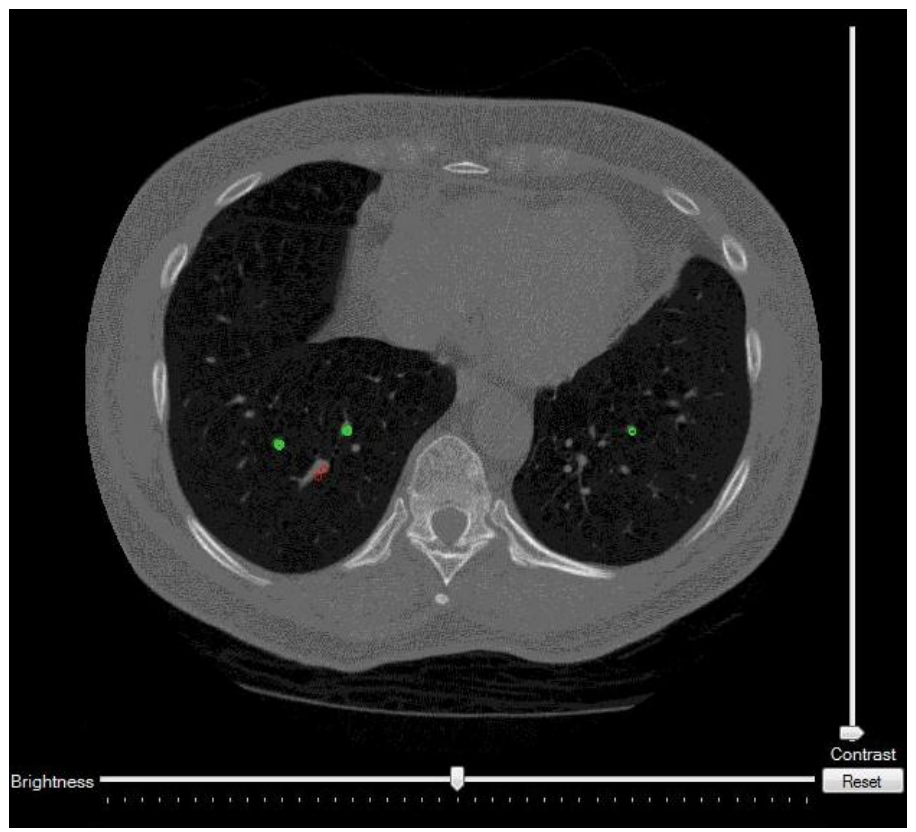

Figure 39: Screenshot of the Slice View panel. This panel occupies most of the interfaces working area. This design has been adopted to mimic currently used medical image reading software such as OsiriX, [ref OsiriX].

3.) If the user detects a nodule during reading, allow for nodule annotation using the Annotate button. When pressed, the Annotate button is highlighted in red, warning the user that they are currently annotating a nodule. This is shown in Figure 42. While in annotation mode, the first click in the Slice View panel will be stored as the $\mathrm{x}, \mathrm{y}$ location of the manually detected nodule. A red point is displayed in the Slice View panel to indicate the exact nodule location as clicked by the user. Furthermore, the current slice number is automatically stored and a cropped region around the manually detected nodule is displayed in the Crop View panel on the right side of the interface as shown in Figure 42. 
The user is then prompted to annotate the features described in Table $\mathbf{2}$ in a serial fashion using pop-up menus, like the one shown in Figure 41. Once the annotation is complete, a Nodule Info panel (top right panel of the interface) is filled with text outlining the characteristics annotated by user. This is shown throughout the later figures in this chapter.

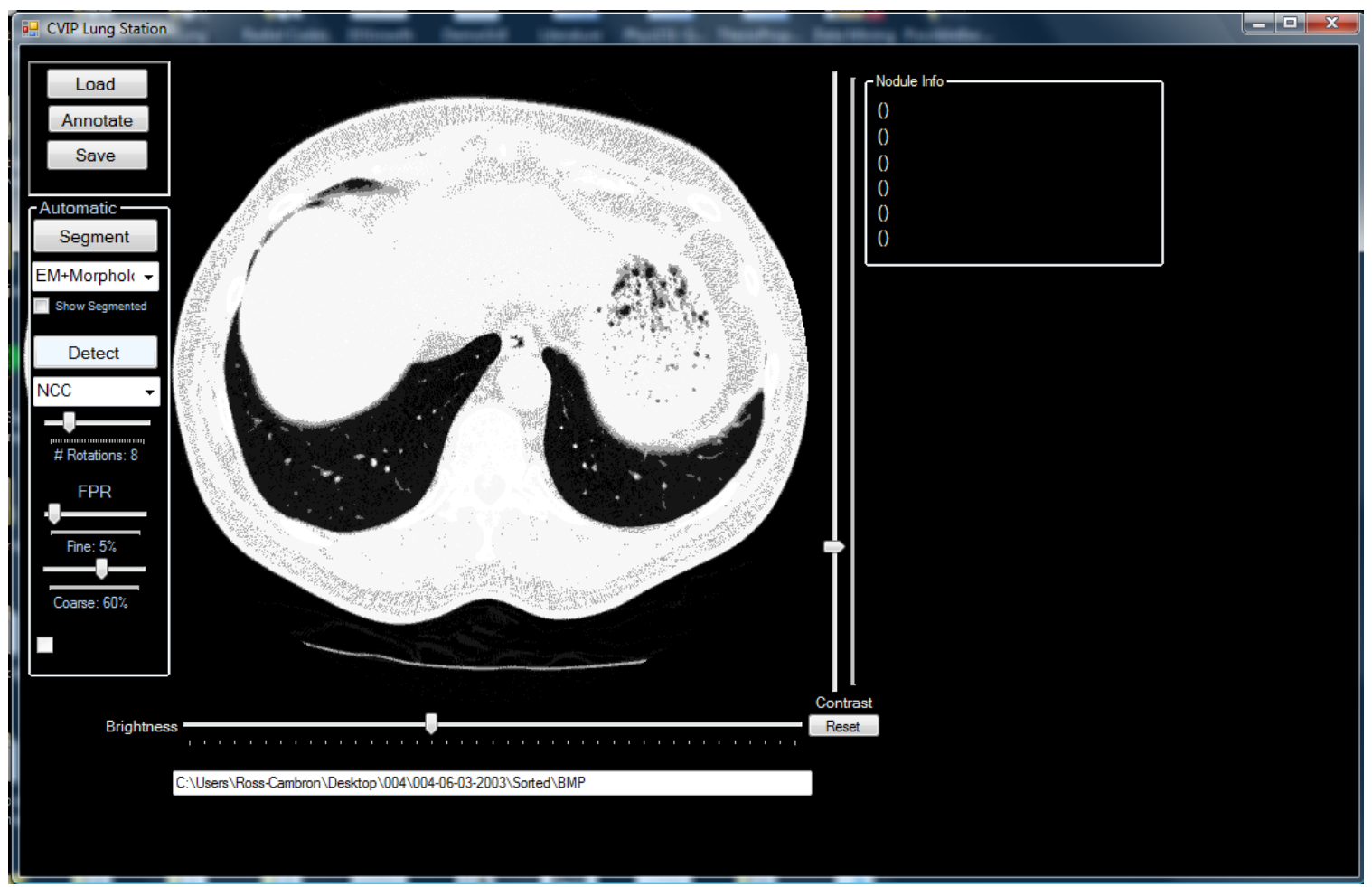

Figure 40: An image that has been adjusted using the Brightness and Contrast sliders located below and to the right of the interface. It should be noted that, once toggled, these settings are applied to each subsequent scan during reading. This allows the physician to adjust the image to his/her liking once and then proceed with reading, undisturbed.

4.) As the user continues to read through the scan, any previously annotated nodule will be displayed with a red point like the one shown in Figure 39. This is to remind the user if a nodule has already been annotated on the current slice. Further, if the user right-clicks the red point, a cropped region around 
the previously annotated nodule is displayed in the Crop View panel and the previously annotated nodule characteristics are displayed in the Nodule Info panel. This is shown in the following figures throughout this chapter.

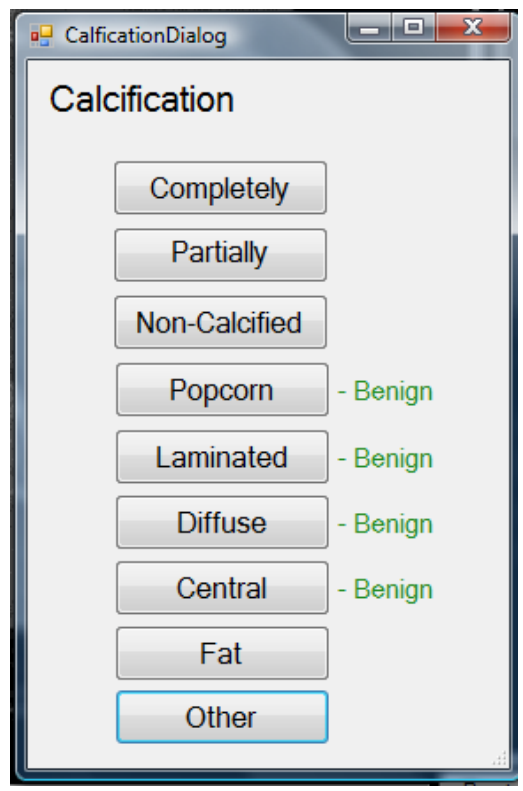

Figure 41: Pop-up menu which prompts the user to designate which Calcification pattern a manually detected nodule belongs to. Pop-up menus are used to grab all features outlined in Table 2 and are retrieved in a serial fashion-forcing the user to tag the nodule according to the profile described in Chapter 1.

All of these features outlined in Chapter $\mathbf{1}$ are stored in a structure designed for this system, called Nodule. This structure holds all relevant information regarding the manually annotated nodule. Once the scan is read in its entirety, all manually annotated nodules are saved as Nodule structures. Using the Save button, the user can store manual annotation data for the current scan. The annotations are stored as a List of Nodule structures and serialized to XML format for later retrieval. A sample XML file is given in Appendix A. 


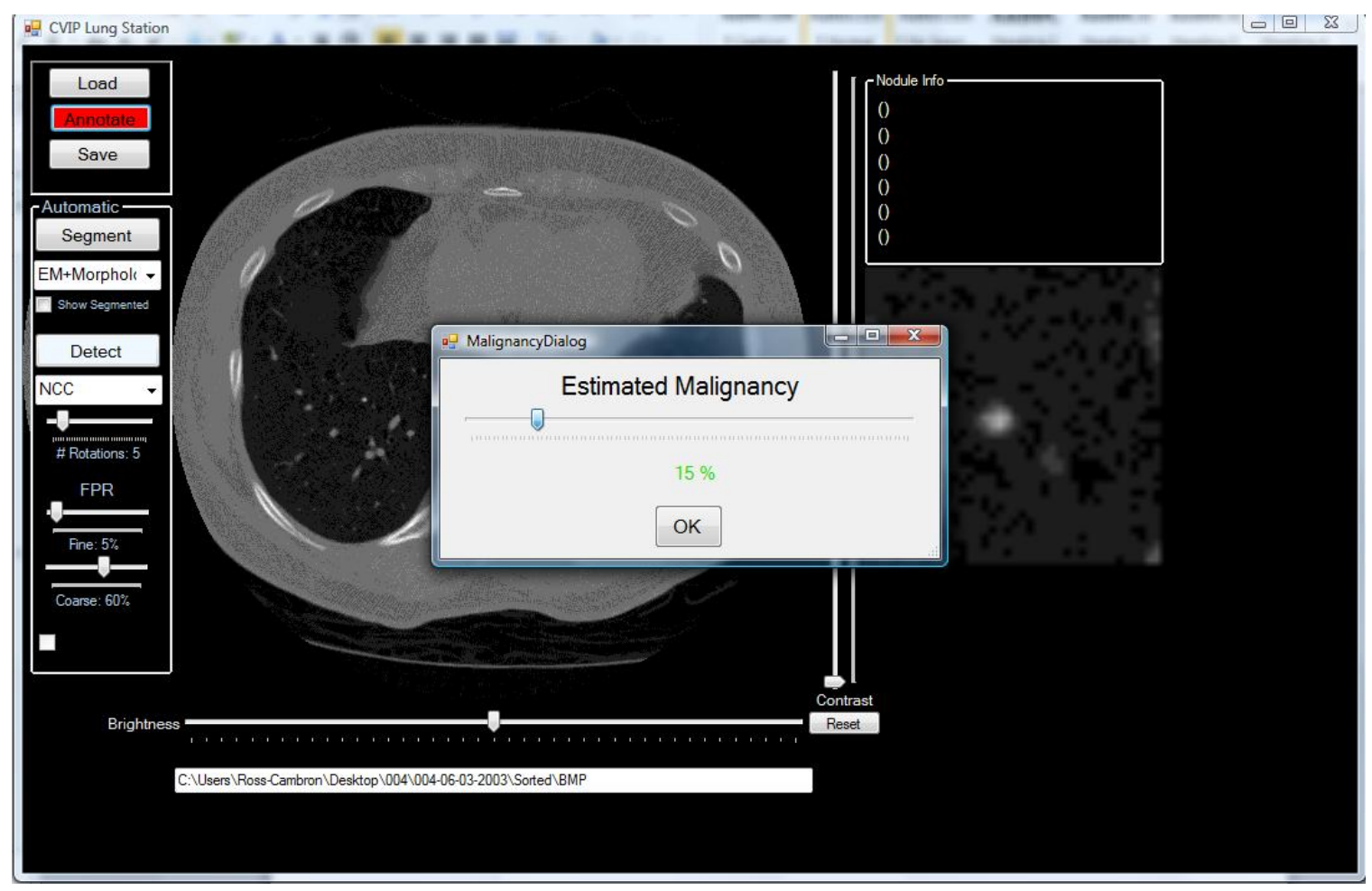

Figure 42: Screenshot of a user annotating the Estimated Malignancy of a manually detected nodule. Notice the Annotate button in the top right-hand toolbox is highlighted in red, indicating to the user that the interface is currently in annotation mode. Also notice the cropped region around the nodule is automatically displayed in the Crop View panel on the right-hand side of the interface.

\subsubsection{Enrolling a Nodule in Modeling Dataset}

When a nodule is detected in the CAD system, whether it be manually or automatically (described below), the option to enroll such nodule into a modeling dataset is then presented to the user. The modeling dataset is a subset of annotated nodules which may be later used to generate new models for detection as discussed in Chapter 3. Both the AAM and ASM approaches used in [Amal ref] require further control point annotation of members that are to be considered in the modeling process. The Model button appears once a nodule has been annotated by the user or selected by the user with a right-click. This is shown in Figure 37. The goal of this feature is to allow the user to 
generate their own nodule templates (models) which can then be used for automatic detection in the reading of later scans. The default templates used by the detection process have been generated using the ELCAP database in [ref Amal modeling].

If the user chooses to enroll the currently selected nodule into a modeling dataset, they may simply click the Model button, which triggers the appearance of a second window. This window prompts the user to identify which of the four Kostis categories the current nodule should be treated as. This is shown in Figure 44. Once a category is selected, the user is prompted to annotate ten control points needed for AAM and ASM. The control points for each category are shown below in Figure 43.

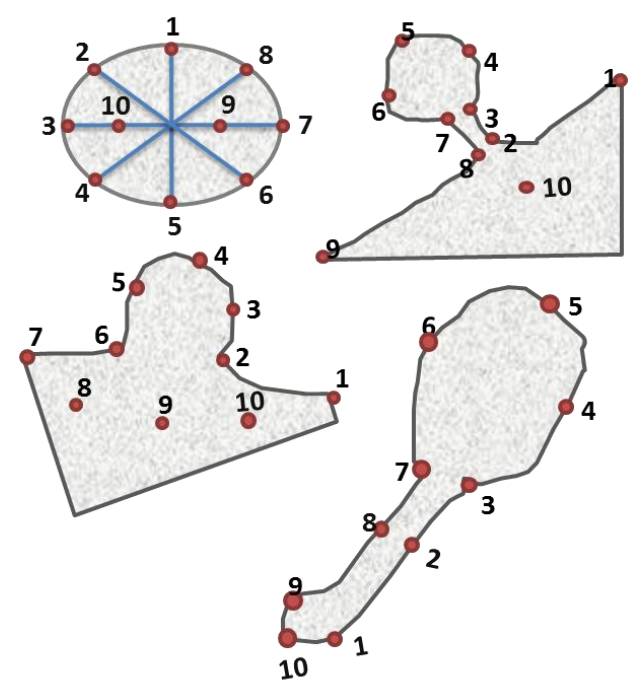

Figure 43: Templates showing control points needed for AAM and ASM which need to be manually annotated by an expert. The control points are shown for Well (top-left), Tail (top-right), Juxta (bottom-left) and Vascularized (bottom-left).

It should be noted that for this thesis, the nodule models only pertain to these four categories, however the framework could be extended to handle the modeling of various nodule characteristics. 


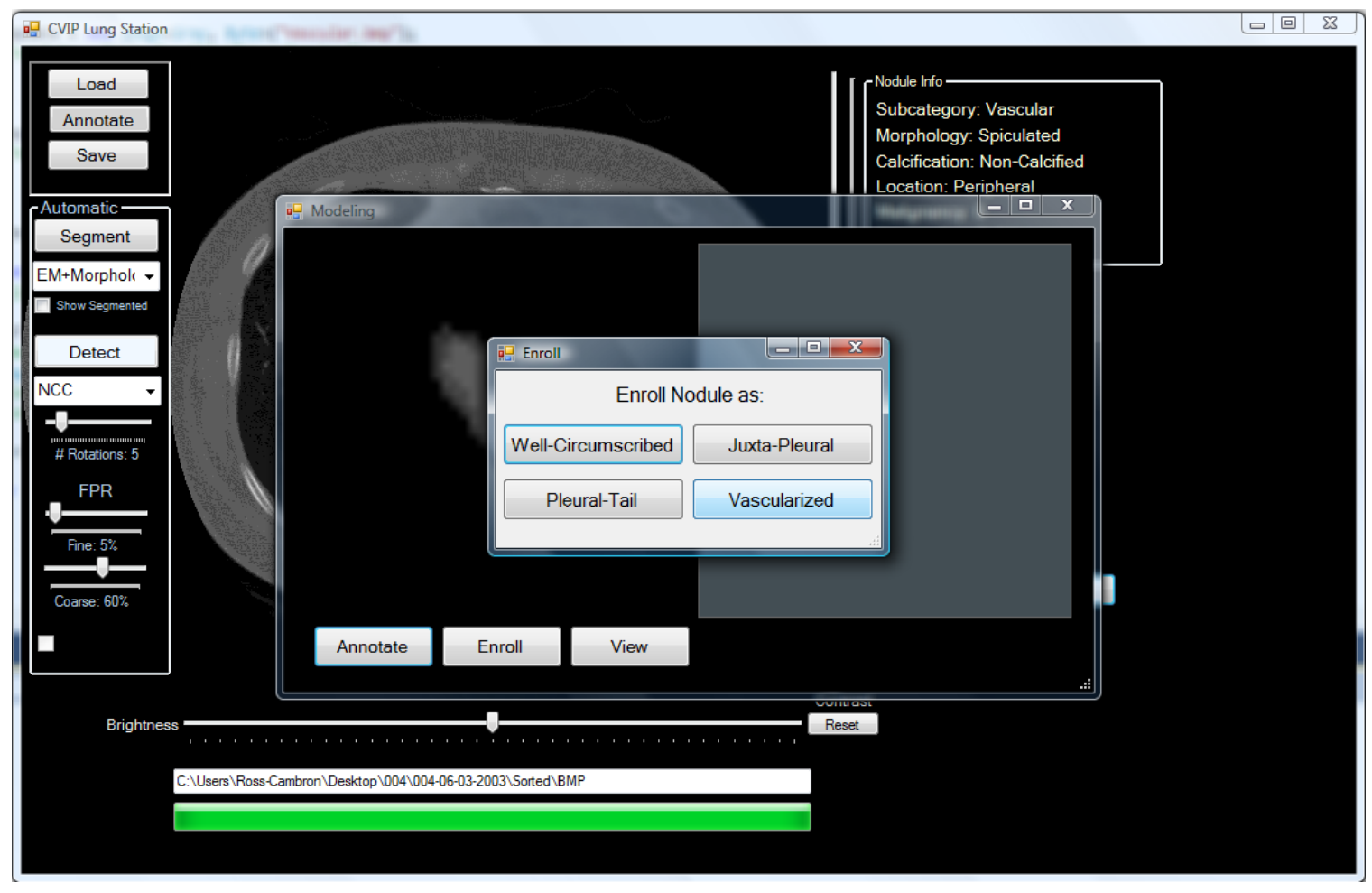

Figure 44: Modeling window that is triggered by clicking the Model button. This button appears once a nodule has been selected by the user and allows for the enrollment of the current nodule into a sub-database of nodules that will be later used for generating new models.

Selecting the appropriate nodule category displays a cropped region around the current nodule in a viewing panel with an example control-point diagram displayed directly to its right. This diagram is meant to guide the user through control-point annotation. The Annotate button in this new window beings the process of collecting the ten controlpoints from the user via left-clicking. Once annotation is complete, the user confirms enrollment into the modeling dataset using the Enroll button. This is all shown in Figure 45. 


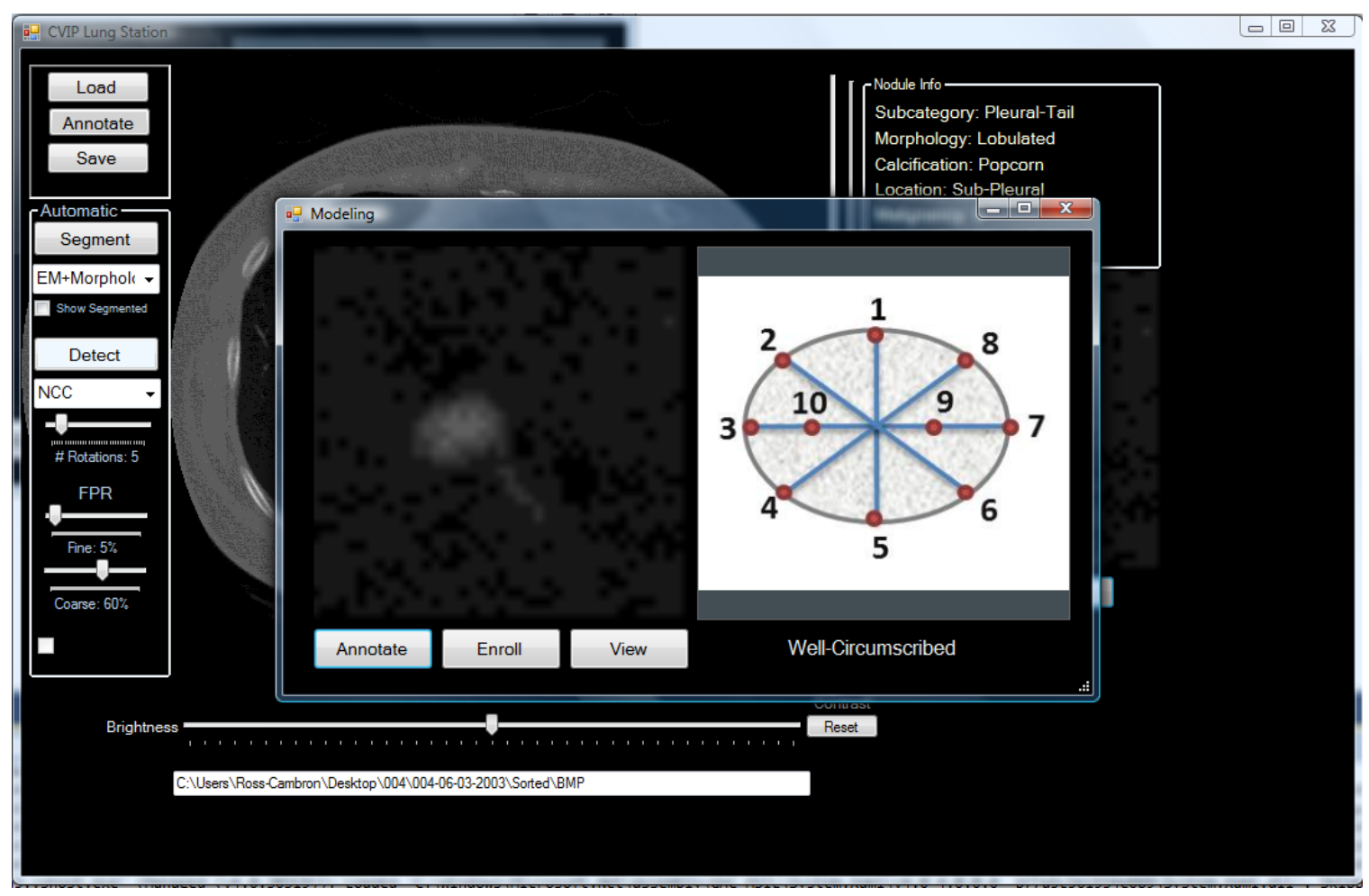

Figure 45: Window used for obtaining manually annotated control-points from the user to feed the AAM and ASM process in later stages of modeling. The current nodule is displayed in the left viewing panel and an example diagram of control points for the appropriate nodule category is displayed in the right viewing panel.

\subsection{Tissue Segmentation}

Segmenting the lung tissue is a very straightforward process in this interface.

Once a scan is loaded, as in Section 6.2, the user has the option to segment out the tissue from the raw CT using the Segment button, located in the Automatic Toolbox, located on the left-side of the interface. A drop down menu has been placed underneath the Segment button to allow the user choice of segmentation method. The choices are listed as described in Chapter 2 but, as a result of the modular design of this system, adding new segmentation methodologies to the interface in the future is extremely feasible. 
Clicking the Segment button triggers the interface to begin segmenting the entire scan. The segmentation progress is displayed in a progress bar located at the bottom of the interface, below the Slice View panel. The most important feature of the tissue segmentation implemented in this interface is that it is carried out as a background process, using a separate thread from the main interface. This is made possible with the use of a feature called BackgroundWorker in Visual C\# [ref BackgroundWorker]. Thus, the user can continue reading the scan while tissue segmentation occurs in the background. Further, tissue segmentation results are stored upon completion. In this way, the user only has to segment a scan once unless the tissue segmentation results are undesirable. For the EM-based segmentation process, slices are segmented in parallel as segmentation results from one slice do not affect the next. This greatly enhances the speed of EM-based tissue segmentation in the interface. Sample runtimes for three different scans are shown below in Table 8.

\begin{tabular}{|l|l|l|l|}
\hline Method & Scan 1 (474 slices) & Scan 2 (174 slices) & Scan 3 (113 slices) \\
\hline EM Algorithm & $5.01 \mathrm{~min}$ & $1.94 \mathrm{~min}$ & $1.56 \mathrm{~min}$ \\
\hline 3-D Level Sets & $25.44 \mathrm{~min}$ & $10.97 \mathrm{~min}$ & $6.96 \mathrm{~min}$ \\
\hline
\end{tabular}

Table 8: Tissue Segmentation runtimes for 3 individual scans with 474, 174, and 113 slices respectively. Runtimes are tabulated for the segmentation of the entire scan.

Once tissue segmentation has been carried out, a check box option becomes available in the Automatic Toolbox labeled Show Segmented. Enabling this checkbox display only the isolated lung tissue in the Slice View panel. This is shown in Figure 46 and Figure 47. 


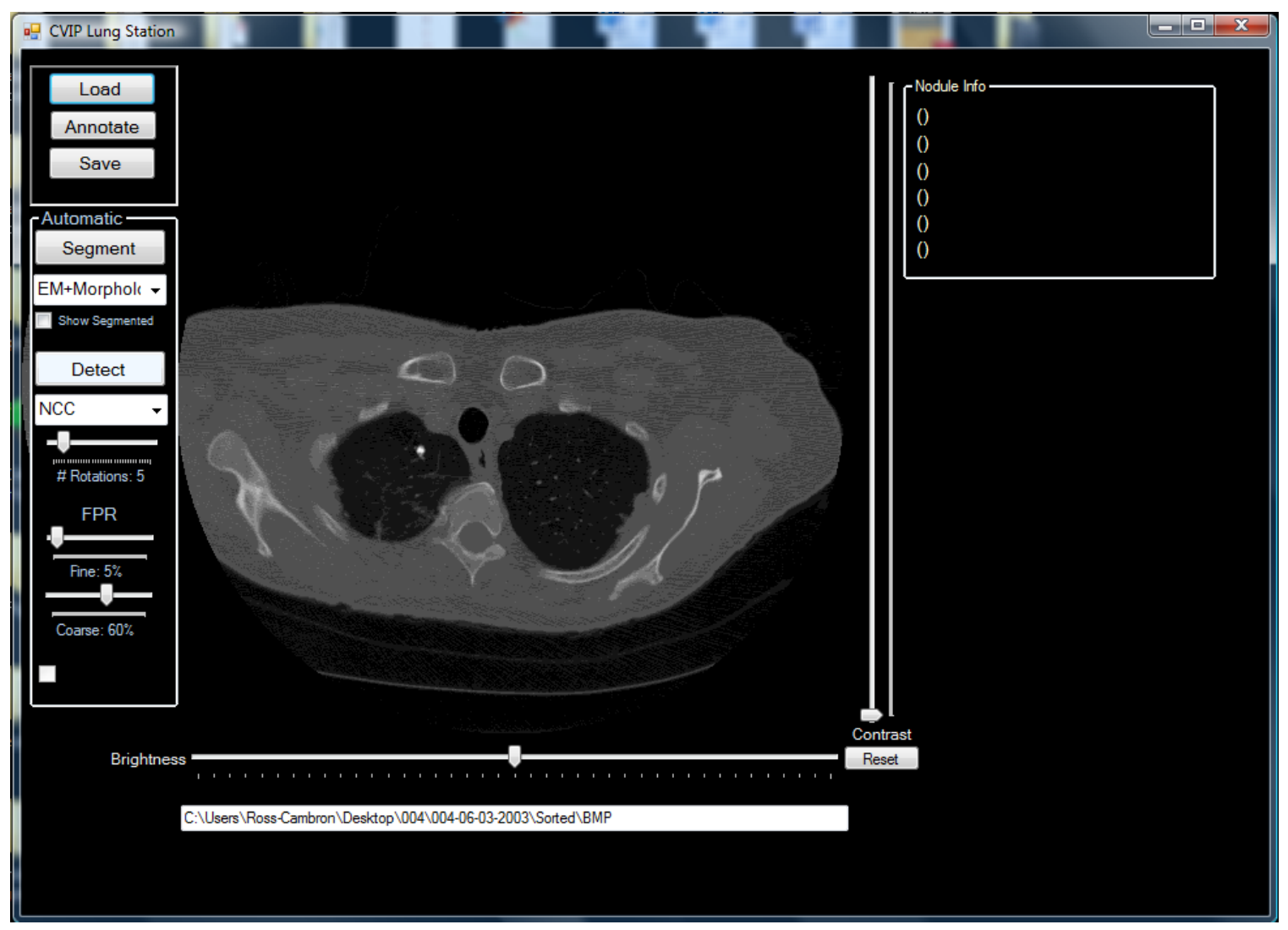

Figure 46: Viewing a raw $\mathrm{CT}$ slice in a scan that has previously undergone tissue segmentation. Notice the Show Segmented check box in the Automatic Toolbox. Enabling this check box isolates the lung tissue in the Slice View Panel.

It should be noted that the brightness and contrast features are still available when viewing the isolated lung tissue. As the user toggles the mouse-wheel, only lung tissue is displayed provided that the Show Segmented check box is enabled. 


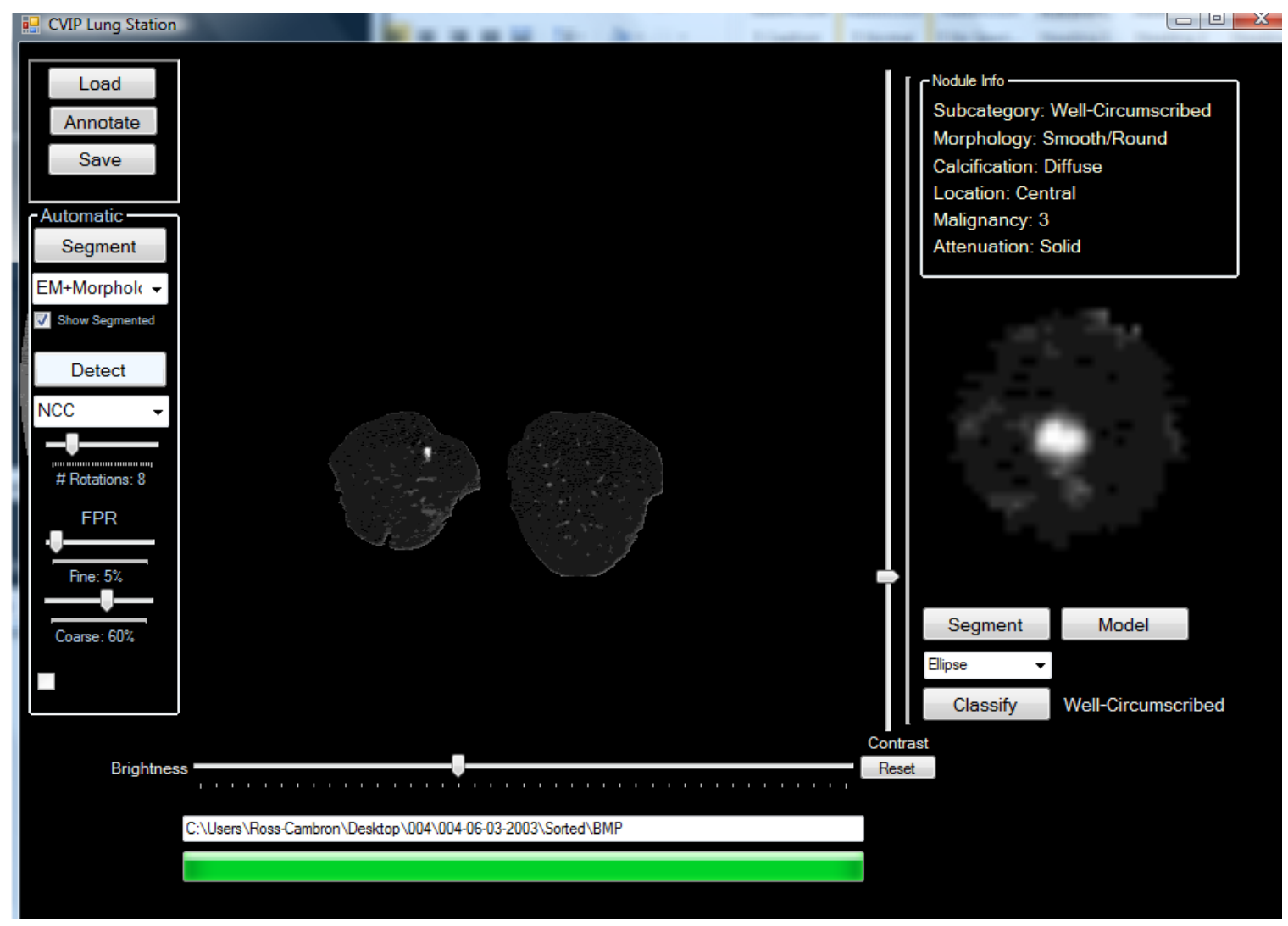

Figure 47: The same slice from Figure 46 with the Show Segmented check box enabled. This feature only displays the lung tissue from the current slice in the Slice View panel.

\subsection{Nodule Detection}

The automatic nodule detection scheme in outlined in Chapter $\mathbf{3}$ is made available to the user in the Automatic Toolbox. The toolbox offers options to toggle coarse and fine false positive reduction as discussed in Chapter $\mathbf{3}$ and tune the number of rotations considered for the template matching procedure. A drop down menu is provided in the toolbox which allows the user to select the distance measure to be used in the automatic detection process. The toolbox is shown in Figure 48. 


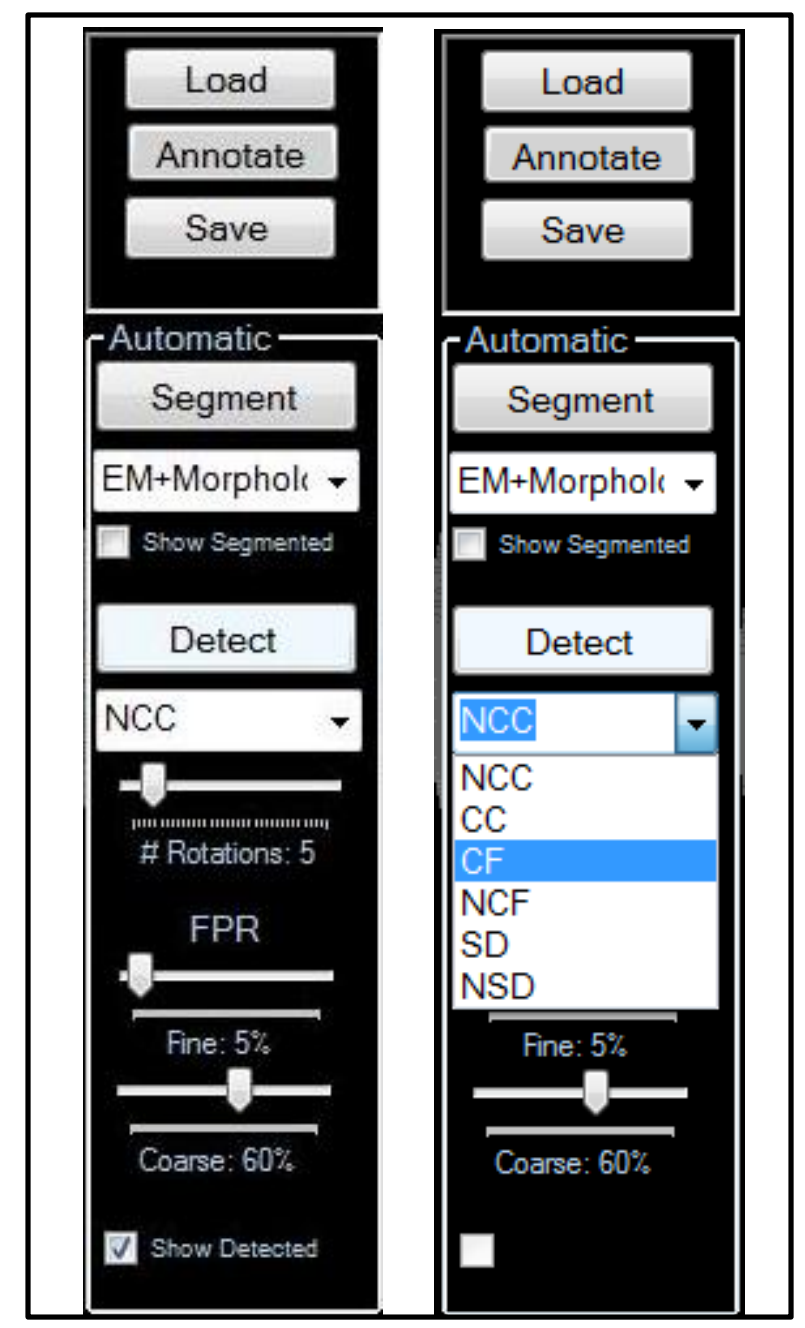

Figure 48: (Left) Detailed view of the Automatic Toolbox on the left-side of the interface. (Right) Illustration of the drop down menu which allows the user to select the distance measure used in template matching during automatic detection.

The most notable detection feature in the Automatic Toolbox is the ability to adjust the sensitivity of false positive reduction. Figure 21 outlines this procedure. Toggling both the coarse and fine sensitivity (discussed in Chapter 3) is done using two sliders, located under the labeled region of the toolbox, FPR. An illustration of the interfaces handling of false positives via these sliders is illustrated in the figures below. Detection is first carried out on a single slice using the NCC, with a Coarse FPR threshold of 0.11 and a Fine FPR percentage set to $67 \%$. This all correlations at or below 0.11 are truncated and 
omitted and further, only the remaining top $67 \%$ of the candidate nodules are retained.

The initial detection is shown in Figure 49. Detection is triggered with the Detect button located in the Automatic Toolbox and the resulting, detected nodules are displayed as green points in the Slice View panel overtop the current slice.

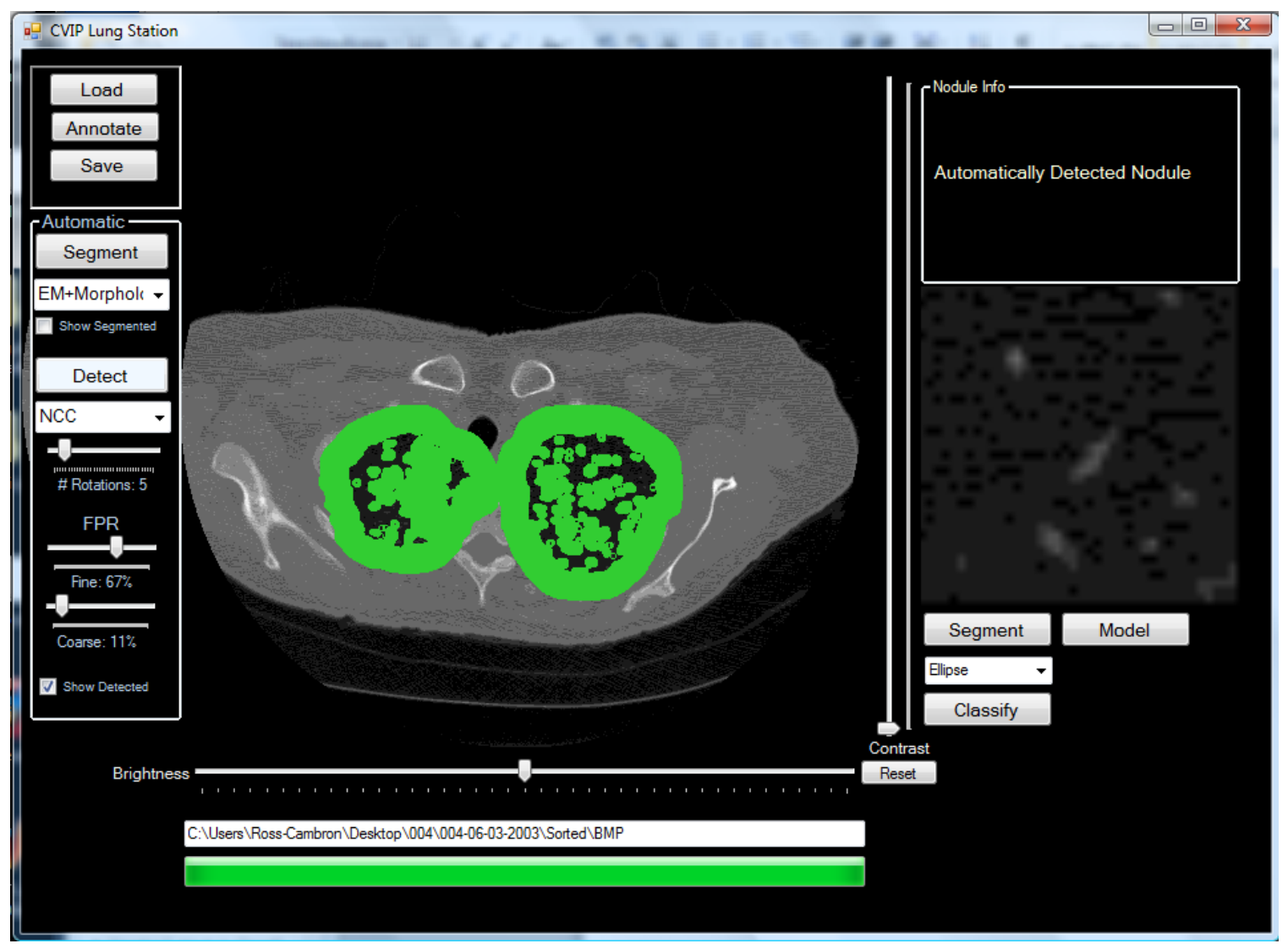

Figure 49: Nodule detection carried out using the NCC with a Coarse FPR sensitivity set to 0.11 and a Fine FPR sensitivity set to $67 \%$. The results are riddled with false positives. Green points in the Slice View panel represent detected nodules.

Upon inspection of the initial detection results, these FPR settings are not stringent enough to provide any assistance to the operating physician as there are far too many false positives. Using the available FPR adjustments in the CAD system, these false positives can be greatly reduced. Figure 51 illustrates the functionality of the Fine FPR 
sensitivity. Here, the NCC is used, the Coarse FPR sensitivity is set to 0.12 and the Fine FPR sensitivity is reduced to $5 \%$.

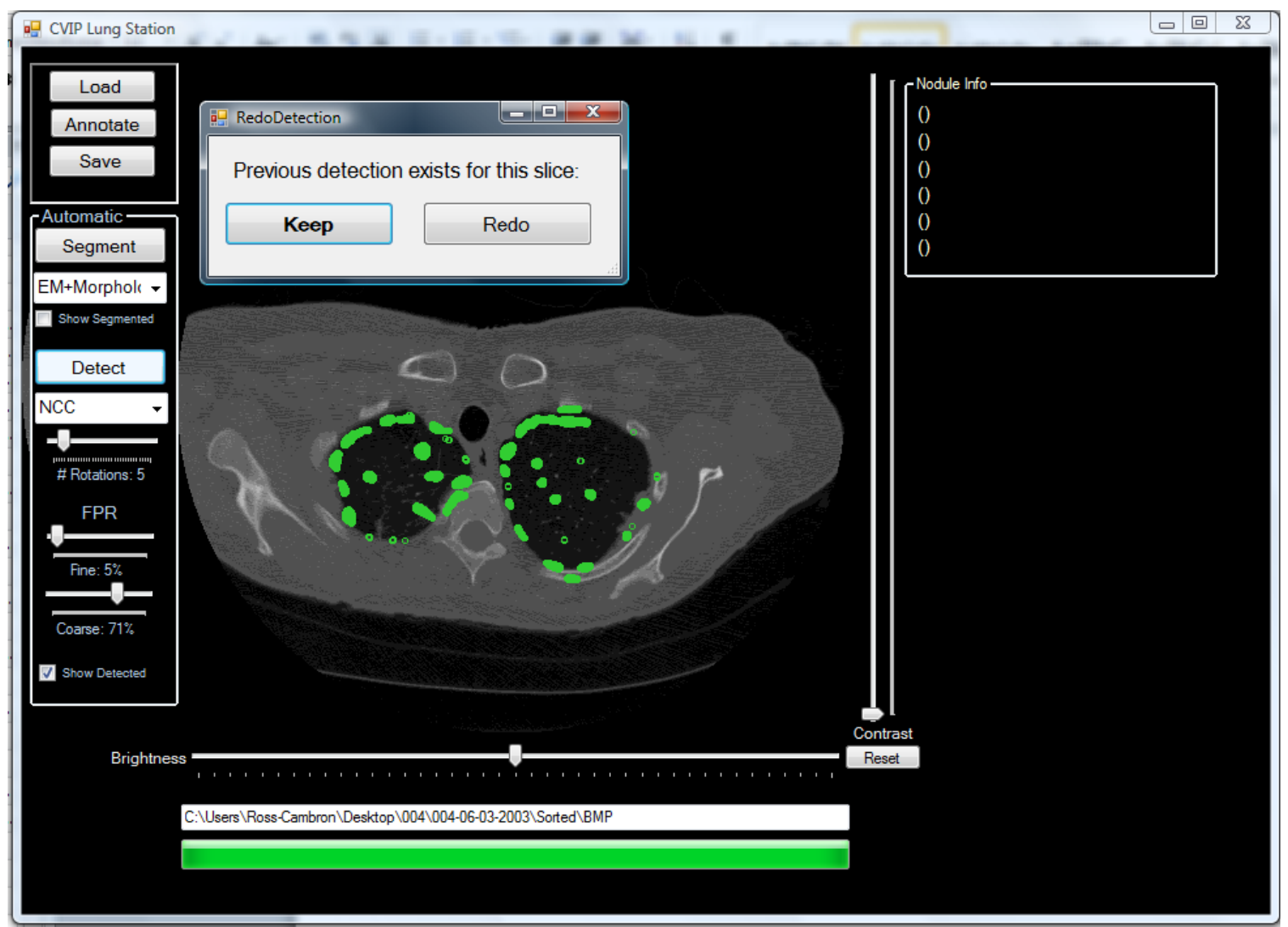

Figure 50: The interface seeks confirmation from the user if they intend on repeatedly carrying out detection on the same slice.

An additional feature is displayed in Figure $\mathbf{5 0}$ which seeks user confirmation to overwrite detection results in a slice that has already undergone automatic detection. Detection results are stored in a similar fashion as with the manual annotation data and loaded with scans that have already had detection carried out on them. Thus, a user can be warned before they overwrite their previous annotation results. 


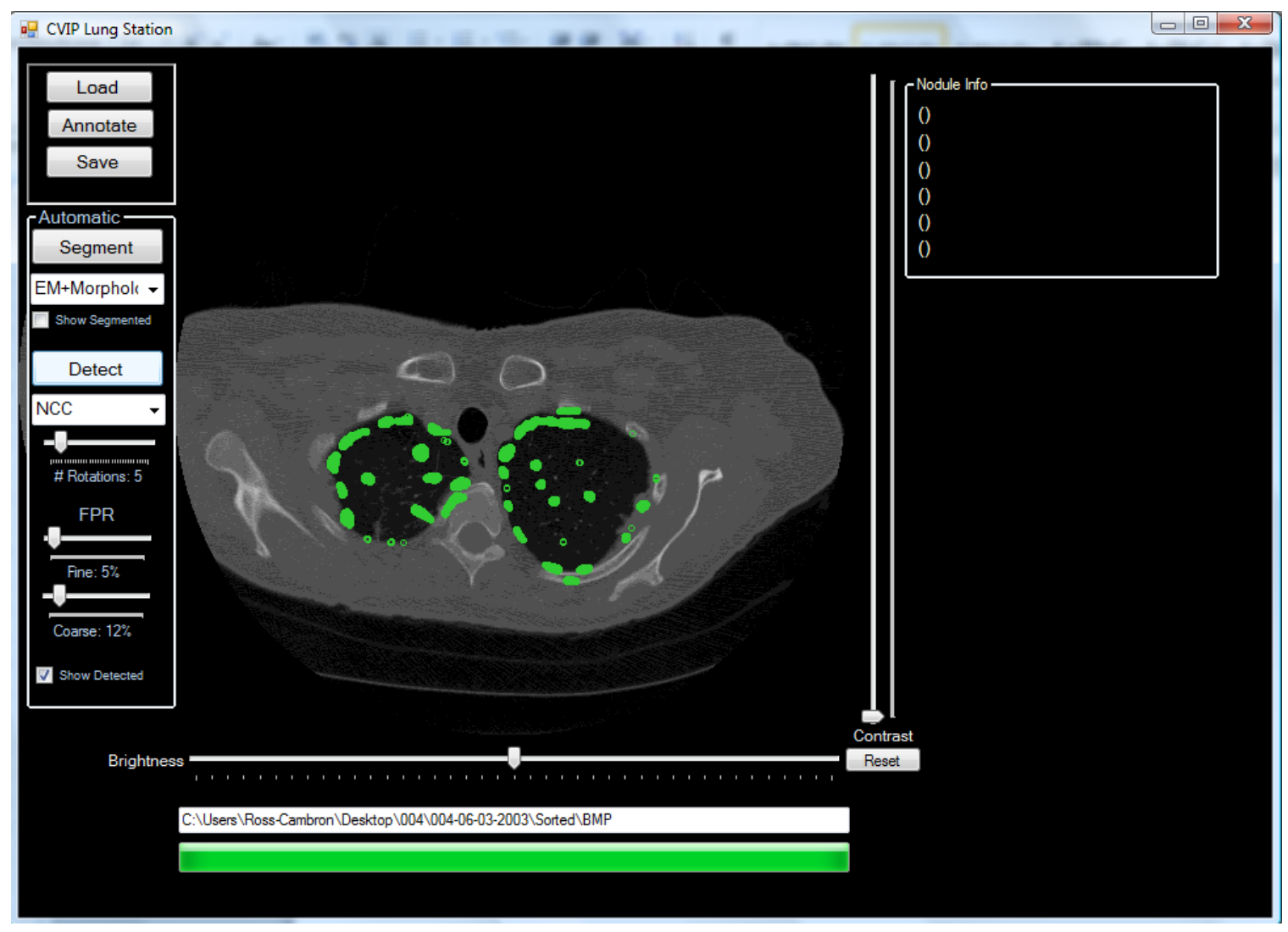

Figure 51: Updated detection results for the same slice operated on in Figure 49. The NCC is still used, however the Fine FPR sensitivity has been reduced to only display the top $5 \%$ of detected nodules. The resulting, detected nodules are shown in green.

This reduction greatly enhances the detection results by omitting false positives. The results in Figure 51 are far more desirable than those in Figure 49, however the number of false positives is likely still too high to provide a physician with any real assistance. To further remove false positives the Coarse FPR sensitivity can be employed. Setting the Coarse FPR sensitivity to 0.6 removes all false positives and leaves only detected points around a true nodule. The Well-Circumscribed nodule exists in the right lung and is detected by the system as shown in Figure 52. 


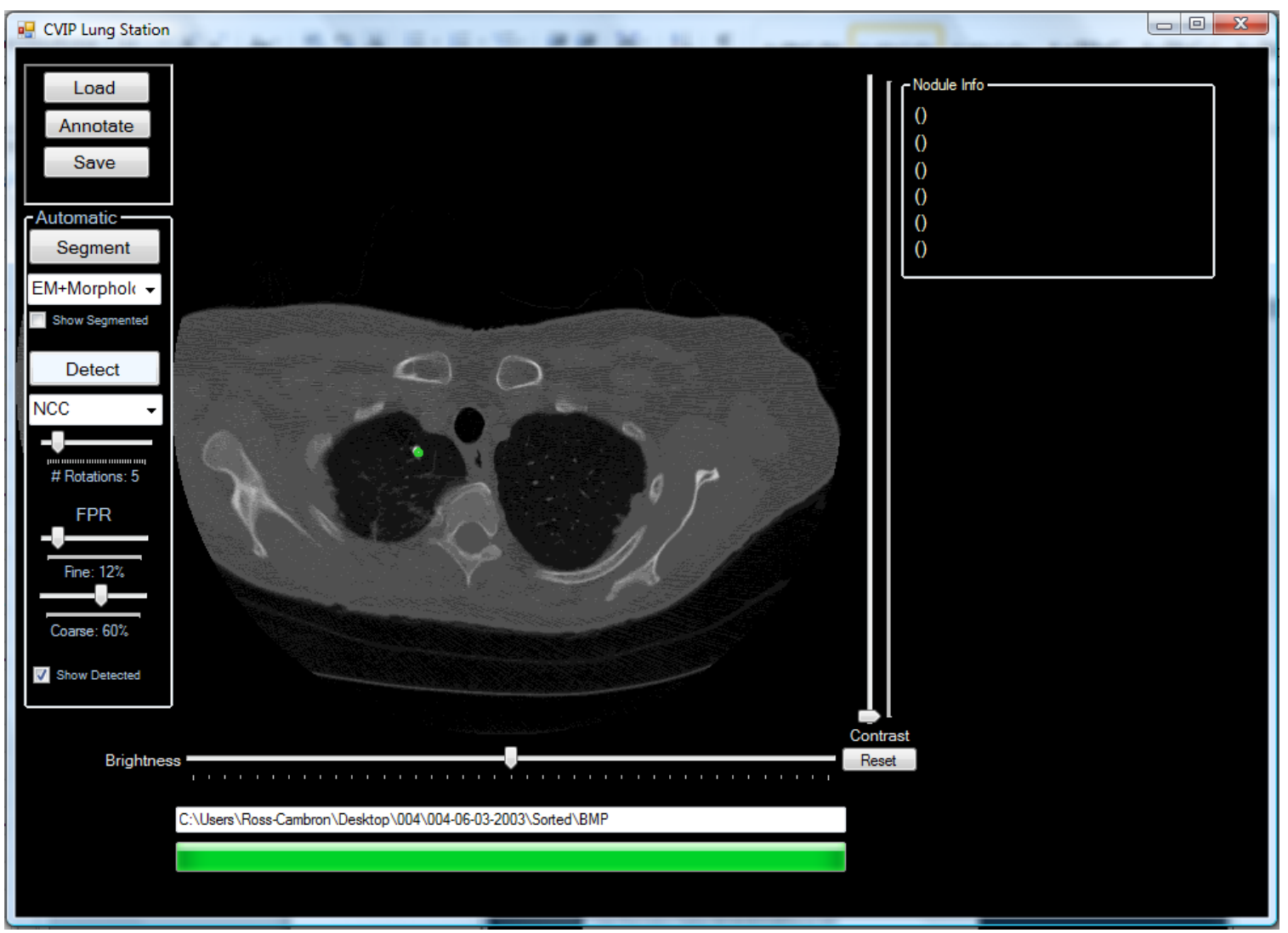

Figure 52: Updated detection results using the NCC with a Coarse FPR sensitivity of 0.6 and a Fine FPR sensitivity of $12 \%$. The detection results yield a cluster of detected points surrounding a true, Calcified, Well-Circumscribed nodule. No false positives remain.

More detection results are shown in Appendix A. Table 9 gives a listing of detection runtimes for all distance measures with varying numbers of rotations considered.

\begin{tabular}{|l|l|l|l|l|l|l|}
\hline \# Rot. $\left(^{\circ}\right)$ & NCC & CC & CF & NCF & SD & NSD \\
\hline $\mathbf{5}$ & $4.296 \mathrm{~s}$ & $4.516 \mathrm{~s}$ & $3.724 \mathrm{~s}$ & $3.575 \mathrm{~s}$ & $6.906 \mathrm{~s}$ & $4.481 \mathrm{~s}$ \\
\hline $\mathbf{1 0}$ & $6.267 \mathrm{~s}$ & $8.227 \mathrm{~s}$ & $7.318 \mathrm{~s}$ & $6.777 \mathrm{~s}$ & $10.34 \mathrm{~s}$ & $8.741 \mathrm{~s}$ \\
\hline $\mathbf{1 5}$ & $9.341 \mathrm{~s}$ & $12.949 \mathrm{~s}$ & $11.028 \mathrm{~s}$ & $9.963 \mathrm{~s}$ & $15.071 \mathrm{~s}$ & $13.027 \mathrm{~s}$ \\
\hline $\mathbf{2 0}$ & $12.327 \mathrm{~s}$ & $16.793 \mathrm{~s}$ & $14.958 \mathrm{~s}$ & $13.361 \mathrm{~s}$ & $21.295 \mathrm{~s}$ & $17.932 \mathrm{~s}$ \\
\hline $\mathbf{2 5}$ & $15.499 \mathrm{~s}$ & $20.482 \mathrm{~s}$ & $18.515 \mathrm{~s}$ & $16.829 \mathrm{~s}$ & $25.915 \mathrm{~s}$ & $21.601 \mathrm{~s}$ \\
\hline
\end{tabular}




\begin{tabular}{|l|l|l|l|l|l|l|}
\hline 30 & $18.419 \mathrm{~s}$ & $24.954 \mathrm{~s}$ & $21.497 \mathrm{~s}$ & $19.545 \mathrm{~s}$ & $31.513 \mathrm{~s}$ & $26.05 \mathrm{~s}$ \\
\hline 35 & $21.316 \mathrm{~s}$ & $29.67 \mathrm{~s}$ & $26.051 \mathrm{~s}$ & $31.016 \mathrm{~s}$ & $48.609 \mathrm{~s}$ & $37.99 \mathrm{~s}$ \\
\hline
\end{tabular}

Table 9: Average runtimes per slice for nodule detection using each distance measure in the CAD system. The runtimes were averaged from individual runtimes taken from 20 slices at random from the same scan.

\subsection{Nodule Segmentation and Classification}

The nodule segmentation and classification tools appear on the right-side of the interface once a user has selected an annotated/detected nodule. The two buttons Segment and Classify allow the user to call these procedures. A drop down menu is available for the selection of nodule segmentation methods. The method choices are those listed in Chapter 4. Nodule segmentation is carried out on the cropped region displayed in the Crop View panel and subsequent results are also displayed here. Classification results are displayed as text next to the Classify button. This is all shown in Figure 53. 
Nodule Info

Subcategory: Well-Circumscribed

Morphology: Smooth/Round

Calcification: Non-Calcified

Location: Central

Malignancy: 0

Attenuation: Solid
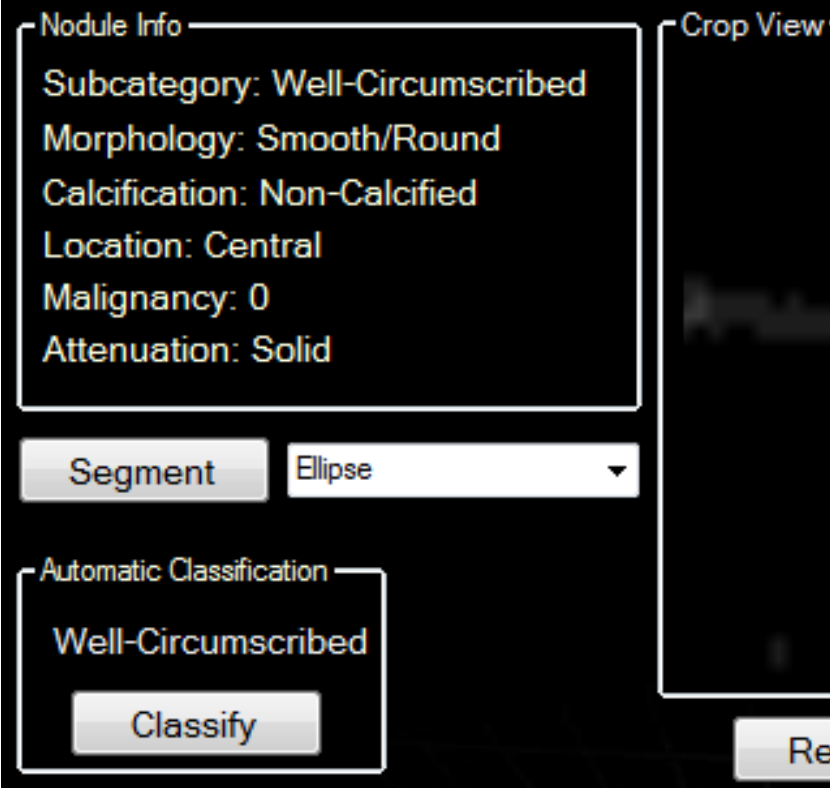

Remove

Model

Figure 53: Right-hand side of the interface which handles the segmentation and classification of a nodule after it has been detected or annotated. The segmentation results are directly displayed in the Crop View panel and classification results are shown as text next to the Classify button.

Table 10 shows nodule segmentation runtimes for ten different nodules. Runtimes are displayed in seconds.

\begin{tabular}{|l|c|c|c|c|c|c|c|c|c|c|}
\hline Nodule & $\mathbf{1}$ & $\mathbf{2}$ & $\mathbf{3}$ & $\mathbf{4}$ & $\mathbf{5}$ & $\mathbf{6}$ & $\mathbf{7}$ & $\mathbf{8}$ & $\mathbf{9}$ & $\mathbf{1 0}$ \\
\hline Ellipse & 1.146 & 1.073 & 3.063 & 1.06 & 1.057 & 4.964 & 1.049 & 1.091 & 2.482 & 1.971 \\
\hline EM & 0.062 & 0.006 & 0.006 & .007 & 0.061 & 0.006 & 0.006 & 0.006 & 0.01 & 0.007 \\
\hline $\begin{array}{l}\text { Ellipse } \\
+ \text { EM }\end{array}$ & 1.103 & 1.088 & 3.074 & 1.054 & 1.07 & 4.931 & 1.055 & 1.11 & 2.497 & 1.999 \\
\hline $\begin{array}{c}\text { Level } \\
\text { Sets }\end{array}$ & 0.086 & 0.052 & 0.122 & 0.055 & 0.034 & 0.038 & 0.085 & 0.052 & 0.051 & 0.046 \\
\hline
\end{tabular}

Table 10: Nodule Segmentation runtimes in seconds for 10 different candidate nodules automatically detected by the CAD system. Candidate nodules were taken from the same scan.

Runtimes are displayed in seconds. 
Table 11 shows nodule classification runtimes for ten different nodules. Runtimes are displayed in milliseconds.

\begin{tabular}{|l|c|c|c|c|c|c|c|c|c|c|}
\hline Nodule & $\mathbf{1}$ & $\mathbf{2}$ & $\mathbf{3}$ & $\mathbf{4}$ & $\mathbf{5}$ & $\mathbf{6}$ & $\mathbf{7}$ & $\mathbf{8}$ & $\mathbf{9}$ & $\mathbf{1 0}$ \\
\hline $\begin{array}{l}\text { Time } \\
(\mathbf{m s})\end{array}$ & 60 & 49 & 50 & 49 & 49 & 50 & 53 & 50 & 49 & 48 \\
\hline
\end{tabular}

Table 11: Classification runtimes in milliseconds for 10 different candidate nodules automatically detected by the CAD system. Candidate nodules were taken from the same scan and runtimes are displayed in milliseconds. 


\section{Conclusion}

The components needed to design a functional CAD system using CT for lung nodule analysis have been presented. Multiple solutions have been provided for each stage of the pipeline: Tissue Segmentation, Nodule Detection and Nodule Classification. The system has been designed to handle these solutions in a modular fashion, allowing the operating physician the freedom to select the method of choice for each task. The goal of this thesis was to consolidate the tools needed for each of these stages in the pipeline into a functional and usable user interface. This CAD system has been designed and outlined in detail in Chapter 6. Each component of the CAD system is functional and provides the user with options and safeguards. The system has been designed to handle scans that have already been read using the system, giving the physician the option to review prior annotations, as well as new scans. Runtimes for each component have been tabulated and shown to be clinically viable.

In short, the system prototyped in this thesis lays the foundation for a lung nodule analysis tool which provides physicians with a second-opinion detection and diagnosis scheme using state-of-the-art Computer Vision, Pattern Recognition, and programming techniques. The system is functional, modular and fast. 


\section{References}

1. The United States Department of Health and Human Services (NIH); www.nih.gov

2. Swensen, S. J. MD, James R. Jett, MD, Thomas E. Hartman, MD, David E. Midthun, MD, Jeff A. Sloan, PhD, Anne-Marie Sykes, MD, Gregory L. Aughenbaugh, MD and Medy A. Clemens, CCRP, Lung Cancer Screening with CT: Mayo Clinic Experience March 2003 Radiology, 226, 756-761.

3. The National Cancer Institute; www.cancer.gov

4. W. J. Kostis, et al., "Small pulmonary nodules: reproducibility of three-dimensional volumetric measurement and estimation of time to follow-up," Radiology, Vol. 231, pp. 446-52, 2004.

5. Amal Farag, Lung Nodule Modeling and Detection for Computerized Image Analysis of Low Dose CT Imaging of the Chest. Master of Engineering, University of Louisville, May 2009.

6. ELCAP public lung image database. www.via.cornell.edu/databases/lungdb.html

7. American Cancer Society; www.cancer.org

8. Farag, A. A., Modeling Small Object Under Uncertainties: Novel Algorithms and Applications. PhD Dissertation, May 2012

9. Helen Hong, Jeongjin Lee, Yeny Yim, Automatic lung nodule matching on sequential CT images, Computers in Biology and Medicine, Volume 38, Issue 5, May 2008, Pages 623-634, ISSN 0010-4825, 10.1016/j.compbiomed.2008.02.010.

10. G. Armato, G. McLennan, M. F. McNitt-Gray, C. R. Meyer, D. Yankelevitz, D. R. Aberle, C. I. Henschke, E. A. Hoffman, E. A. Kazerooni, H. MacMahon, A. P. Reeves, B. Y. Croft, and L. P. Clarke, "Lung image database consortium: developing a resource for the medical imaging research community," Radiology, vol. 232, no. 3, pp. 739-748, 2004. (LIDC)

11. Hardie RC, Rogers SK, Wilson T, Rogers A., Performance analysis of a new computer aided detection system for identifying lung nodules on chest radiographs. Medical Image Anaysis 2008 June; 12(3):240-58. Epub 2007 Oct 25. 
12. Dolejsi, M. Kybic, J. Tuma, S. Polovincak, M, Reducing false positive responses in lung nodule detector system by asymmetric adaboost. 5th IEEE International Symposium on Biomedical Imaging: From Nano to Macro, 2008. ISBI 2008.

13. Hosseini, R, Salah D. Qanadli, Sarah Barman, Mahdi Mazinani, Tim Ellis, Jamshid Dehmeshki, An Automatic Approach for Learning and Tuning Gaussian Interval Type-2 Fuzzy Membership Functions Applied to Lung CAD Classification System, IEEE Transactions on Fuzzy Systems, April 2012, 224-234

14. T. Cootes, G. Edwards and C. Taylor, "Active Appearance Models," Proc. European Conference on Computer Vision, ECCV’98, pp. 484-498, 1998.

15. T. Cootes, "An introduction to active shape models," - Chapter 7 in Model-Based Methods in Analysis of Biomedical Images, Ed. R. Baldock and J. Graham, Oxford University Press, 2000, pp223-248.

16. I. Mathews and S. Baker, Active Appearance Models Revisited, International Journal of Computer Vision, Vol. 60, No. 2, November, 2004, pp. 135 - 164.

17. M.A.O. Vasilescu, D. Terzopoulos, "Multilinear Projection for Appearance-Based Recognition in the Tensor Framework," Proc. Eleventh IEEE International Conf. on Computer Vision (ICCV'07), Rio de Janeiro, Brazil, October, 2007, 1-8.

18. Amal Farag, Ham Rara, Cambron Carter, Ahmed El-Barkouky, and Aly Farag, Experiments with Active Shape, Appearance and Tensor Modeling, for Faces and Objects - TR-April 2012, CVIP Lab, University of Louisville, April 2012.

19. Y. Lee, T. Hara, H. Fujita, S. Itoh and T. Ishigaki, "Automated detection of pulmonary nodules in helical CT images based on an improved template matching technique," IEEE Transactions on Medical Imaging, Vol. 20 pp. 595-604, 2001.

20. Amal Farag, James Graham, Aly Farag, Salwa Elshazly and Robert Falk. Parametric and Non-Parametric Nodule Models: Design and Evaluation, Proc. of Third International Workshop on Pulmonary Image Processing in conjunction with MICCAI-'10, Beijing, September 2010.

21. Leung, A., Smithuis, R. Solitary pulmonary nodule: benign versus malignant. Department of Radiology, Stanford University Medical Center, Stanford, California and the Department of Radiology, Rijnland Hospital, Leiderdorp, the Netherlands 
22. Xujiong Ye, Xinyu Lin, Jamshid Dehmeshki, Greg Slabaugh, and Gareth Beddoe, "Shape Based Computer-Aided Detection of Lung Nodules in Thoracic CT Images", IEEE Transactions on Biomedical Engineering, 56(7), pp: 1810-20, 2009.

23. McCulloch, C.C., Kaucic, R.A., Mendonça, P.R.S., Walter, D.J., Avila, R.S.: Modelbased detection of lung nodules in computed tomography exams. Academic Radiology 11(3) (2004) 258-266

24. I. Sluimer, A. Schilham, M. Prokop, and B. van Ginneken, "Computer Analysis of Computed Tomography Scans of the Lung: A Survey," IEEE Transactions on Medical Imaging, vol. 25, No. 4, pp. 385-405, April,2006.

25. D. G. Lowe, "Distinctive Image Features from Scale-Invariant Keypoints", International Journal of Computer Vision, 60, 2, pp. 91-110, 2004.

26. J.M. Morel and G. Yu., "ASIFT: A New Framework for Fully Affine Invariant Image Comparison," SIAM Journal on Imaging Sciences, pp. 438-469, 2009.

27. K. Murphy, B. van Ginneken, A.M.R. Schilham, B.J. de Hoop, H.A. Gietema, M. Prokop, "A large-scale evaluation of automatic pulmonary nodule detection in chest CT using local image features and k-nearest-neighbour classification", Medical Image Analysis, Volume 13, Issue 5, October 2009, Pages 757-770.

28. The United States Department of Health and Human Services (NIH); www.nih.gov

29. Amal A. Farag, S.Y. Elhabian, S.A. Elshazly and A.A. Farag, "Quantification of Nodule Detection in Chest CT: A Clinical Investigation Based on the ELCAP Study," Proc. of International Workshop on Pulmonary Image Processing in conjunction with MICCAI-09, Sept. 2009, pp. 149-160.

30. Samala, R., et al., "A Novel Approach to Nodule Feature Optimization on Thin Section Thoracic CT.” Acad. Radiology. Vol. 15, pp.1181-1197.2009

31. N. Paragios, R. Deriche, "Active Contours and Level Sets for the Detection and Tracking of Moving Objects”, PAMI, No. 3, March 2000, pp. 266-280.

32. H. Abdelmunim and A. A. Farag, "Shape Representation and Registration using Vector Distance Functions," Proc. of IEEE Conference on Computer Vision and Pattern Recognition (CVPR'07), Minneapolis, MN, June 18-23, 2007.

33. A. Farag, A. El-Baz, G. L. Gimel'farb, "Precise segmentation of multimodal images," IEEE Transactions on Image Processing Vol. 15, no. 4, April 2006, pp. 952-968.

34. A.P. Reeves, A.B. Chan, D.F. Yankelevitz, C.I. Henschke, B. Kressler, and W.J. Kostis, "On measuring the change in size of pulmonary nodules." IEEE Transactions on Medical Imaging, 25(4):435-450, 2006. 
35. MacMahon H, Austin JH, Gamsu G, et al. "Guidelines for management of small pulmonary nodules detected on CT scans: a statement from the Fleischner Society," Radiology 2005; 237:395-400.

36. Jane P. Ko, Henry Rusinek, Erika L. Jacobs, James S. Babb, Margrit Betke, Georgeann McGuinness, and David P. Naidich, "Small Pulmonary Nodules: Volume Measurement at Chest CT-Phantom Study," Radiology, pp: 864-870, 2003.

37. J.M. Kuhnigk, V. Dicken, L. Bornemann, A. Bakai, D. Wormanns, S. Krass, and H.O. Peitgen, "Morphological segmentation and partial volume analysis for volumetry of solid pulmonary lesions in thoracic CT scans."IEEE Transactions on Medical Imaging, 25(4):417434, 2006.

38. Armato, S. G. 3rd, Giger, M. L., Moran C. J., Blackburn, J. T., Doi, K., MacMahon $\mathrm{H}$, "Computerized detection of pulmonary nodules on CT scans," Journal of Radio Graphics, Vol. 19 pp.1303-1311 (1999).

39. Stefano Diciotti, Giulia Picozzi, Massimo Falchini, Mario Mascalchi, Natale Villari, and Guido Valli, "3-D Segmentation Algorithm of Small Lung Nodules in Spiral CT Images," IEEE Transactions on Information Technology in Biomedicine, 12(1):7-19, 2008.

40. Artit C. Jirapatnakul, Yury D. Mulman, Anthony P. Reeves, David F. Yankelevitz, and Claudia I. Henschke, "Segmentation of Juxtapleural Pulmonary Nodules Using a Robust Surface Estimate." International Journal of Biomedical Imaging, Volume 2011, Article ID 632195, 2011.

41. R. Redner and H. Walker, Mixture densities, maximum likelihood and the EM algorithm (review), SIAM Rev., vol. 26, pp. 195-237, 1984.

42. Amal Farag, James Graham, and Aly Farag, "Robust Segmentation of Lung Tissue In Chest CT Scanning," Proc. of 2010 IEEE International Conference on Image Processing (ICIP), pp. 2249-2252, 2010.

43. Amal Farag, James Graham and Aly Farag, "Statistical Modeling of the Lung Nodules in Low Dose Computed Tomography Scans of the Chest," Proc. of 2010 IEEE International Conference on Image Processing (ICIP), pp. 4281-4284, 2010.

44. Asem Ali, Amal Farag and Aly Farag, "Labelling Color Images by Modelling the Colors Density Using A Linear Combination of Gaussians and EM Algorithm" 2009 IEEE International Conference on Image Processing (ICIP), Nov. 7 - Nov. 10, 2009, Cairo, Egypt.

45. Hossam Abd EL Munim and Aly A. Farag, "Curve/Surface Representation and Evolution using Vector Level Sets with Application to the Shape-based Segmentation Problem," IEEE Transactions on Pattern Analysis and Machine Intelligence - Vol. 29, No. 6, pp. 945-958, June 2007. 
46. T. Ojala, M. Pietikainen, and T. Maenpaa, "Multiresolution gray-scale and rotation invariant texture classification with local binary patterns" in IEEE Transactions on Pattern Analysis and Machine Intelligence, 24, 2002, pp. 971-987

47. Amal Farag, Asem Ali, Shireen Elhabian, James Graham, Aly Farag, and Robert Falk, "Feature-Based Lung Nodule Classification," Proc. of International Symposium on Visual Computing (ISVC), pp. 79-88, 2010.

48. Amal Farag, Shireen Elhabian, James Graham, Aly Farag, and Robert Falk, "Toward Precise Pulmonary Nodule Descriptors for Nodule Type Classification,” Proc. of the 13th International Conference on Medical Image Computing and Computer Assisted Intervention (MICCAI), pp. 626-633, 2010.

49. Amal Farag, Asem Ali, James Graham, Aly Farag, Salwa Elshazly and Robert Falk. "Feature-Based Nodule Classification and Detection in Low Dose CT scans of the human chest." ISBI 2011

50. Amal A. Farag, Aly A. Farag, Hossam Abdelmunim, Asem M. Ali, James Graham, Salwa Elshazly, Ahmed Farag, Sabry Al Mogy ,Mohamed Al Mogy, Sahar Al Jafary, Hani Mahdi, Robert Falk and Rebecca Milam. "Feature Descriptors for Nodule Type Classification”. CARS 2011.

51. MATLAB Skin Detection Tutorial:

http://se.cs.ait.ac.th/cvwiki/matlab:tutorial:detectface

52. OsiriX DICOM Viewer; www.osirix-viewer.com 


\section{Appendix A:}

A sample XML file written to store manually annotated nodules for a given scan:

$<$ ?xml version="1.0" encoding="utf-8"?>

<ArrayOfNodule xmlns:xsi="http://www.w3.org/2001/XMLSchema-instance" xmlns:xsd="http://www.w3.org/2001/XMLSchema">

$<$ Nodule>

$<$ Calcification $>$

$<$ string $>$ Partially Calcified $</$ string $>$

$</$ Calcification $>$

$<$ Attenuation>

$<$ string $>$ Mixed-Solid</string $>$

$</$ Attenuation $>$

$<$ Morphology >

<string $>$ Lobulated $</$ string $>$

$</$ Morphology $>$

<AnatomicalLocation>

$<$ string $>$ Sub-Pleural $</$ string $>$

</AnatomicalLocation>

$<$ Subcategory >

$<$ string $>$ Pleural-Tail $</$ string $>$

</Subcategory>

<Malignancy>

$<$ double $>0</$ double $>$

</Malignancy>

$<$ Location>

$<$ Point $>$ 


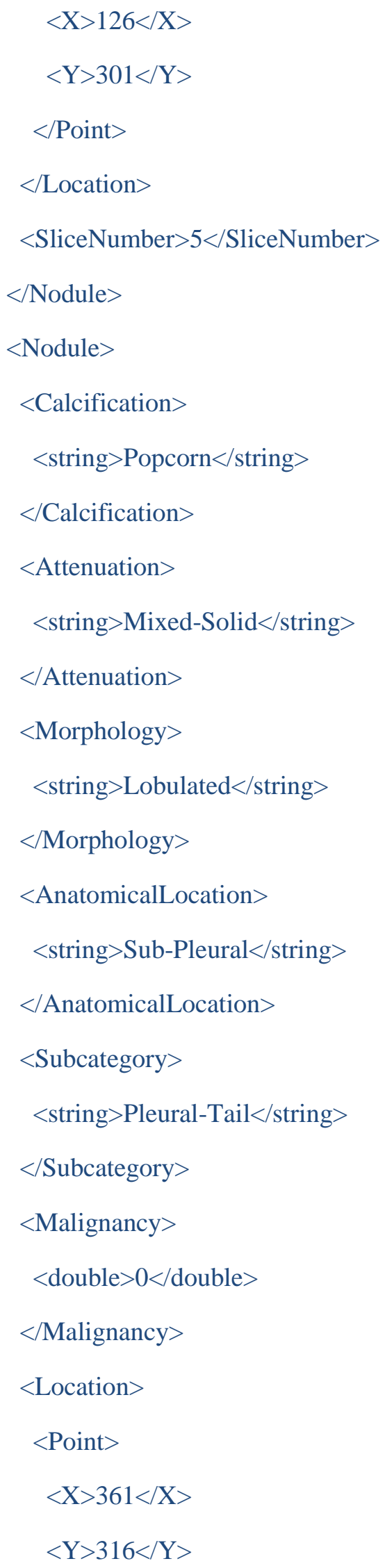


$</$ Point $>$

$</$ Location $>$

$<$ SliceNumber $>7</$ SliceNumber $>$

$<$ /Nodule >

</ArrayOfNodule> 


\section{A.1 Extra Figures}

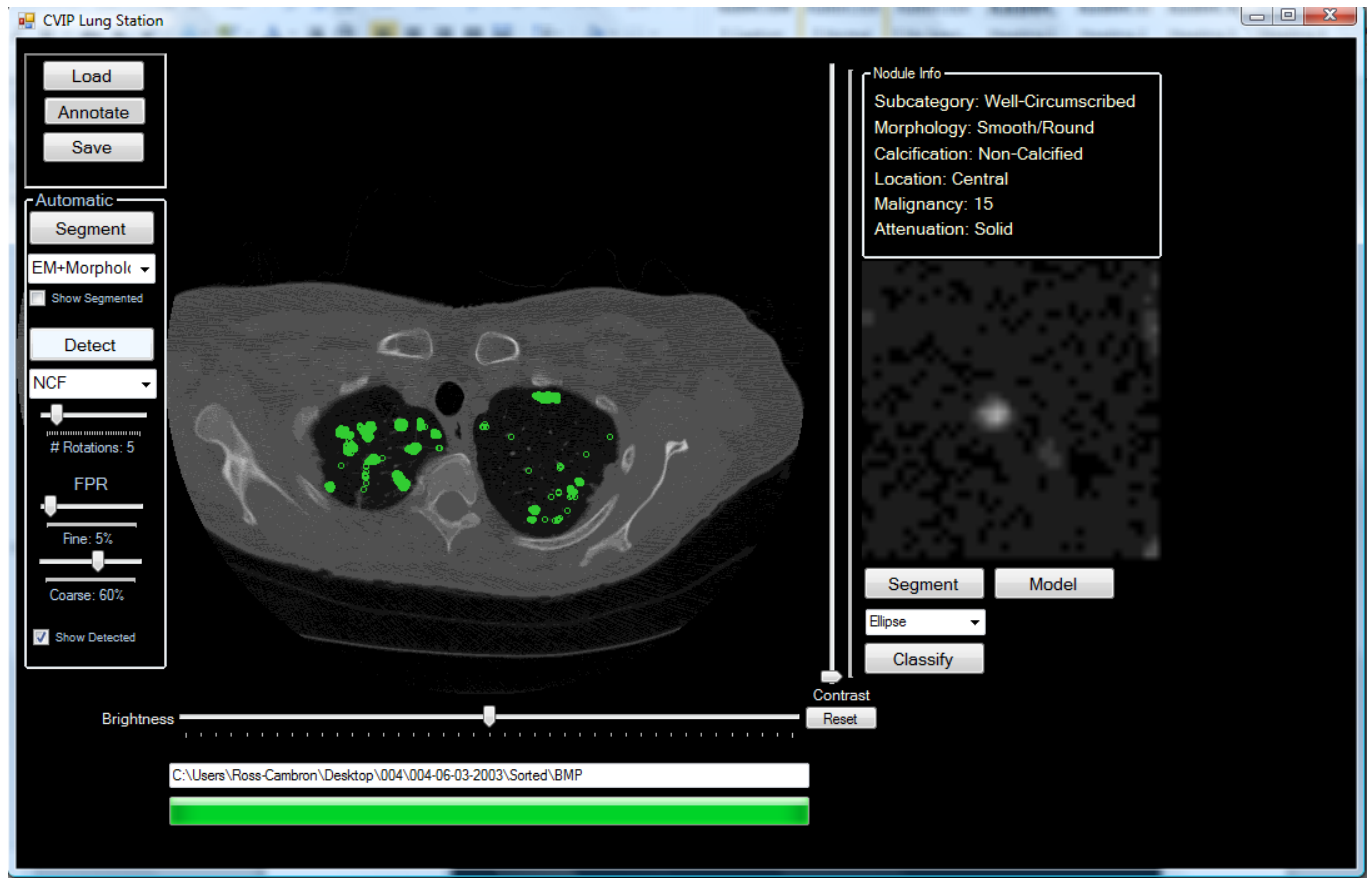

Figure 54: Nodule detection carried out using the NCF with a Coarse FPR sensitivity set to 0.6 and a Fine FPR sensitivity set to $5 \%$.

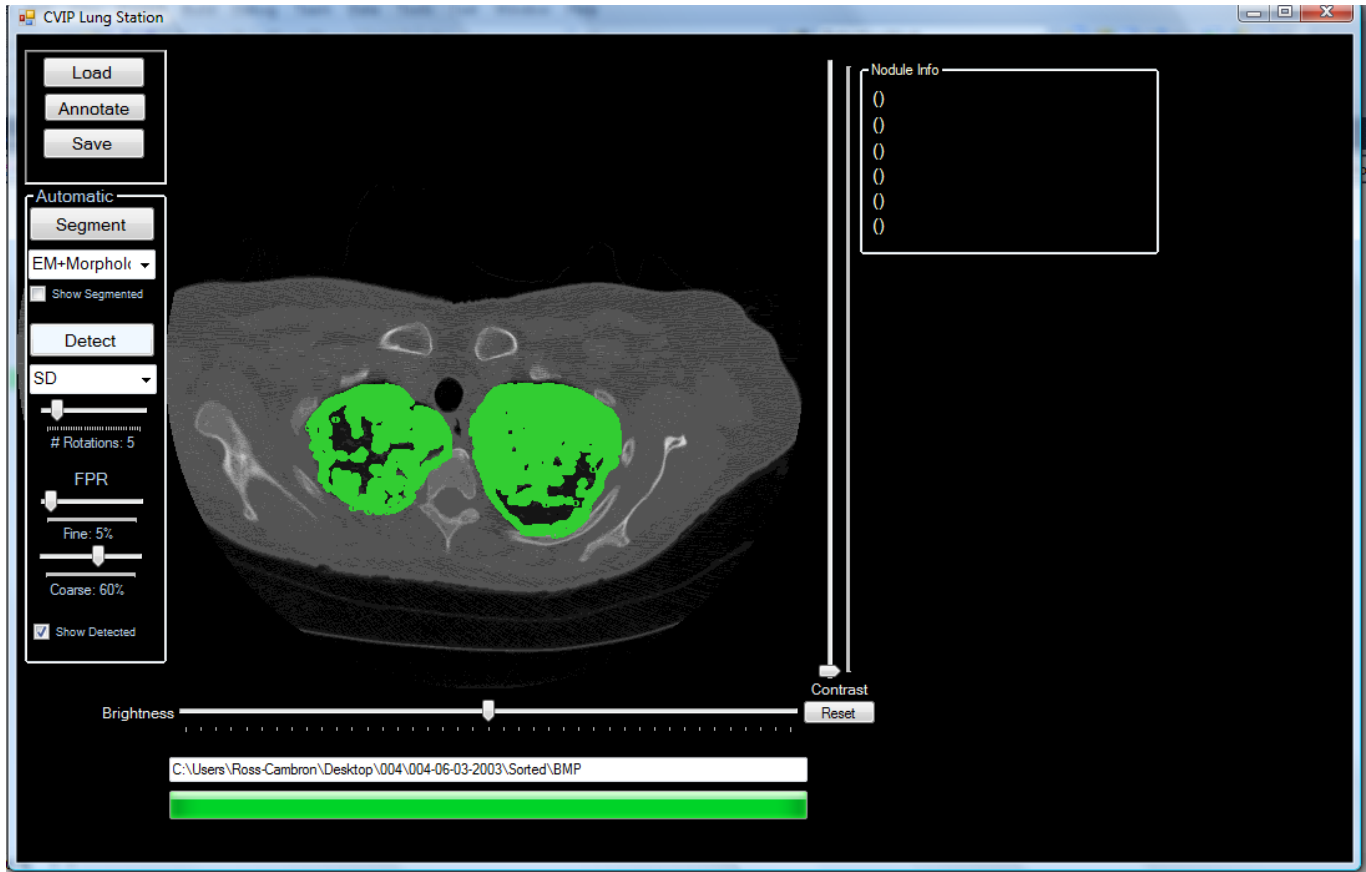

Figure 55: Nodule detection carried out using the SD with a Coarse FPR sensitivity set to 0.6 and a Fine FPR sensitivity set to $5 \%$. 


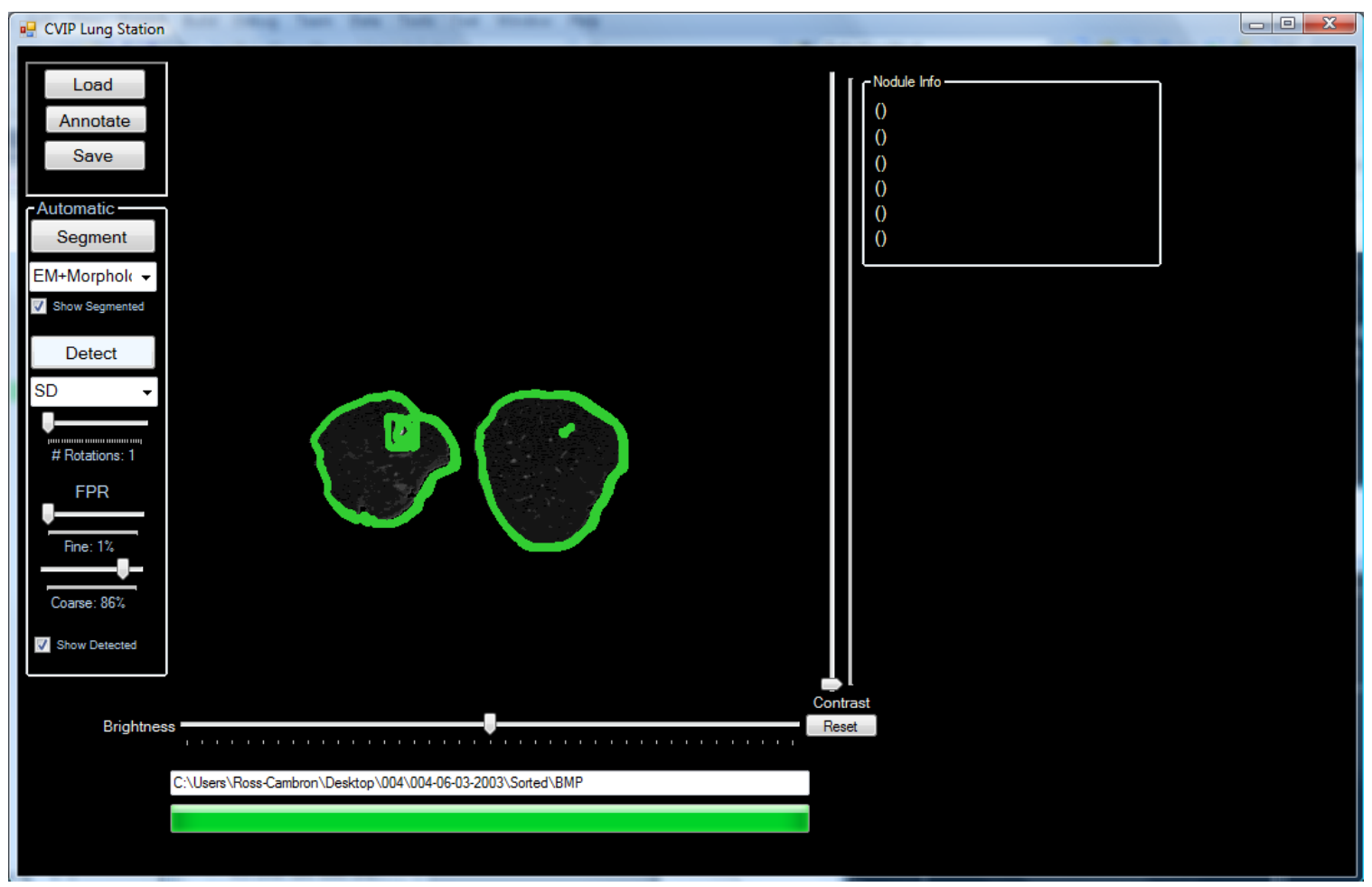

Figure 56 Nodule detection carried out using the SD with a Coarse FPR sensitivity set to 0.86 and a Fine FPR sensitivity set to $1 \%$. 